\title{
VARIATIONAL CONSTRUCTION OF ORBITS OF TWIST DIFFEOMORPHISMS
}

\author{
JOHN N. MATHER
}

\section{INTRODUCTION}

In this paper, we construct certain random or chaotic orbits in certain exact area-preserving diffeomorphisms of the cylinder. The mappings we consider are finite compositions of monotone twist diffeomorphisms, all of the same sign (i.e., either all positive monotone or negative monotone). For simplicity, we consider only finite compositions of positive monotone twist diffeomorphisms, since the case of finite compositions of negative monotone twist diffeomorphisms may be reduced to this case, by taking inverses. The class $\mathscr{P}^{1}$ of finite compositions of exact area-preserving positive monotone twist diffeomorphisms of the infinite cylinder is defined in $\S 1$.

Let $\bar{f} \in \mathscr{P}^{1}$. There is a closed $\bar{f}$-invariant subset $M_{\bar{f}}$ of the infinite cylinder, which has been much discussed in the literature, e.g., [3]. This set is defined by a variational principle: the orbits in it are minimal with respect to the action, in a sense that is defined in $\S 2$. Because orbits in $M_{\bar{f}}$ are those that minimize the action, the set $M_{\bar{f}}$ is intrinsically defined, although giving an intrinsic definition is somewhat involved. The relatively simple definition given in $\S 2$ is not intrinsic; we give an intrinsic definition in $\S 3$.

All $\bar{f}$-invariant simple closed curves that are not null-homotopic are in $M_{\bar{f}}$ (Proposition 2.8). In addition the Aubry-Mather sets are in $M_{\bar{f}}$, essentially by definition.

In $\S 4$, we define the notion of a Birkhoff region of instability $R$ for $\bar{f}$ and state the main results, Theorems 4.1 and 4.2. These results are self-explanatory, with the one additional remark that $M_{\bar{f}, \omega}$ denotes the set of orbits of $M_{\bar{f}}$ of (angular) rotation number $\omega$. Briefly, Theorem 4.1 shows the possibility of constructing an orbit with prescribed $\alpha$ - and $\omega$-limit sets, when these are both Aubry-Mather sets in the same Birkhoff region of instability, and Theorem 4.2 shows the possibility of constructing orbits that are "random" in the sense that they approach in turn each of a prescribed sequence of Aubry-Mather sets, provided that these all lie in the same Birkhoff region of instability.

Received by the editors April 4, 1990 and, in revised form, March 6, 1991.

1991 Mathematics Subject Classification. Primary 58F10, 58F11, $28 \mathrm{D} 05$.

The author was supported by NSF contract DMS-8806067.01 and a Guggenheim Fellowship. 
The proof of Theorem 4.1 (which is completed in $\S 11$ ) actually shows more than is stated in Theorem 4.1. Thus, suppose that $\mathscr{O}_{-}$and $\mathscr{O}_{+}$are minimal (i.e., action-minimizing) orbits in the same Birkhoff region of instability $R$. Let $\Gamma_{-}$and $\Gamma_{+}$be the lower and upper boundaries of $R$ and let $\rho\left(\Gamma_{-}\right)$and $\rho\left(\Gamma_{+}\right)$ be their (angular) rotation numbers. Let $\rho\left(\mathscr{O}_{-}\right)$and $\rho\left(\mathscr{O}_{+}\right)$be the (angular) rotation numbers of $\mathscr{O}_{-}$and $\mathscr{O}_{+}$. The statement of Theorem 4.1 suggests the following question: Does there exist an orbit $\mathscr{O}$ that is $\alpha$-asymptotic to $\mathscr{O}_{-}$ and $\omega$-asymptotic to $\mathscr{O}_{+}$? In $\S 11$, we show that there does exist such an orbit if the strict inequalities $\rho\left(\Gamma_{-}\right)<\rho\left(\mathscr{O}_{ \pm}\right)<\rho\left(\Gamma_{+}\right)$hold (for both of these orbits), and $\rho\left(\mathscr{O}_{-}\right)$and $\rho\left(\mathscr{O}_{+}\right)$are both irrational. If $\rho\left(\mathscr{O}_{-}\right)$or $\rho\left(\mathscr{O}_{+}\right)$is a rational number $p / q$ (but $\left.\rho\left(\Gamma_{-}\right)<\rho\left(\mathscr{O}_{ \pm}\right)<\rho\left(\Gamma_{+}\right)\right)$, this conclusion is still true under additional hypotheses, which are explained in $\S 11$. These additional hypotheses hold if $f$ is generic, e.g., if there is only one minimal periodic orbit of angular rotation number $p / q$. Of course, similar comments still hold if both $\rho\left(\mathscr{O}_{-}\right)$ and $\rho\left(\mathscr{O}_{+}\right)$are rational numbers.

The fact that we have to exclude the cases $\omega_{-}=\rho\left(\Gamma_{-}\right)$or $\rho\left(\Gamma_{+}\right)$when $\omega_{-}$is rational and the cases $\omega_{+}=\rho\left(\Gamma_{-}\right)$or $\rho\left(\bar{\Gamma}_{+}\right)$when $\omega_{+}$is rational in the statement of Theorem 4.1 is annoying. However, in these cases, it is still possible to construct orbits that approach the boundary. This, too, is discussed in $\S 11$.

Our discussion in $\S 11$ leaves open some problems. Most of these are complicated to formulate but easy to decide using the methods of this paper. For this reason, we do not bother to formulate them. However, there are two problems that seem interesting, important, and difficult. In both problems, we assume that $\rho_{-}$is irrational. Let $\mathscr{O}_{-}$be an orbit in the lower boundary of $R$.

Problem 1. Does there always exist an orbit $\mathscr{O}$ in $R$ that is $\alpha$-asymptotic to $\mathscr{O}_{-}$?

Problem 2. Does there ever exist an orbit $\mathscr{O}$ in $R$ that is $\alpha$-asymptotic to $\mathscr{O}_{-}$?

We could also ask for an orbit $\omega$-asymptotic to the given orbit in the boundary, or replace "lower boundary" and " $\rho_{-}$" with "upper boundary" and " $\rho_{+}$," but such changes obviously produce equivalent problems. We note, however, that if we drop the condition that $\rho_{-}$be irrational, the above problems have easy solutions.

We may vary this problem by asking the same questions with the restriction that $\bar{f}$ be $C^{r}$, for $r \geq 1$, or for $r=\infty$, or for $r=\omega$.

Theorems 4.1 and 4.2 assert the existence of orbits $\mathscr{O}$ with certain properties. We prove the existence of these orbits by variational means. The basic scheme of the proof is explained in $\S 5$. Let $\mathscr{J}=\left(\ldots, J_{i}, \ldots\right)$ be a bi-infinite sequence of closed, connected, nonempty subsets of $\mathbb{R}$. By Proposition 5.1 , if there exist arbitrarily small and arbitrarily large $i$ for which $J_{i}$ is bounded, then there exists a " $\mathscr{J}$-minimal configuration," i.e., a bi-infinite sequence $x=\left(\ldots, x_{i}, \ldots\right)$ of real numbers that is minimal for the variational principle $h$ associated to $f$ among such bi-infinite sequences that satisfy $x_{i} \in J_{i}$, for every $i$. We say 
$x$ is $\mathscr{J}$-free if $x_{i} \in$ int $J_{i}$, for every $i$. By Proposition 5.2, every $\mathscr{J}$-free and $\mathscr{J}$-minimal configuration $x=\left(\ldots, x_{i}, \ldots\right)$ corresponds to an $f$-orbit $\left(\ldots,\left(x_{i}, y_{i}\right), \ldots\right)$. Both of these propositions are easy to prove. The main part of the proof consists of finding conditions on $\mathcal{J}$ that imply that every $\mathscr{J}$-minimal configuration is $\mathscr{J}$-free. (Usually, there is only one $\mathscr{J}$-minimal configuration.) Then we find further conditions on $\mathscr{J}$ that imply that the orbit that corresponds to a $\mathscr{J}$-minimal and $\mathscr{J}$-free configuration has the properties required by Proposition 4.1 or Proposition 4.2.

$0.5 \mathrm{pc}$

The conditions that imply that a $\mathscr{J}$-minimal configuration is $\mathscr{J}$-free are broken down into various conditions of the following form: it is assumed that $J_{i}$ has a certain form for $j_{0}-K_{0} \leq i \leq j_{1}+K_{1}$ and then it is proved that if $\xi=\left(\ldots, \xi_{i}, \ldots\right)$ is $\mathscr{J}$-minimal, then $\xi_{i} \in$ int $J_{i}$, for $j_{0} \leq i \leq j_{1}$. Such a $\xi$ may be said to be "partially $\mathcal{J}$-free." The first of these results is Proposition 6.1. Here, $J_{i}$ is defined for $j_{0}-K_{0} \leq i \leq j_{1}+K_{1}$ in terms of a minimal configuration $x=\left(\ldots, x_{i}, \ldots\right)$. We let $\omega$ be the (angular) rotation number of $x$ and suppose that there exists $a \in \mathbb{R}$ such that Peierls's barrier $P_{\omega}(a)$ is positive. (We recall the definition of $P_{\omega}(a)$ at the beginning of $\S 6$ : it is a nonnegative function of $a \in \mathbb{R}$ that satisfies a Lipschitz condition as a function of $a \in \mathbb{R}$, is periodic of period 1 , and, in the case $\omega$ is irrational, vanishes identically on $\mathbb{R}$ if and only if there exists an invariant circle for $\bar{f}$ that winds around the cylinder and has angular rotation number $\omega$.) In the case of Proposition 6.1, we take $K_{0}=K_{1}=K(\omega, a)$, where the latter number is defined in $\S 6$. (In some cases, $K(\omega, a)$ is undefined. Proposition 6.1 applies only when $K(\omega, a)$ is defined.) Actually, $K(\omega, a)$ depends on $f$ as well as on $\omega$ and $a$, but we suppress $f$ from the notation, since $f$ is held fixed throughout the proofs of Theorems 4.1 and 4.2. More specifically, $K(\omega, a)$ depends only on $\omega$ and $\theta / P_{\omega}(a)$, where $\theta=\cot \beta$, and the angle $\beta$ is a lower bound for the amount of twisting of the diffeomorphisms $\bar{f}_{i}$ of which $\bar{f}$ is the composition. Note that $K(\omega, a)$ is defined when $\omega$ is irrational and $P_{\omega}(a)>0$. If $\omega=p / q$ in lowest form, then $K(\omega, a)$ is defined if and only if $q-1>2 \theta / P_{\omega}(a)$.

In Proposition 6.1, $J_{i}$ is taken to be a closed interval of length 1 for $j_{0}$ $K(\omega, a) \leq i \leq j_{1}+K(\omega, a)$. Specifically, $J_{i}=\left[a_{i}, a_{i}+1\right]$, where $a_{i}$ is defined by the conditions $a_{i}-a \in \mathbb{Z}$ and $x_{i} \in\left(a_{i}, a_{i}+1\right)$. Because of the periodicity of Peierls's barrier, we have $P_{\omega}\left(a_{i}\right)=P_{\omega}(a)>0$, and it is this positivity that permits us to prove that $\xi_{i} \in\left(a_{i}, a_{i}+1\right)$ for $j_{0} \leq i \leq j_{1}$.

The construction in Proposition 6.1 is very similar to the basic construction in [15], which, however, was used for a different purpose.

The quantity $K(\omega, a)$ is analyzed more thoroughly in $\S 7$, where a closely related quantity $K(\omega, \varepsilon)$ is introduced. This is a function of $\omega \in \mathbb{R}$ and $\varepsilon>0$. It is undefined if $\omega$ is a rational number, $p / q$ in lowest terms, with $q \varepsilon \leq 1$. In all other cases, it is a positive integer. Note that $K(\omega, \varepsilon)$ depends only on $\omega$ and $\varepsilon$ : it is a purely number-theoretic function, which has nothing to do with dynamics. It is related to $K(\omega, a)$ in the following way: Let $k$ be the smallest 
integer $>2 \theta / P_{\omega}(a)$. Then $K(\omega, a)=K\left(\omega, k^{-1}\right)$, by definition. The most important property of $K(\omega, \varepsilon)$, aside from its relation to $K(\omega, a)$, is the fact (proved in $\S 7$ ) that $K(\omega, \varepsilon)$ is bounded for $\omega \in \Omega$, if $\Omega$ is a closed interval that does not contain any rational numbers whose denominators are $\leq \varepsilon^{-1}$, and $K(\omega, \varepsilon) \rightarrow+\infty$ as $\omega$ approaches a rational number whose denominator is $\leq \varepsilon^{-1}$. This follows from the estimate $1 / 2 \Delta_{\varepsilon}(\omega) \leq K(\omega, \varepsilon) \leq 2 / \Delta_{\varepsilon}(\omega)$ stated in $\S 7$. Using this and Proposition 6.1, we are able, in $\S 7$, to prove Propositions 7.4 and 7.5, whose statements are self-explanatory. These propositions show how far, using Proposition 6.1, we are able to get towards proving Theorems 4.1 and 4.2 .

There are two limitations on using Proposition 6.1. The first comes from the fact that to use Proposition 6.1, we must fix $a$, once and for all. We wish to be able to move freely in a Birkhoff region of instability $R$ and the only condition we have on $R$ is that if $\Gamma$ is an invariant closed curve in $R$, noncontractible to a point in $R$, then $\Gamma$ is one of the boundary curves, $\Gamma_{-}$or $\Gamma_{+}$. From this, it follows that if $\rho\left(\Gamma_{-}\right)<\omega<\rho\left(\Gamma_{+}\right)$, then there exists $a$ such that $P_{\omega}(a)>0$. However, it may not be the case that the same $a$ works for all $\omega$. This difficulty is easily overcome, by Proposition 6.2. Here, the moduli of continuity for $P_{\omega}$ [17, Theorem 2.2] are used. These are the inequalities $\left|P_{p / q+}(a)-P_{\omega}(a)\right| \leq$ $C \theta|\omega q-p|$, in the case $\omega>p / q$, and $\left|P_{p / q-}(a)-P_{\omega}(a)\right| \leq C \theta|\omega q-p|$, in the case $\omega<p / q$. These inequalities imply that $P_{\omega}(a)$, as a function of $\omega$, is continuous at irrationals, and consequently if $P_{\omega}(a)>0$ for some irrational number $\omega=\omega_{0}$, the same holds for all $\omega$ sufficiently near $\omega_{0}$.

The more serious limitation on the use of Proposition 6.1 arises from its inapplicability when $\omega=p / q$ and $q$ is too small. Specifically, it does not apply when $K(\omega, a)$ is undefined, i.e., when $q-1 \leq 2 \theta / P_{\omega}(a)$. To get past rational rotation numbers with small denominator, a different method is needed. This is provided by Proposition 8.1. The case of rational rotation number $p / q$ for $f$ may be reduced to the case of zero rotation number for $F$ defined by $F(x, y)=f^{q}(x, y)-(p, 0)$. The latter has a variational principle $H$, which is defined in terms of the variational principle $h$ of $f$ by means of the conjunction operation, introduced in [16]. Proposition 8.1, which explains how to get past rational rotation numbers, is stated in terms of $F$ and $H$ and zero rotation number for (relative) simplicity. The proof in $\S 8$ is the heart of this paper and the part that is conceptually new vis-à-vis [15]. Then in Lemma 9.3, we apply the results obtained for $F$ and $H$ in Proposition 8.1 to $f$ and $h$. The $J_{i}$ of Proposition 8.1 permit us to define closed, connected, nonempty subsets $J_{\alpha}^{*}$ of $\mathbb{R}$ that do the trick in Lemma 9.3. The proof of Lemma 9.3, although long, is simply a matter of checking that obvious conditions are satisfied.

In $\S 10$, we give conditions on $\mathscr{J}=\left(\ldots, J_{i}, \ldots\right)$ that guarantee that a $\mathscr{J}$ minimal and $\mathscr{J}$-free configuration $\xi=\left(\ldots, \xi_{i}, \ldots\right)$ has the property that the $f$-orbit corresponding to it approaches $M_{f, \omega}$ as closely as is required in Theorems 4.1 and 4.2. 
In $\S 11$, we conclude the proofs of Theorems 4.1 and 4.2 and draw some further conclusions from these proofs.

\section{FINITE COMPOSITIONS OF EXACT AREA-PRESERVING POSITIVE MONOTONE TWIST DIFFEOMORPHISMS OF THE INFINITE CYLINDER AND THEIR VARIATIONAL PRINCIPLES}

In the next section, we review results concerning minimal orbits. These results have been obtained only for a special class of mappings, whose definition is somewhat complicated. In this section, we recall the definition of this special class of mappings and the notion of the variational principle associated to a mapping in this class.

Let $\beta>0$. We denote by $\mathscr{C}_{\beta}^{1}$ the set of $C^{1}$ exact area-preserving, orientation-preserving, positive monotone twist diffeomorphisms of the infinite cylinder that preserve the ends, twist each end infinitely, and have $\beta$ as a uniform lower bound for the amount of twisting. A detailed definition of $\mathscr{C}_{\beta}^{1}$ is given in $[16, \S 2]$, where it is called $J_{\beta}$.

For the convenience of the reader, we repeat the definition of $\mathscr{C}_{\beta}^{1}$ here. By the infinite cylinder, we mean $(\mathbb{R} / \mathbb{Z}) \times \mathbb{R}$. We let $\theta(\bmod 1)$ denote the standard coordinate of $\mathbb{R} / \mathbb{Z}$ and let $y$ denote the standard coordinate of $\mathbb{R}$. Consider a $C^{1}$ diffeomorphism $\bar{f}$ of the infinite cylinder into itself. We will write $\bar{f}(\theta, y)=\left(\theta^{\prime}, y^{\prime}\right)$, so the pull-back of the 1 -form $y^{\prime} d \theta^{\prime}$ by $\bar{f}$ is $y d \theta$. Note that the condition that $\bar{f}$ be area-preserving and orientation-preserving is $d y^{\prime} \wedge d \theta^{\prime}=d y \wedge d \theta$. In other words, $y^{\prime} d \theta^{\prime}-y d \theta$ should be closed. We will require the stronger condition that $y^{\prime} d \theta^{\prime}-y d \theta$ be exact. When $\bar{f}$ satisfies this condition, we say that it is exact area-preserving and orientation-preserving.

The positive monotone twist condition is that $\partial \theta^{\prime} / \partial y>0$ (where we take $\theta$ and $y$ as the independent variables). Geometrically, this means that $\bar{f}$ maps each vertical line in the infinite cylinder into a curve that always twists around the infinite cylinder in the positive direction. The positive monotone twist condition is equivalent to the condition that $\partial \theta / \partial y^{\prime}<0$ (where we take $\theta^{\prime}$ and $y^{\prime}$ to be the independent variables). The condition that $\beta$ is a uniform lower bound for the amount of twisting means that $\partial \theta^{\prime} / \partial y>\tan \beta$ and $\partial \theta / \partial y^{\prime}<$ $-\tan \beta$; geometrically, this means that the image under $\bar{f}$ of a vertical line always makes an angle greater than $\beta$ with every vertical line, and likewise for the image of a vertical line under $\bar{f}^{-1}$.

The condition that $\bar{f}$ preserve the ends of the infinite cylinder is evidently that $y^{\prime} \rightarrow \pm \infty$ as $y \rightarrow \pm \infty$.

We will denote the coordinates of the universal cover $\mathbb{R}^{2}$ of the infinite cylinder by $x$ and $y$, so that $\theta \equiv x(\bmod 1)$. We let $f$ be a lift of $\bar{f}$ to the universal cover, and set $f(x, y)=\left(x^{\prime}, y^{\prime}\right)$. The condition that $f$ twist each end infinitely means that $x^{\prime} \rightarrow \pm \infty$ as $y \rightarrow \pm \infty$ (where $x$ is held fixed). Equivalently, $x \rightarrow \mp \infty$ as $y^{\prime} \rightarrow \pm \infty$ (where $x^{\prime}$ is held fixed). 
This completes the list of conditions for $\bar{f}$ to be a member of $\mathscr{C}_{\beta}^{1}$. We let $\mathscr{C}^{1}=\bigcup_{\beta>0} \mathscr{C}_{\beta}^{1}$.

Let $\bar{f} \in \mathscr{C}^{1}$ and let $f$ be a lift of $\bar{f}$ to the universal cover $\mathbb{R}^{2}$. As above, we set $f(x, y)=\left(x^{\prime}, y^{\prime}\right)$. The conditions we have imposed imply that graph $f \subset \mathbb{R}^{2} \times \mathbb{R}^{2}$ projects diffeomorphically onto the $\left(x, x^{\prime}\right)$-plane. Thus, we may take $x$ and $x^{\prime}$ as the independent variables and write $\left(y^{\prime} d x^{\prime}-y d x\right) \mid$ graph $f=$ $d h$, where $h=h\left(x, x^{\prime}\right)$ is a $C^{2}$ function on $\mathbb{R}^{2}$. In classical mechanics, $h$ is called a generating function of $f$. It is characterized by

$$
f(x, y)=\left(x^{\prime}, y^{\prime}\right) \Leftrightarrow y=-\partial_{1} h\left(x, x^{\prime}\right) \text { and } y^{\prime}=\partial_{2} h\left(x, x^{\prime}\right),
$$

and it is uniquely defined up to addition of a constant.

Such a generating function $h$ satisfies several useful conditions:

$$
\begin{gathered}
h\left(x+1, x^{\prime}+1\right)=h\left(x, x^{\prime}\right), \quad \text { for all } x, x^{\prime} \in \mathbb{R} . \\
\lim _{|\xi| \rightarrow \infty} h(x, x+\xi)=\infty, \quad \text { uniformly in } x .
\end{gathered}
$$

$\left(\mathrm{H}_{5}\right) \quad$ There exists a positive continuous function $\rho$ on $\mathbb{R}^{2}$ such that

$$
h\left(\xi, x^{\prime}\right)+h\left(x, \xi^{\prime}\right)-h\left(x, x^{\prime}\right)-h\left(\xi, \xi^{\prime}\right) \geq \int_{x}^{\xi} \int_{x^{\prime}}^{\xi^{\prime}} \rho,
$$

if $x<\xi$, and $x^{\prime}<\xi^{\prime}$.

Moreover, if $\bar{f} \in \mathscr{C}^{1}$ and $\theta=\cot \beta$, then $h$ satisfies

$\left(\mathrm{H}_{6 \theta}\right) \quad x \mapsto \theta x^{2} / 2-h\left(x, x^{\prime}\right)$ is convex, for any $x^{\prime}$, and $x^{\prime} \mapsto \theta x^{\prime 2} / 2-$ $h\left(x, x^{\prime}\right)$ is convex, for any $x$.

We recall that a function of one variable is convex if the line segment joining two points of its graph lies on or above the graph.

The fact that $h$ satisfies these conditions was proved in [16]. See the example in $[16, \S 3]$ and the discussion in [16, $\S 4]$. (Here, we retain the notation of [16]. There, we also discussed further conditions $\mathrm{H}_{3}$ and $\mathrm{H}_{4}$, introduced by Bangert [3]. The conditions $\mathrm{H}_{3}$ and $\mathrm{H}_{4}$ will not be needed in this paper.)

Our reason for considering $h$ is that it allows us to define a $\bar{f}$-invariant closed subset $M_{\bar{f}}$ of the infinite cylinder whose properties appear to be quite important in the study of the dynamics of $\bar{f}$. This set is the union of all minimal orbits, in the terminology of Bangert [3], which was adopted in Mather [16-18]; previously these had been called "minimal energy orbits" in Aubry and Le Daeron [2] and Mather [13]. In fact, it is possible to define $M_{\bar{f}}$ not only when $\bar{f} \in \mathscr{C}^{1}$, but also when $\bar{f}$ is a finite composition of elements of $\mathscr{C}^{1}$.

We will postpone defining $M_{\bar{f}}$ until the next section and first define $h$ in the more general situation when $\bar{f}=\bar{f}_{k} \circ \cdots \circ \bar{f}_{1}$, where $\bar{f}_{i} \in \mathscr{C}^{1}$. This is based on the notion of conjunction, introduced in $[16, \S 5]$. Let $h_{1}, h_{2}$ be two real-valued continuous functions on $\mathbb{R}^{2}$ that satisfy $\left(\mathrm{H}_{2}\right)$. We set

$$
h_{1} * h_{2}\left(x, x^{\prime}\right)=\min _{\xi}\left(h_{1}(x, \xi)+h_{2}\left(\xi, x^{\prime}\right)\right) \text {. }
$$


It is easily verified that $h_{1} * h_{2}$ is defined, continuous, and satisfies $\left(\mathrm{H}_{2}\right)$. In $[16, \S 5]$, we dubbed this operation conjunction. Thus, the class of continuous, real-valued functions on $\mathbb{R}^{2}$ that satisfy $\left(\mathrm{H}_{2}\right)$ is closed under the operation of conjunction. Conjunction is clearly associative.

We will denote by $\mathscr{P}_{\beta}^{1}$ the set of all $C^{1}$ diffeomorphisms of the infinite cylinder that may be expressed as finite compositions of elements of $\mathscr{C}_{\beta}^{1}$. We set $\mathscr{P}^{1}=\bigcup_{\beta>0} \mathscr{P}_{\beta}^{1}$.

For $\bar{f} \in \mathscr{P}^{1}$, we consider a decomposition $\bar{f}=\bar{f}_{k} \circ \cdots \circ \bar{f}_{1}$ with $\bar{f}_{i} \in \mathscr{C}^{1}$, let $f_{i}$ be a lift of $\bar{f}_{i}$ to the universal cover and set $h=h_{1} * \cdots * h_{k}$, where $h_{i}$ is a generating function of $f$. We call $h$ a variational principle associated to $f=f_{k} \circ \cdots \circ f_{1}$. In fact, except for addition of a constant, $h$ depends only on $f$ (and the coordinatization of the infinite cylinder), but we will not need this fact. (The proof is lengthy.) When $\bar{f} \notin \mathscr{C}^{1}, h$ is no longer a generating function, but it retains enough properties to permit the definition of $M_{\bar{f}}$.

In [16], we proved that a variational principle $h$ associated to a lift $f$ of $\bar{f} \in \mathscr{P}_{\beta}^{1}$ satisfies $\left(\mathrm{H}_{1}\right),\left(\mathrm{H}_{2}\right),\left(\mathrm{H}_{5}\right)$, and $\left(\mathrm{H}_{6 \theta}\right)$, with $\theta=\cot \beta$. The main step in the proof is [16, Lemma 5.3], which asserts that if $h_{1}$ and $h_{2}$ are continuous real-valued functions on $\mathbb{R}^{2}$ that satisfy these conditions, then so is $h_{1} * h_{2}$. (To see that [16, Lemma 5.3] applies, note that the conditions $\left(\mathrm{H}_{3}\right)$ and $\left(\mathrm{H}_{4}\right)$ follow from these conditions: $\left(\mathrm{H}_{3}\right)$ is a weaker form of $\left(\mathrm{H}_{5}\right)$ and we proved at the end of $[16, \S 4]$ that $\left(\mathrm{H}_{4}\right)$ follows from $\left(\mathrm{H}_{5}\right)$ and $\left(\mathrm{H}_{6}\right)$.) Lemma 5.3 of [16] is enough in view of the fact (which we quoted above from [16]) that the generating function of a lift $f$ of $\bar{f} \in \mathscr{C}_{\beta}^{1}$ satisfies $\left(\mathrm{H}_{1}\right),\left(\mathrm{H}_{2}\right),\left(\mathrm{H}_{5}\right)$, and $\left(\mathrm{H}_{6 \theta}\right)$. (Following the terminology of [16], we say that $h$ satisfies $\left(\mathrm{H}_{6}\right)$ if it satisfies $\left(\mathrm{H}_{6 \theta}\right)$ for some $\theta>0$.)

To simplify the notation, we will introduce the following definitions: Let $\theta>0$. We will say that a function $h: \mathbb{R}^{2} \rightarrow \mathbb{R}$ satisfies $\left(\mathrm{H}_{\theta}\right)$ if it is continuous and satisfies $\left(\mathrm{H}_{1}\right),\left(\mathrm{H}_{2}\right),\left(\mathrm{H}_{5}\right)$, and $\left(\mathrm{H}_{6 \theta}\right)$. As before, this condition depends on the number $\theta>0$, which is chosen in advance. We will also say that $h$ satisfies $(\mathrm{H})$ if it satisfies $\left(\mathrm{H}_{\theta}\right)$ for some $\theta>0$. To summarize this section, we have recalled the definition of the class $\mathscr{P}^{1}$ of finite compositions of exact areapreserving positive monotone twist diffeomorphisms of the infinite cylinder. We have associated a variational principle $h$ to any $\bar{f} \in \mathscr{P}^{1}$. We have recalled from [16] the fact that such an $h$ satisfies $(\mathrm{H})$.

\section{Minimal ORBITS}

In this section, we recall the definition of minimal orbit of a diffeomorphism $\bar{f} \in \mathscr{P}^{1}$. This is defined by means of the variational principle $h: \mathbb{R}^{2} \rightarrow \mathbb{R}$ associated to $\bar{f}$.

One extends $h$ to finite sequences of real numbers $\left(x_{j}, \ldots, x_{k}\right), j<k$, by 
setting

$$
h\left(x_{j}, \ldots, x_{k}\right)=\sum_{i=j}^{k-1} h\left(x_{i}, x_{i+1}\right) .
$$

One says that $\left(x_{j}, \ldots, x_{k}\right)$ is minimal with respect to $h$ if

$$
h\left(x_{j}, \ldots, x_{k}\right) \leq h\left(x_{j}^{*}, \ldots, x_{k}^{*}\right),
$$

for all finite sequences of real numbers $\left(x_{j}^{*}, \ldots, x_{k}^{*}\right)$ with $x_{j}=x_{j}^{*}$ and $x_{k}=$ $x_{k}^{*}$. One says that a bi-infinite sequence $\left(\ldots, x_{i}, \ldots\right)$ is minimal with respect to $h$ if for every $j<k$, the corresponding segment $\left(x_{j}, \ldots, x_{k}\right)$ of it is minimal (with respect to $h$ ).

This notion was introduced by Aubry and Le Daeron [1]. We were interested in it because it provided alternative proofs of results concerning the dynamics of area-preserving diffeomorphisms we obtained [12] and also led to other results. These results form the starting point of this paper, and we now recall them.

In the next to the last paragraph in $[16, \S 4]$, we showed that if $h$ satisfies $(\mathbf{H})$ and $\left(x_{j}, \ldots, x_{k}\right)$ is minimal with respect to $h$, then $\left(x_{j}, \ldots, x_{k}\right)$ is stationary with respect to $h$ in the sense that for $j<i<k$, the first partial derivatives $\partial_{2} h\left(x_{i-1} x_{i}\right)$ and $\partial_{1} h\left(x_{i}, x_{i+1}\right)$ both exist and

$$
\partial_{2} h\left(x_{i-1}, x_{i}\right)+\partial_{1} h\left(x_{i}, x_{i+1}\right)=0 \text {. }
$$

Of course, if $h$ were differentiable, this assertion would be trivial. However, $(\mathrm{H})$ does not imply differentiability of $h$. Moreover, in general, a variational principle $h$ associated to $\bar{f} \in \mathscr{P}^{1}$ need not be differentiable. Nonetheless, the proof that minimality implies stationarity if $h$ satisfies $(H)$ is very easy: $\left(\mathbf{H}_{6}\right)$ implies that the one-sided partial derivatives of $h$ exist and satisfy

$$
\partial_{1} h\left(x-, x^{\prime}\right) \geq \partial_{1} h\left(x+, x^{\prime}\right), \quad \partial_{2} h\left(x, x^{\prime}-\right) \geq \partial_{2} h\left(x, x^{\prime}+\right) .
$$

Minimality implies stationarity may then be proved in the same way it is proved when $h$ is differentiable. For more detail, we refer to [16, §4].

Let $\bar{f} \in \mathscr{P}^{1}$ and let $h$ be a variational principle associated to a lift $f$ of $\bar{f}$ to the universal cover $\mathbb{R}^{2}$. Consider a minimal configuration $\left(\ldots, x_{i}, \ldots\right)$ for $h$. Let

$$
y_{i}=-\partial_{1} h\left(x_{i}, x_{i+1}\right)=\partial_{2} h\left(x_{i-1}, x_{i}\right) .
$$

In the example in $[16, \S 5]$, we proved that $f\left(x_{i}, y_{i}\right)=\left(x_{i+1}, y_{i+1}\right)$, that is, $\left(\ldots,\left(x_{i}, y_{i}\right), \ldots\right)$ is an orbit of $f$.

To summarize, we proved (in the example in $[16, \S 5]$ ):

Proposition 2.1. Let $\bar{f} \in \mathscr{P}_{\beta}^{1}$. Let $f: \mathbb{R}^{2} \rightarrow \mathbb{R}^{2}$ be a lift of $\bar{f}$. Let $h$ be a variational principle associated to $f$. Then $h$ satisfies $\left(\mathrm{H}_{\theta}\right)$, with $\theta=\cot \beta$. Consider a bi-infinite sequence $x=\left(\ldots, x_{i}, \ldots\right) \in \mathbb{R}^{\mathbb{Z}}$ of real numbers and suppose that $x$ is minimal (with respect to $h$ ). Then there exists

$$
y_{i}=-\partial_{1} h\left(x_{i}, x_{i+1}\right)=\partial_{2} h\left(x_{i+1}, x_{i}\right)
$$

for $i \in \mathbb{Z}$ and $\left(\ldots,\left(x_{i}, y_{i}\right), \ldots\right)$ is an orbit of $f$, i.e., $f\left(x_{i}, y_{i}\right)=\left(x_{i+1}, y_{i+1}\right)$. 
Thus, to each minimal (with respect to $h$ ) $x \in \mathbb{R}^{\mathbb{Z}}$, one associates an orbit of $f$. This association is obviously injective. One calls the orbits of $f$ obtained in this way minimal orbits. An orbit of $f$ lies in $\mathbb{R}^{2}$; if we project it on $(\mathbb{R} / \mathbb{Z}) \times \mathbb{R}$, we obtain an orbit of $\bar{f}$. If the orbit of $f$ is minimal, one says that the corresponding orbit of $\bar{f}$ is minimal. We let $M_{f} \subset \mathbb{R}^{2}$ denote the union of all minimal orbits of $f$ and $M_{\bar{f}} \subset(\mathbb{R} / \mathbb{Z}) \times \mathbb{R}$ the union of all minimal orbits of $\bar{f}$.

Next, in Propositions 2.2-2.4, we list several properties of minimal configurations, due to Aubry and Le Daeron [1] and generalized by Bangert [3]. For the proofs, we refer to the latter paper, which assumes that the "variational principle" $h$ satisfies four hypotheses, which Bangert labels $\left(\mathrm{H}_{1}\right)-\left(\mathrm{H}_{4}\right)$. To apply Bangert's results, we need only remark that our condition $(\mathrm{H})$ implies Bangert's conditions $\left(\mathrm{H}_{1}\right)-\left(\mathrm{H}_{4}\right)$. Indeed, in the last paragraph of [16, §4], we showed that $\left(\mathrm{H}_{5}\right)$ and $\left(\mathrm{H}_{6}\right)$ imply $\left(\mathrm{H}_{3}\right)$ and $\left(\mathrm{H}_{4}\right)$.

Thus, we may apply the results of Bangert [3] to any $h$ that satisfies condition $(\mathrm{H})$; by Proposition 2.1 such results give information about orbits of $\bar{f}$ that lie in $M_{\bar{f}}$. These results give a fairly complete picture of the dynamics of $\bar{f} \mid M_{\bar{f}}$.

We will call elements of $\mathbb{R}^{\mathbb{Z}}$ configurations. If $x$ and $x^{*}$ are any two configurations, we will say that $x<x^{*}$ if $x_{i}<x_{i}^{*}$, for all $i$. We will say that $x$ and $x^{*}$ are comparable if $x<x^{*}, x=x^{*}$, or $x>x^{*}$.

If $x$ is a configuration, and $p, q \in \mathbb{Z}$, we let $T_{p, q} x$ denote the configuration defined by

$$
\left(T_{p, q} x\right)_{i}=x_{i+q}-p .
$$

We call the various configurations $T_{p, q} x$ translates of $x$. Note that if $x$ is a minimal configuration with respect to $h$ (where $h$ satisfies $(\mathrm{H})$ ), then all the translates of $x$ are also minimal (with respect to $h$ ). This is an immediate consequence of $\left(\mathrm{H}_{1}\right)$.

Proposition 2.2. If $h$ satisfies $(\mathrm{H})$, and $x$ is any minimal configuration with respect to $h$, then any two translates of $h$ are comparable.

Proof. See Bangert [3, Theorem 3.13].

If $T_{p, q} x>x$, then $T_{l p, l q} x>x$ for every positive integer $l$, since $T_{l p, l q}=$ $T_{p, q}^{l}$ (iteration of $T_{p, q} l$ times). Consequently, if we consider only $q>0$, then whether or not $T_{p, q} x>x$ or $<x$ depends only on $p / q$. Let $\rho_{-}(x)$ denote the set of $p / q$ for which $T_{p, q} x<x$ (when $q>0$ ) and $\rho_{+}(x)$ denote the set of $p / q$ for which $T_{p, q} x>x$.

Proposition 2.3. If $h$ satisfies $(\mathrm{H})$, and $x$ is any minimal configuration with respect to $h$, then $\left(\rho_{-}(x), \rho_{+}(x)\right)$ is a Dedekind cut of the set of rational numbers, i.e., every element of $\rho_{-}(x)$ is less than every element of $\rho_{+}(x)$, neither $\rho_{-}(x)$ nor $\rho_{+}(x)$ is empty, and $\rho_{-}(x) \cup \rho_{+}(x)$ contains all but at most one rational number. 
Proof. It is easy to see that Proposition 2.2 implies all but the statement that neither $\rho_{-}(x)$ nor $\rho_{+}(x)$ is empty. This statement follows from [3, Corollary 3.16].

We set $\tilde{\rho}(x)=\left(\rho_{-}(x), \rho_{+}(x)\right)$ and call $\tilde{\rho}(x)$ the rotation symbol of $x$. We let $\rho(x)$ denote the cut point of $\widetilde{\rho}(x)$, i.e., the unique real number such that every element of $\rho_{-}(x)$ is $\leq \rho(x)$ and every element of $\rho_{+}(x)$ is $\geq \rho(x)$. The number $\rho(x)$ is called the rotation number of $x$.

We let $\mathscr{S}$ (the symbol space) denote the set of Dedekind cuts of the rational numbers. We provide $\mathscr{S}$ with its standard order: if $(A, B)$ and $\left(A^{\prime}, B^{\prime}\right)$ are Dedekind cuts, $(A, B) \leq\left(A^{\prime}, B^{\prime}\right)$ means $A \subset A^{\prime}$ and $B^{\prime} \subset B$. The canonical mapping $\mathscr{S} \rightarrow \mathbb{R}$ that associates to a Dedekind cut its cut point is order-preserving.

If $\omega$ is an irrational number, there is just one Dedekind cut of the rational numbers that has it as cut point. We denote this Dedekind cut also by $\omega$. For a rational number $p / q$, there are three Dedekind cuts $(A, B)$ that have it as cut point, depending on whether $p / q$ is in $A, B$, or neither. We denote these Dedekind cuts by $p / q+, p / q-$, and $p / q$. Thus, $p / q-<p / q<p / q+$. In this way, we identify the set $\mathbb{R}$ of real numbers with a subset of the symbol space $\mathscr{S}$. In other words, if $\omega \in \mathbb{R}$, we identify it with the Dedekind cut $(A, B)$, where $A$ consists of all numbers less than $\omega$ and $B$ consists of all numbers greater than $\omega$.

Since $\widetilde{\rho}(x) \in \mathscr{S}$, this notation means that we think of $\widetilde{\rho}(x)$ as being an irrational number, or having one of the forms $p / q-, p / q$, or $p / q+$, where $p / q$ is a rational number.

Proposition 2.4. Let $h$ satisfy $(\mathrm{H})$ and let $\omega \in \mathbb{R}$. Then there exists a minimal configuration $x$ (with respect to $h$ ) that satisfies $\rho(x)=\omega$.

Proof. See Bangert [3, Theorem 3.17].

Our interest in these results lies in their application to the structure of $M_{\bar{f}}$, where $\bar{f} \in \mathscr{P}^{1}$. It follows easily from the definitions that $M_{\bar{f}}$ is a closed $\bar{f}$-invariant set of the infinite cylinder.

If $\mathscr{O}=\left(\ldots,\left(x_{i}, y_{i}\right), \ldots\right)$ is an orbit of a lift $f$ of $\bar{f}$ to the universal cover, then the rotation number of $\mathscr{O}$ is defined as

$$
\rho(\mathscr{O})=\lim _{i \rightarrow \pm \infty} x_{i} / i
$$

if this limit exists. We will use the same terminology for orbits of $\bar{f}$ : if $\overline{\mathscr{O}}$ is the projection of $\mathscr{O}$ on the infinite cylinder, we define its rotation number to be $\rho(\widetilde{\mathscr{O}})=\rho(\mathscr{O})$. There is ambiguity here: $\rho(\overline{\mathscr{O}})$ depends on the choice of lift $f$; if we change the lift, the rotation number changes by an integer. However, the change is the same for all orbits, and we will ignore this ambiguity, assuming that the lift $f$ of $\bar{f}$ is fixed throughout the discussion. 
Let $\bar{f} \in \mathscr{P}^{1}$, let $f$ be a lift of it, and let $h$ be a variational principle associated to $f$. Let $x=\left(\ldots, x_{i}, \ldots\right)$ be a minimal configuration, with respect to $h$. By Proposition 2.1 , there is an orbit $\mathscr{O}=\left(\ldots,\left(x_{i}, y_{i}\right), \ldots\right)$ in $M_{f}$ associated to $\left(\ldots, x_{i}, \ldots\right)$; plainly, we have

$$
\rho(\mathscr{O})=\rho(x) .
$$

Thus, Propositions 2.3 and 2.4 imply

Proposition 2.5. Every $\bar{f}$-orbit in $M_{\bar{f}}$ has a rotation number. For every real number $\omega$, there is an $\bar{f}$-orbit in $M_{\bar{f}}$ whose rotation number is $\omega$.

By the rotation symbol $\widetilde{\rho}(\overline{\mathscr{O}})$ (or $\widetilde{\rho}(\mathscr{O})$ ) of an $\bar{f}$-orbit $\overline{\mathscr{O}}$ in $M_{\bar{f}}$ (or an $f$-orbit $\mathscr{O}$ in $M_{f}$ ), we mean the rotation symbol of the corresponding minimal configuration. As before, the rotation symbol is a real number or has the form $p / q-$ or $p / q+$, where $p / q$ is a rational number.

If $\omega$ is a real number, we let $M_{\bar{f}, \omega}$ denote the union of all $\bar{f}$-orbits in $M_{\bar{f}}$ having rotation symbol (not number) $\omega$. In the case that $\omega$ is a rational number, expressed as $p / q$ in lowest terms with $q>0$, all orbits in $M_{\bar{f}, \omega}$ are periodic of period $q$. In fact, if $\left(\ldots,\left(x_{i}, y_{i}\right), \ldots\right)$ is a lift of such an orbit to $\mathbb{R}^{2}$, then $x_{i+q}=x_{i}+p$ (and consequently $y_{i+q}=y_{i}$ ). This follows immediately from the definition of the rotation symbol, since the Dedekind cut corresponding to the symbol $p / q$ does not contain $p / q$.

If $\omega$ is a rotation symbol of the form $p / q-$ or $p / q+$, we will let $M_{\bar{f}, \omega}$ denote the union of all orbits having rotation symbol either $\omega$ or $p / q$.

Proposition 2.6. If $\bar{f} \in \mathscr{P}_{\beta}^{1}$ and $\omega \in \mathscr{S}$, then $M_{\bar{f}, \omega}$ is a compact subset of the infinite cylinder $(\mathbb{R} / \mathbb{Z}) \times \mathbb{R}$. Let $\pi$ denote the projection of the infinite cylinder on its first factor $\mathbb{R} / \mathbb{Z}$. Then $\pi \mid M_{\bar{f}, \omega}$ is injective. Consequently, $M_{\bar{f}, \omega}=$ graph $u$, for a suitable function $u=u_{\bar{f}, \omega}: \pi\left(M_{\bar{f}, \omega}\right) \rightarrow \mathbb{R}$. This function is Lipschitz with Lipschitz constant $\leq \cot \beta$, i.e., for $\theta, \theta^{\prime} \in \mathbb{R} / \mathbb{Z}$, we have

$$
\left|u\left(\theta^{\prime}\right)-u(\theta)\right| \leq(\cot \beta)\left|\theta^{\prime}-\theta\right| \text {. }
$$

Here, $\left|\theta^{\prime}-\theta\right|$ is defined to be $\min \left|x^{\prime}-x\right|$, where $\theta \equiv x(\bmod 1)$ and $\theta^{\prime} \equiv x^{\prime}(\bmod 1)$.

Proof. It is obvious from the definitions that $M_{\bar{f}, \omega}$ is a closed subset of the infinite cylinder. Various results in Bangert [3] (originally proved in [1] under more restrictive hypotheses) imply that if $x$ and $x^{\prime}$ are minimal configurations corresponding to two orbits in $M_{\bar{f}, \omega}$, then they are comparable. In the case $\omega$ is an irrational number, this is [3, Theorem 4.1]. When $\omega=p / q-, p / q$, or $p / q+$, this is a consequence of [3, Theorems 5.1, and 5.3]: see the discussion at the end of $\S 5$ in [16]. Since any two such configurations are comparable, it follows that $\pi \mid M_{\bar{f}, \omega}$ is injective.

To prove the Lipschitz bound for $u_{\bar{f}, \omega}$, we consider $(x, y)$ and $(\xi, \eta)$ in $M_{f, \omega} \subset \mathbb{R}^{2}$. (Of course, $M_{f, \omega}$ denotes the inverse image of $M_{\bar{f}, \omega}$ under the 
projection $\mathbb{R}^{2} \rightarrow(\mathbb{R} / \mathbb{Z}) \times \mathbb{R}$.) We set $(\bar{x}, \bar{y})=f^{-1}(x, y),(\bar{\xi}, \bar{\eta})=f^{-1}(\xi, \eta)$, $\left(x^{\prime}, y^{\prime}\right)=f(x, y)$, and $\left(\xi^{\prime}, \eta^{\prime}\right)=f(\xi, \eta)$. Suppose, e.g., that $x<\xi$. The comparability result we quoted above implies that $\bar{x}<\bar{\xi}$ and $x^{\prime}<\xi^{\prime}$. We have $y=\partial_{2} h(\bar{x}, x), \eta=\partial_{2} h(\bar{\xi}, \xi)$, where $h$ is the variational principle associated to $f$, by Proposition 2.1. Since $h$ satisfies $\left(\mathrm{H}_{5}\right), \partial_{2} h(\bar{\xi}, x+) \leq \partial_{2} h(\bar{x}, x)$. Since $h$ satisfies $\left(\mathrm{H}_{6 \theta}\right)$, with $\theta=\cot \beta$, we have $\partial_{2} h(\bar{\xi}, \xi) \leq \partial_{2} h(\bar{\xi}, x+)+$ $\theta(\xi-x)$. Combining these inequalities, we get $\eta \leq y+\theta(\xi-x)$. Using $y=-\partial_{1} h\left(x, x^{\prime}\right), \eta=-\partial_{1} h\left(\xi, \xi^{\prime}\right)$, we get $y \leq \eta+\theta(\xi-x)$, by a similar argument.

The fact that minimal configurations corresponding to orbits in $M_{\bar{f}, \omega}$ are comparable has another important consequence. Recall that an ordered triple $\left(\theta, \theta^{\prime}, \theta^{\prime \prime}\right)$ of distinct points in $\mathbb{R} / \mathbb{Z}$ is positively (negatively) oriented if the directed line segment joining $\theta$ to $\theta^{\prime}$ in $\mathbb{R} / \mathbb{Z} \backslash \theta^{\prime \prime}$ is positively (negatively) oriented. This partition of ordered triples of distinct points in $\mathbb{R} / \mathbb{Z}$ into two classes is called the cyclic order of $\mathbb{R} / \mathbb{Z}$. Obviously, it induces a cyclic order on any subset of $\mathbb{R} / \mathbb{Z}$. Since $\pi \mid M_{\bar{f}}, \omega$ is injective, we have a cyclic order on $M_{\bar{f}, \omega}$, too. Likewise, the order on $\mathbb{R}$ induces an order on $M_{f, \omega}$, since the projection of $M_{f, \omega}$ on $\mathbb{R}$ is injective.

Proposition 2.7. $f$ preserves the order on $M_{f, \omega}$ and $\bar{f}$ preserves the cyclic order on $M_{\bar{f}, \omega}$, for any $\omega \in \mathscr{S}$.

Proof. The first statement is a translation of the statement that the configurations corresponding to two orbits in $M_{f, \omega}$ are comparable. The second follows from the first.

Our interest in the subject of minimal orbits was aroused by certain numerical results of Percival [19, 20], who also conjectured [21] (on the basis of numerical evidence) that a homotopically nontrivial invariant circle of a twist mapping consists entirely of minimal orbits. This conjecture very nearly follows from results of Aubry and Le Daeron [1], and we gave a complete proof (for the case of twist maps) in a letter ro R. MacKay [11]. The generalization of this result to the case of finite compositions of twist maps still holds. For the sake of completeness, we next state and prove this generalization:

Proposition 2.8. Let $\bar{f} \in \mathscr{P}^{1}$. Let $\Gamma$ be a subset of the infinite cylinder $(\mathbb{R} / \mathbb{Z}) \times$ $\mathbb{R}$. Suppose that $\Gamma$ is homeomorphic to a circle and is not contractible to a point in the infinite cylinder. Suppose that $\bar{f} \Gamma=\Gamma$. Then $\Gamma \subset M_{\bar{f}}$.

Proof. By a theorem of G. D. Birkhoff (Appendix 1) $\Gamma$ is the graph of a Lipschitz function $u:(\mathbb{R} / \mathbb{Z}) \rightarrow \mathbb{R}$. Let $h$ be a variational principle for $\bar{f}$ and set

$$
H\left(x, x^{\prime}\right)=h\left(x, x^{\prime}\right)-\int_{x}^{x^{\prime}} u(t) d t .
$$

Note that a configuration is minimal with respect to $H$ if and only if it is 
minimal with respect to $h$, because

$$
H\left(x_{i}^{*}, \ldots, x_{j}^{*}\right)-H\left(x_{i}, \ldots, x_{j}\right)=h\left(x_{i}^{*}, \ldots, x_{j}^{*}\right)-h\left(x_{i}, \ldots, x_{j}\right),
$$

if $x_{i}^{*}=x_{i}$ and $x_{j}^{*}=x_{j}$. Moreover, we have

Lemma 1. $H$ is constant on the image of $\Gamma$ in the $\left(x, x^{\prime}\right)$-plane.

By the image of $\Gamma$ in the $\left(x, x^{\prime}\right)$-plane, we mean the following: We assume that a lift $f: \mathbb{R}^{2} \rightarrow \mathbb{R}^{2}$ of $\bar{f}$ is fixed throughout the discussion. We let $\widetilde{\Gamma}$ be the inverse image of $\Gamma$ under the projection of the plane on the infinite cylinder. If $(x, y) \in \mathbb{R}^{2}$ and $f(x, y)=\left(x^{\prime}, y^{\prime}\right)$, we set $\pi_{f}(x, y)=\left(x, x^{\prime}\right)$. By the image of $\widetilde{\Gamma}$ in the $\left(x, x^{\prime}\right)$ plane, we mean $\pi_{f}(\widetilde{\Gamma})$, which we will denote by $\Gamma^{*}$. Note that $\pi_{f}$ is injective on $\widetilde{\Gamma}$ and that $\Gamma^{*}$ is a closed subset of $\mathbb{R}^{2}:$ the first is a consequence of Birkhoff's theorem that $\Gamma$ is a graph.

Proof of Lemma 1. Since $\bar{f} \in \mathscr{P}^{1}$, there exist $\bar{f}_{1}, \ldots, \bar{f}_{k} \in \mathscr{C}^{1}$ such that $\bar{f}=\bar{f}_{k} \circ \cdots \circ \bar{f}_{1}$. We may choose lifts $f_{i}: \mathbb{R}^{2} \rightarrow \mathbb{R}^{2}$ of $\bar{f}_{i}($ for $i=1, \ldots, k)$, such that $f=f_{k} \circ \cdots \circ f_{1}$. Let $\Gamma_{0}=\Gamma, \Gamma_{i}=\bar{f}_{i} \Gamma_{i-1}($ for $i=1, \ldots, k)$. By Birkhoff's theorem, each $\Gamma_{i}$ is the graph of a Lipschitz function $u_{i}: \mathbb{R} / \mathbb{Z} \rightarrow \mathbb{R}$, since $\Gamma_{i}$ is invariant under $\bar{f}_{i} \circ \bar{f}_{i-1} \circ \cdots \circ \bar{f}_{i} \circ \bar{f}_{k} \circ \cdots \circ \bar{f}_{i+1} \in \mathscr{P}^{1}$.

Consider $\left(x, x^{\prime}\right) \in \Gamma^{*}$. Then there exist $y, y^{\prime} \in \mathbb{R}$ such that $(x, y) \in \Gamma$ and $f(x, y)=\left(x^{\prime}, y^{\prime}\right)$. Next, we show that $h$ is differentiable at $\left(x, x^{\prime}\right)$ and

$$
y=-\partial_{1} h\left(x, x^{\prime}\right), \quad y^{\prime}=\partial_{2} h\left(x, x^{\prime}\right) .
$$

To prove this, we recall that although $h$ is not, in general, differentiable, the onesided first partial derivatives $\partial_{1} h\left(x-, x^{\prime}\right)$, etc. always exist and in the example at the end of $[16, \S 5]$, we have given the following expression for them: Let $h_{i}$ be the generating function for $f_{i}$. Among all sequences $x_{0}, \ldots, x_{k}$ that minimize

$$
h_{1}\left(x_{0}, x_{1}\right)+h_{2}\left(x_{1}, x_{2}\right)+\cdots+h_{k}\left(x_{k-1}, x_{k}\right)
$$

subject to the conditions $x_{0}=x$ and $x_{k}=x^{\prime}$, there is a least one, $x_{0}^{\min }, \ldots$, $x_{k}^{\min }$, and a greatest one, $x_{0}^{\max }, \ldots, x_{k}^{\max }$. Let

$$
\begin{array}{ll}
y_{i}^{\mu}=-\partial_{1} h_{i}\left(x_{i}^{\mu}, x_{i+1}^{\mu}\right), & i=0, \ldots, k-1, \\
y_{i}^{\mu}=\partial_{2} h_{i}\left(x_{i}^{\mu}, x_{i+1}^{\mu}\right), & i=1, \ldots, k,
\end{array}
$$

where $\mu=\min$ or $\max$. Then $f_{i}\left(x_{i}^{\mu}, y_{i}^{\mu}\right)=\left(x_{i+1}^{\mu}, y_{i+1}^{\mu}\right)$ and

$$
\begin{array}{ll}
\partial_{1} h\left(x-, x^{\prime}\right)=-y_{0}^{\min }, & \partial_{1} h\left(x+, x^{\prime}\right)=-y_{0}^{\max }, \\
\partial_{2} h\left(x, x^{\prime}-\right)=y_{k}^{\min }, & \partial_{2} h\left(x, x^{\prime}+\right)=y_{k}^{\max } .
\end{array}
$$

Returning to the case $\left(x, x^{\prime}\right) \in \Gamma^{*},(x, y) \in \Gamma$ and $f(x, y)=\left(x^{\prime}, y^{\prime}\right)$, we may use Birkhoff's theorem to prove that $y_{0}^{\min }=y_{0}^{\max }=y$ and $y_{k}^{\min }=$ $y_{k}^{\max }=y^{\prime}$. For, suppose to the contrary that $y_{0}^{\mu} \neq y(\mu=\min$ or $\max )$, e.g., 
$y_{0}^{\mu}<y$. Let $\left(x_{0}, y_{0}\right)=(x, y),\left(x_{i}, y_{i}\right)=f_{i}\left(x_{i-1}, y_{i-1}\right), i=1, \ldots, k$. The twist condition on $f_{1}$ implies $x_{1}^{\mu}<x_{1}$, since $x_{0}^{\mu}=x_{0}$ and $y_{0}^{\mu}<y_{0}$. Clearly $y_{1}^{\mu}<u_{1}\left(x_{1}^{\mu}\right)$, i.e., $\left(x_{1}^{\mu}, y_{1}^{\mu}\right)$ is below $\Gamma_{1}=$ graph $u_{1}$. The twist condition on $f_{2}$, together with the fact that $\Gamma_{1}$ and $\Gamma_{2}$ are graphs of functions, then implies that $x_{2}^{\mu}<x_{2}$. Continuing in this way, we obtain, after $k$ steps, $x_{k}^{\mu}<x_{k}$. But, by definition, we have $x_{k}^{\mu}=x^{\prime}=x_{k}$. This contradiction shows that $y_{0}^{\mu}<y$ is impossible. Similarly, $y_{0}^{\mu}>y$ implies $x_{k}^{\mu}>x_{k}$, also a contradiction.

Thus, we have shown that $y_{0}^{\min }=y_{0}^{\max }=y$ and $y_{k}^{\min }=y_{k}^{\max }=y^{\prime}$. In view of our formula for the one-sided first partial derivatives, this shows that $h$ is differentiable at $\left(x, x^{\prime}\right)$ and $u(x)=y=-\partial_{1} h\left(x, x^{\prime}\right), u\left(x^{\prime}\right)=y^{\prime}=$ $\partial_{2} h\left(x, x^{\prime}\right)$.

For each $x \in \mathbb{R}$, there is a unique $x^{\prime}=x^{\prime}(x)$ such that $\left(x, x^{\prime}\right) \in \Gamma^{*}$. By Birkhoff's theorem and the assumption that $f$ is $C^{1}, x^{\prime}$ is a Lipschitz function of $x$, and consequently is differentiable almost everywhere. (See, e.g., $[22, \S 11.7]$.) Then

But,

$$
\frac{d}{d x} H\left(x, x^{\prime}(x)\right)=\partial_{1} H\left(x, x^{\prime}\right)+\partial_{2} H\left(x, x^{\prime}\right) \frac{d x^{\prime}}{d x} .
$$

$$
\begin{aligned}
& \partial_{1} H\left(x, x^{\prime}\right)=\partial_{1} h\left(x, x^{\prime}\right)+u(x)=0, \\
& \partial_{2} H\left(x, x^{\prime}\right)=\partial_{2} h\left(x, x^{\prime}\right)-u\left(x^{\prime}\right)=0 .
\end{aligned}
$$

Thus $d H\left(x, x^{\prime}(x)\right) / d x$ vanishes almost everywhere. Since $H\left(x, x^{\prime}(x)\right)$ is a Lipschitz function of $x$, this implies that $H$ is constant on $\Gamma^{*}$. (See, e.g., [22, $\S 11.7]$.)

Let $C$ be the constant value of $H$ on $\Gamma^{*}$, given by Lemma 1 .

Lemma 2. $C$ is the minimum value of $H$ and this value is taken only on $\Gamma^{*}$. Proof. Obviously, $H\left(\xi, x^{\prime}\right)+H\left(x, \xi^{\prime}\right)-H\left(x, x^{\prime}\right)-H\left(\xi, \xi^{\prime}\right)=h\left(\xi, x^{\prime}\right)+$ $h\left(x, \xi^{\prime}\right)-h\left(x, x^{\prime}\right)-h\left(\xi, \xi^{\prime}\right)$, if $x<\xi$ and $x^{\prime}<\xi^{\prime}$. Consequently, $H$ satisfies $\left(\mathrm{H}_{5}\right)$. It follows that

$$
\liminf _{\xi \downarrow x} \frac{H\left(\xi, x^{\prime}\right)+H\left(x, \xi^{\prime}\right)-H\left(x, x^{\prime}\right)-H\left(\xi, \xi^{\prime}\right)}{\xi-x} \geq \int_{x^{\prime}}^{\xi^{\prime}} \rho(x, t) d t,
$$

if $x^{\prime}<\xi^{\prime}$. In the proof of Lemma 1, we showed that $\partial_{1} H\left(x, x^{\prime}\right)=0$, when $\left(x, x^{\prime}\right) \in \Gamma^{*}$. Therefore,

$$
\limsup _{\xi \downarrow x} \frac{H\left(\xi, \xi^{\prime}\right)-H\left(x, \xi^{\prime}\right)}{\xi-x} \leq-\int_{x^{\prime}}^{\xi^{\prime}} \rho(x, t) d t,
$$

when $\left(x, x^{\prime}\right) \in \Gamma^{*}$ and $x^{\prime}<\xi^{\prime}$. We may treat limsup when $\xi$ tends upward to $x$ in the same way, and obtain the same upper bound. Combining the two cases, we get

$$
\limsup _{\xi \rightarrow x} \frac{H\left(\xi, \xi^{\prime}\right)-H\left(x, \xi^{\prime}\right)}{\xi-x} \leq-\int_{x^{\prime}}^{\xi^{\prime}} \rho(x, t) d t,
$$


when $\left(x, x^{\prime}\right) \in \Gamma^{*}$ and $x^{\prime}<\xi^{\prime}$. In other words, the upper Dini derivate of $H\left(x, \xi^{\prime}\right)$ with respect to the first variable is negative when $\left(x, \xi^{\prime}\right)$ is above $\Gamma^{*}$. A similar argument shows that the lower Dini derivate of $H\left(x, \xi^{\prime}\right)$ with respect to the first variable is positive when $\left(x, \xi^{\prime}\right)$ is below $\Gamma^{*}$.

Let $\left(x, \xi^{\prime}\right) \in \mathbb{R}^{2}$ and let $\xi$ be the unique real number such that $\left(\xi, \xi^{\prime}\right) \in \Gamma^{*}$. If $\left(x, \xi^{\prime}\right)$ is above (resp. below) $\Gamma^{*}$, then $x<\xi$ (resp. $x>\xi$ ). Lemma 2 follows from Lemma 1 and the assertions we just made about the Dini derivates of $H$ with respect to the first variable, since these show that $H\left(x, \xi^{\prime}\right)>H\left(\xi, \xi^{\prime}\right)$, if $x \neq \xi$.

Proof of Proposition 2.8 (Conclusion). Let $\left(\ldots,\left(x_{i}, y_{i}\right), \ldots\right)$ be an $f$-orbit in $\widetilde{\Gamma}$. The corresponding configuration satisfies $\left(x_{i}, x_{i+1}\right) \in \Gamma^{*}$, for every $i$. It follows immediately from Lemma 2 that this configuration is minimal for $H$ and hence also minimal for $h$.

J. Moser pointed out to me that Proposition 2.8 is closely related to a famous theorem of Weierstrass that asserts that elements of a field of extremals of a positive definite Lagrangian system minimize the action, and that the proof we have given above is closely related to Weierstrass's proof. See Carathéodory [5] for the statement of this result, and its proof.

\section{THE INTRINSIC NATURE OF $M_{\bar{f}}$}

It is not at all obvious from the definition of $M_{\bar{f}}$, which we gave in the previous section, but $M_{\bar{f}}$ is intrinsic, in the sense stated in the following result:

Proposition 3.1. Let $\bar{f}, \bar{g} \in \mathscr{P}^{1}$ and suppose that there exists a $C^{1}$ areapreserving diffeomorphism $\bar{\phi}$ of the infinite cylinder $(\mathbb{R} / \mathbb{Z}) \times \mathbb{R}$ such that $\bar{g}=$ $\overline{\phi f \phi}^{-1}$. Then $\bar{\phi}\left(M_{\bar{g}}\right)=M_{\bar{f}}$.

The rest of this section is a proof of Proposition 3.1.

We begin by showing that $\bar{\phi}$ is orientation-preserving. Let $C>0$. There are orbits of $\bar{f}$ of rotation number $>C$ (resp. $<-C$ ) in every neighborhood of the top (resp. bottom) end of the infinite cylinder. This follows from the theory of the previous section: $M_{\bar{f}}$ contains such orbits. On the other hand, if $N$ is a sufficiently small neighborhood of the top (resp. bottom) end, every orbit that is contained entirely in $N$ and has a rotation number, has rotation number $>C$ (resp. $<-C$ ). This is because $\bar{f}$ twists the ends infinitely.

Recall that the definition of the rotation number depends on a choice of lift; we choose the lifts $f$ of $\bar{f}$ and $g$ of $\bar{g}$ such that $g=\phi f \phi^{-1}$, where $\phi$ is a lift of $\bar{\phi}$. With this convention, if $\bar{\phi}$ induces the identity on $\pi_{1}((\mathbb{R} / \mathbb{Z}) \times \mathbb{R})$, then $\bar{\phi}$ carries an orbit of $\bar{g}$ to an orbit of $\bar{f}$ with the same rotation number; if $\bar{\phi}$ induces the nontrivial automorphism of $\pi_{1}((\mathbb{R} / \mathbb{Z}) \times \mathbb{R})$, then $\bar{\phi}$ carries an orbit of $\bar{f}$ to an orbit of $\bar{g}$ whose rotation number is the negative of the rotation number of the original orbit. 
Thus, either $\bar{\phi}$ induces the identity on $\pi_{1}$ and fixes the ends, or it induces the negative of the identity and reverses the ends. In either case $\bar{\phi}$ is orientationpreserving.

Throughout the rest of this section, we let $\bar{f}$ be an element of $\mathscr{P}^{1}$. We will show that $M_{\bar{f}}$ can be defined in terms of $\bar{f}$ and the area form $\Omega=d y \wedge d \theta$ on the infinite cylinder, alone. This will be enough, since $\bar{\phi}$ is orientation- and area-preserving and therefore preserves the area form.

For an $\bar{f}$-invariant probability measure $\mu$, we defined the average action (or average Poincaré-Cartan invariant) $A(F, \mu)=A_{H, \eta}(f, \mu)$ in [18]. This depends on a choice of 1-form $\eta$ such that $d \eta=\Omega$ and a periodic Hamiltonian $H$ (of period one) such that $\bar{f}$ is the time-one map associated to $H$. But this has the usual invariance properties of the Poincare-Cartan invariant. In the present situation, these invariance properties amount to the following: if $H^{\prime}$ is a second periodic Hamiltonian (of period one) whose time-one map is $\bar{f}$ and $\eta^{\prime}$ is a second 1 -form such that $d \eta^{\prime}=\Omega$, then there exist $B, C \in \mathbb{R}$ such that

$$
A_{H^{\prime}, \eta^{\prime}}(\bar{f}, \mu)=A_{H, \eta}(\bar{f}, \mu)+B \rho(f, \mu)+C,
$$

for every $\bar{f}$-invariant probability measure $\mu$. Here, $\rho(f, \mu)$ denotes the average rotation number of $\mu$, also defined in [18]. The above invariance property of the average action was proved in [18].

In [18], we defined the notion of a minimal measure: an $\bar{f}$-invariant probability measure $\mu$ is minimal if and only if there exists a real number $\lambda$ such that $\mu$ minimizes $A(\bar{f}, \mu)-\lambda \rho(f, \mu)$, over all $\bar{f}$-invariant probability measures $\mu$. This notion depends only on the area form $\Omega$ and $\bar{f}$, by the above invariance property of the average action.

The main result of [18] was that an ergodic $\bar{f}$-invariant probability measure $\mu$ is minimal if and only if its support lies in $M_{\bar{f}}$. Let $M_{\bar{f}}^{\circ}$ denote the closure of the union of all supports of all $\bar{f}$-minimal measures. Since the notion of minimal measure depends only on $\Omega$ and $\bar{f}$, so does $M_{\bar{f}}^{\circ}$. In other words, $M_{\bar{f}}^{\circ}$ is intrinsic in the sense of Proposition 3.1, i.e., $\bar{\phi}\left(M_{\bar{g}}^{\circ}\right)=M_{\bar{f}}^{\circ}$.

Here is an outline of our proof that $M_{\bar{f}}$ is intrinsic. We denote by $M_{f}^{\circ}$ the inverse image of $M_{\bar{f}}^{\circ}$ in $\mathbb{R}^{2}$ (by analogy with $M_{f}$, the inverse image of $M_{\bar{f}}$ in $\left.\mathbb{R}^{2}\right)$. We will show below that if $\mathscr{O}=\left(\ldots,\left(x_{i}, y_{i}\right), \ldots\right)$ is an orbit in $M_{f}$, then there is an orbit $\mathscr{O}^{\prime}=\left(\ldots,\left(x_{i}^{\prime}, y_{i}^{\prime}\right), \cdots\right)$ in $M_{f}^{\circ}$ that is $L^{1}$-asymptotic to $\mathscr{O}$, in the sense that $\sum_{i=-\infty}^{\infty}\left|x_{i}^{\prime}-x_{i}\right|+\left|y_{i}^{\prime}-y_{i}\right|<+\infty$. When $\mathscr{O}$ and $\mathscr{O}^{\prime}$ are $L^{1}$-asymptotic orbits of $f$ whose projections on the infinite cylinder are relatively compact, we will define a difference action (or difference PoincaréCartan invariant $) \Delta A\left(\mathscr{O}, \mathscr{O}^{\prime}\right)$, an intrinsic notion. We will show that orbits $\mathscr{O}$ of $M_{f}$ are characterized by the following property: there is an orbit $\mathscr{O}^{\prime}$ in $M_{f}^{\circ}$ that is $L^{1}$-asymptotic to $\mathscr{O}$ such that $\Delta A\left(\mathscr{O}, \mathscr{O}^{\prime}\right)=0$. Since $M_{f}^{\circ}$ is intrinsic, this will show that $M_{f}^{\circ}$ is intrinsic. 
Let $\mathscr{O}=\left(\ldots,\left(x_{i}, y_{i}\right), \ldots\right)$ be an orbit in $M_{f}$. Let $\omega$ be the rotation symbol of $\mathscr{O}$ and set $M_{f, \omega}^{\circ}=M_{f}^{\circ} \cap M_{f, \omega}$. Let $x=\left(\ldots, x_{i}, \ldots\right)$ denote the configuration corresponding to $\mathscr{O}$, and let $X_{f, \omega}^{\circ}$ denote the set of configurations corresponding to orbits in $M_{f, \omega}^{\circ}$. We have seen (Proposition 2.7) that Aubry's theory implies that any two elements of $X_{f, \omega}^{\circ}$ are comparable. Since $M_{f, \omega}^{\circ}$ is also closed in $\mathbb{R}^{2}$, it follows that there is a greatest element $x_{-}=\left(\ldots, x_{i-}, \ldots\right)$ of $X_{f, \omega}^{\circ}$ that is $\leq x$ and a least element $x_{+}=\left(\ldots, x_{i+}, \ldots\right)$ of $X_{f, \omega}^{\circ}$ that is $\geq x$. Let $\mathscr{O}_{-}$and $\mathscr{O}_{+}$denote the corresponding orbits (in $M_{f, \omega}^{\circ}$ ).

Then $\sum_{i=-\infty}^{\infty} x_{i+}-x_{i-} \leq 1$. This is because the projections of the intervals $\left[x_{i-}, x_{i+}\right]$ on $\mathbb{R} / \mathbb{Z}$ are mutually disjoint if the rotation symbol $\omega$ is not a rational number $p / q$ and $x_{-}=x=x_{+}$if $\omega$ is a rational number $p / q$. In fact, if $\omega=p / q$, then $x_{i+q}=x_{i}+p$, so the projection of $\mathscr{O}$ on $(\mathbb{R} / \mathbb{Z}) \times \mathbb{R}$ is periodic of period $q$ and carries an ergodic probability measure, so $\mathscr{O}$ is in $M_{f, p / q}^{\circ}$ in this case, and consequently $x_{-}=x=x_{+}$, as asserted. When $\omega$ is an irrational number, the fact that the projections on $\mathbb{R} / \mathbb{Z}$ of the intervals $\left[x_{i-}, x_{i+}\right]$ are mutually disjoint is an obvious consequence of the cyclic orderpreserving property of $\bar{f} \mid M_{\bar{f}, \omega}$ (Proposition 2.7). (Recall that $M_{\bar{f}, \omega}$ supports exactly one invariant probability measure $\mu_{\bar{f}, \omega}$ and $\operatorname{supp} \mu_{\bar{f}, \omega} \subset M_{\bar{f}}^{\circ},{ }^{\circ}$.)

In the remaining cases, i.e., when $\omega=p / q-$ or $p / q+$, this fact is a little more subtle. For simplicity, we consider only the case $\omega=p / q+$, the other case being similar. An argument of Aubry (see Bangert [3, Theorem 5.8]) shows that $M_{f, p / q+}^{\circ}$ meets every complementary interval of $M_{f, p / q}^{\circ}=M_{f, p / q}$; this is because $M_{\bar{f}, p / q+}^{\circ} \supset \lim \sup _{i \rightarrow \infty} \operatorname{supp} \mu_{\bar{f}, \lambda(i)}$, for any sequence of irrational numbers $\lambda_{i}$ tending downwards to $p / q$. Since $M_{f, p / q+}^{\circ}$ meets every complementary interval of $M_{f, p / q}^{\circ}$, it follows that $x_{-}$and $x_{+}$have rotation symbol $p / q+($ not $p / q)$. It is then an easy consequence of the cyclic order-preserving property of $\bar{f} \mid M_{\bar{f}, p / q+}$ (Proposition 2.7), together with the defining property of the rotation symbol $p / q+$, that the projections on $\mathbb{R} / \mathbb{Z}$ of the intervals $\left[x_{i-}, x_{i+}\right]$ are mutually disjoint.

Let $\mathscr{O}^{\prime}=\mathscr{O}_{-}, x^{\prime}=x_{-}$, etc. Since $x_{-} \leq x \leq x_{+}$, we have $\sum_{i=-\infty}^{\infty}\left|x_{i}-x_{i}^{\prime}\right| \leq$ 1 , by what we have just shown. Since $\mathscr{O}$ and $\mathscr{O}^{\prime}$ are minimal orbits, we have $y_{i}=-\partial_{1} h\left(x_{i}, x_{i+1}\right)=\partial_{2} h\left(x_{i-1}, x_{i}\right)$ and $y_{i}^{\prime}=-\partial_{1} h\left(x_{i}^{\prime}, x_{i+1}^{\prime}\right)=\partial_{2} h\left(x_{i-1}^{\prime}, x_{i}^{\prime}\right)$. From $\left(\mathrm{H}_{5}\right)$ and $\left(\mathrm{H}_{6 \theta}\right)$ and $x^{\prime}<x$, we may obtain $\left|y_{i}-y_{i}^{\prime}\right| \leq \theta\left|x_{i}-x_{i}^{\prime}\right|$. Thus, $\mathscr{O}$ and $\mathscr{O}^{\prime}$ are $L^{1}$-asymptotic. This completes the first step: we have shown that any orbit $\mathscr{O}$ in $M_{f}$ is $L^{1}$-asymptotic to an orbit $\mathscr{O}^{\prime}$ in $M_{f}^{\circ}$.

If $\mathscr{O}$ and $\mathscr{O}^{\prime}$ are $L^{1}$-asymptotic orbits of $f$ whose projections on the infinite cylinder are relatively compact, then we may define a difference action (or 
difference Poincaré-Cartan invariant)

$$
A\left(\mathscr{O}^{\prime}, \mathscr{O}\right)=\lim _{\substack{i \rightarrow-\infty \\ j \rightarrow \infty}} L_{\eta}\left(\mathscr{O}^{\prime}\right)(i, j)-L_{\eta}(\mathscr{O})(i, j) .
$$

The quantity on the right is defined as follows: It is known that $\bar{f}$ is the timeone map of the flow generated by a periodic Hamiltonian $H$ of period one on the infinite cylinder (see, e.g., [18, §3]). If $\Gamma$ is the trajectory of $H$ (in the infinite cylinder) extending $\mathscr{O}$, we set

$$
L_{\eta}(\mathscr{O})(i, j)=\int_{\Gamma \mid[i, j]} \eta-H d t
$$

Here, $\eta$ is any 1 -form on the infinite cylinder such that $d \eta=d y \wedge d \theta$. This is the same as the notation in $[18, \S 2]$, and we refer the reader to that article for a fuller explanation. In particular, we may take $\eta=y d \theta$. Thus, if we let $\Gamma^{\prime}$ be the trajectory associated to $\mathscr{O}^{\prime}$ and set $\Gamma(t)=(\theta(t), y(t)), \Gamma^{\prime}(t)=$ $\left(\theta^{\prime}(t), y^{\prime}(t)\right)$, we have

$$
\Delta A\left(\mathscr{O}^{\prime}, \mathscr{O}\right)=\int_{-\infty}^{\infty} d t\left[y^{\prime} \frac{d \theta^{\prime}}{d t}-y \frac{d \theta}{d t}-H(\theta, y, t)-H\left(\theta^{\prime}, y^{\prime}, t\right)\right] .
$$

Since $\mathscr{O}$ and $\mathscr{O}^{\prime}$ are $L^{1}$-asymptotic, the quantity under the integral sign is in $L^{1}(\mathbb{R})$, so the integral on the right exists.

In $[18, \S 2]$, we analyzed how $L_{\eta}(\mathscr{O})(i, j)$ depends on $H$ and $\eta$. Changing $H$ changes this only by a constant of integration. Replacing $\eta$ by $\eta^{\prime}$ changes it by $\int_{\Gamma \mid[i, j]} \eta^{\prime}-\eta$. Since $\eta^{\prime}-\eta$ is closed, it follows that $\Delta A\left(\mathscr{O}^{\prime}, \mathscr{O}\right)$ does not depend on $\eta$ (as long as $d \eta$ is the area form) or on $H$. In other words, it is intrinsic.

Choose $\bar{f}_{1}, \ldots, \bar{f}_{k} \in \mathcal{J}^{1}$ such that $\bar{f}=\bar{f}_{k} \circ \ldots \circ \bar{f}_{1}$. We let $f_{i}$ be a lift of $\bar{f}_{i}$ to the universal cover and $f=f_{k} \circ \cdots \circ f_{1}$. We let $h_{i}$ be a generating function associated to $f_{i}$. We may suppose that these various choices are made so that $h=h_{1} * \cdots * h_{k}$. We set

$$
\left(x^{(k i+j)}, y^{(k i+j)}\right)=f_{j} \circ \cdots \circ f_{1}\left(x_{i}, y_{i}\right)
$$

and we use the similar notation (with primes) for $\mathscr{O}^{\prime}$ in place of $\mathscr{O}$. We have

$$
\Delta A\left(\mathscr{O}^{\prime}, \mathscr{O}\right)=\sum_{i=-\infty}^{\infty} h_{i}\left(x^{\prime(i)}, x^{\prime(i+1)}\right)-h_{i}\left(x^{(i)}, x^{(i+1)}\right),
$$

where we extend the definition of $h_{i}$ to all $i \in \mathbb{Z}$, by setting $h_{i+k}=h_{i}$. This formula holds by the argument of $[18, \S 2]$.

From this formula, it follows that if $\mathscr{O}$ is a minimal orbit and $\mathscr{O}^{\prime}$ is $L^{1}$ asymptotic to $\mathscr{O}$, then $\Delta A\left(\mathscr{O}^{\prime}, \mathscr{O}\right) \geq 0$ with equality if and only if $\mathscr{O}^{\prime}$ is also minimal.

It follows that the orbits in $M_{f}$ have the following intrinsic characterization: they are the orbits $\mathscr{O}$ for which there exists an orbit $\mathscr{O}^{\prime}$ in $M_{f}^{\circ}$ that is $L^{1}$ asymptotic to $\mathscr{O}$ and that satisfies $\Delta A\left(\mathscr{O}, \mathscr{O}^{\prime}\right)=0$.

This proves Proposition 3.1. 


\section{THE MAIN RESULTS}

In this section, we state the main results of this paper. Most of the rest of this paper will consist of their proofs.

We let $\bar{f}$ be a member of $\mathscr{P}^{1}$, which will be fixed throughout the rest of this paper. By a Birkhoff region of instability $R$ for $\bar{f}$, we mean a compact $\bar{f}$ invariant subset of the infinite cylinder, with the following properties: First, the frontier of $R$ consists of two components $\Gamma_{-}$and $\Gamma_{+}$, each homeomorphic to a circle and each noncontractible to a point in the infinite cylinder. Second, if $\Gamma$ is any $\bar{f}$-invariant subset of $R$, homeomorphic to a circle, and noncontractible to a point in the infinite cylinder, then $\Gamma$ is $\Gamma_{-}$or $\Gamma_{+}$.

Note that since $\bar{f}$ fixes the ends of the infinite cylinder, each of $\Gamma_{-}$and $\Gamma_{+}$ is $\bar{f}$-invariant. It follows from Proposition 2.8 that $\Gamma_{ \pm} \subset M_{\bar{f}}$. Throughout the rest of this paper, we let $R$ denote a fixed Birkhoff region of instability of $\bar{f}$. We let $\Gamma_{-}$denote the lower component of the boundary of $R$ and $\Gamma_{+}$the upper component.

Here are the main results:

Theorem 4.1. If $\rho\left(\Gamma_{-}\right) \leq \omega_{-}, \omega_{+} \leq \rho\left(\Gamma_{+}\right)$, then there is an $\bar{f}$-orbit $\mathscr{O}$ in $R$ such that $\mathscr{O}$ is $\alpha$-asymptotic to $M_{\bar{f}, \omega_{-}}$and $\omega$-asymptotic to $M_{\bar{f}, \omega_{+}}$, provided that if $\omega_{-}=\rho\left(\Gamma_{-}\right)$(resp. $\left.\omega_{+}=\rho\left(\Gamma_{+}\right)\right)$, then $\omega_{-}$(resp. $\left.\omega_{+}\right)$is irrational.

This means the following: Let $\mathscr{O}=\left(\ldots,\left(\theta_{i}, y_{i}\right), \ldots\right)$. The statement that $\mathscr{O}$ is $\alpha$-asymptotic to $M_{\bar{f}, \omega_{-}}$means that $\operatorname{dist}\left(\left(\theta_{i}, y_{i}\right), M_{\bar{f}, \omega_{-}}\right) \rightarrow 0$ as $i \rightarrow-\infty$. The statement that $\mathscr{O}$ is $\omega$-asymptotic to $M_{\bar{f}, \omega_{+}}$means that $\operatorname{dist}\left(\left(\theta_{i}, y_{i}\right), M_{\bar{f}, \omega_{+}}\right) \rightarrow 0$ as $i \rightarrow+\infty$.

Theorem 4.2. Consider for each $i \in \mathbb{Z}$ a real number $\rho\left(\Gamma_{-}\right) \leq \omega_{i} \leq \rho\left(\Gamma_{+}\right)$ and a positive number $\varepsilon_{i}$. There exists an $\bar{f}$-orbit $\mathscr{O}=\left(\ldots,\left(\theta_{j}, y_{j}\right), \ldots\right)$ in $R$ and an increasing bi-infinite sequence $\ldots, j(i), \ldots$ of integers such that $\operatorname{dist}\left(\left(\theta_{j(i)}, y_{j(i)}\right), M_{\bar{f}, \omega(i)}\right)<\varepsilon_{i}$.

In other words, $\mathscr{O}$ approaches to within $\varepsilon_{i}$ of $M_{\bar{f}, \omega(i)}$ at the $j_{i}$ th iteration.

\section{5. $\mathcal{J}$-Minimal CONFIGURATIONS}

By a constraint $\mathscr{J}$, we will mean a bi-infinite sequence $\left(\ldots, J_{i}, \ldots\right)$, where each $J_{i}$ is a closed, connected, nonempty subset of $\mathbb{R}$. By a $\mathscr{J}$-configuration, we will mean a bi-infinite sequence $\left(\ldots, x_{i}, \ldots\right)$ with $x_{i} \in J_{i}$. By a segment of a $\mathscr{J}$-configuration, we will mean a finite sequence $\left(x_{j}, \ldots, x_{k}\right)$ such that $x_{i} \in J_{i}$, for each $j \leq i \leq k$.

Throughout the rest of this paper, we consider a fixed lift $f$ of $\bar{f}$ to the universal cover. We let $h$ be a variational principle associated to $f$.

A segment of a $\mathscr{J}$-configuration $\left(x_{j}, \ldots, x_{k}\right)$ will be said to be $\mathscr{J}$-minimal (with respect to $h$ ) if

$$
h\left(x_{j}, \ldots, x_{k}\right) \leq h\left(x_{j}^{*}, \ldots, x_{k}^{*}\right)
$$


for every segment of a $\mathscr{J}$-configuration $\left(x_{j}^{*}, \ldots, x_{k}^{*}\right)$ such that $x_{j}^{*}=x_{j}$ and $x_{k}^{*}=x_{k}$. We will say that a $\mathscr{J}$-configuration $\left(\ldots, x_{i}, \ldots\right)$ is $\mathscr{J}$-minimal if for every $j<k$, the corresponding segment $\left(x_{j}, \ldots, x_{k}\right)$ of it is $\mathscr{J}$-minimal.

The following two propositions, although almost obvious, are fundamental to the structure of our proofs of the main results.

Proposition 5.1. Let $\mathscr{J}=\left(\ldots, J_{i}, \ldots\right)$ be a constraint such that there exist arbitrarily small and arbitrarily large $i$ for which $J_{i}$ is bounded. Then there exists a $\mathscr{J}$-minimal configuration.

Proof. By $\left(\mathrm{H}_{2}\right)$, the function $h\left(x_{-N}, x_{-N+1}, \ldots, x_{N}\right)$ is proper, continuous and bounded below on $J_{-N} \times J_{-N+1} \times \cdots \times J_{N}$. It follows that there is a sequence $\left(x_{-N}^{(N)}, x_{-N+1}^{(N)}, \ldots, x_{N}^{(N)}\right)$ that minimizes this function over $J_{-N} \times J_{-N+1} \times \cdots \times$ $J_{N}$. Using $\left(\mathrm{H}_{2}\right)$, one may find, for each integer $j$, a compact set $K_{j}$ such that $x_{j}^{(N)} \in K_{j}$ for all $N$ : if $J_{j}$ is bounded one takes $K_{j}=J_{j}$. Otherwise one uses the fact that there exist $j^{\prime}<j<j^{\prime \prime}$ for which $J_{j^{\prime}}$ and $J_{j^{\prime \prime}}$ are bounded, and property $\left(\mathrm{H}_{2}\right)$ of $h$. It follows, by the Cantor diagonal process, that we may choose a sequence $N_{1}<N_{2}<\cdots$ such that $x_{j}^{N(i)} \rightarrow x_{j} \in K_{j}$, as $i \rightarrow+\infty$. It is easily verified that the resulting bi-infinite sequence $\left(\ldots, x_{j}, \ldots\right)$ is $\mathscr{J}$ minimal.

We will say that a $\mathscr{J}$-configuration $x=\left(\ldots, x_{i}, \ldots\right)$ is $\mathscr{J}$-free if $x_{i} \in$ int $J_{i}$, for each $i$.

Proposition 5.2. Let $x=\left(\ldots, x_{i}, \ldots\right)$ be a $\mathscr{J}$-minimal configuration. If $x$ is $\mathcal{J}$-free, then $-\partial_{1} h\left(x_{i}, x_{i+1}\right)=\partial_{2} h\left(x_{i-1}, x_{i}\right)$. (In particular, these partial derivatives exist.) Moreover $f\left(x_{i}, y_{i}\right)=\left(x_{i+1}, y_{i+1}\right)$, where $y_{i}=-\partial_{1} h\left(x_{i}, x_{i+1}\right)$, i.e., $\left(\ldots,\left(x_{i}, y_{i}\right), \ldots\right)$ is an $f$-orbit.

Proof. This is identical to the discussion of minimal orbits in $\S 2$.

According to Proposition 5.1, $\mathscr{J}$-minimal configurations exist; according to Proposition 5.2, they give rise to orbits if they are $\mathscr{J}$-free. The orbits whose existence is asserted in Theorems 4.1 and 4.2 will be constructed as the orbits associated to $\mathscr{J}$-minimal and $\mathscr{J}$-free configurations. The method of proof consists of showing that if $\mathscr{J}=\left(\ldots, J_{i}, \ldots\right)$ is a properly chosen constraint, then any $\mathscr{J}$-minimal configuration is $\mathscr{J}$-free, and the corresponding orbit has the properties required by Theorem 4.1 or Theorem 4.2. The specifications on $\mathcal{J}$ that produce this result are complicated and will be explained in later sections.

\section{PARTially $\mathscr{J}$-Free CONFigurations}

In this section, we give conditions on a constraint $\mathscr{J}$ that guarantee a $\mathscr{J}$. minimal configuration is at least partially $\mathscr{J}$-free. The principal result of this section is Proposition 6.1. We also give a generalization of Proposition 6.1, Proposition 6.2, which will be needed in the proof of the main results of this paper. 
To state our condition, we need to recall two basic notions related to minimal configurations: Peierls's barrier and the minimizer of Percival's Lagrangian.

Let $\omega$ be a rotation symbol and let $a \in \mathbb{R}$. Let $\bar{\omega}$ be the underlying number of $\omega$ : if $\omega$ is a real number, then $\bar{\omega}=\omega$; if $\omega=p / q \pm$, then $\bar{\omega}=p / q$.

There exist a largest minimal configuration $\xi_{-}=\left(\ldots, \xi_{i-}, \ldots\right)$ of rotation symbol $\omega$ or $\bar{\omega}$ such that $\xi_{0_{-}} \leq a$ and a smallest minimal configuration $\xi_{+}=\left(\ldots, \xi_{i+}, \ldots\right)$ of rotation symbol $\omega$ or $\bar{\omega}$ such that $a \leq \xi_{0+}$. (Here smallest and largest refer to the order on minimal configurations of rotation symbol $\omega$ or $\bar{\omega}$ introduced in $\S 2: \xi<\xi^{\prime}$ if and only if $\xi_{i}<\xi_{i}^{\prime}$ for all integers i.) We have

$$
\sum_{i \in I} \xi_{i+}-\xi_{i-} \leq 1,
$$

where the index set $I$ is the set $\mathbb{Z}$ of integers if $\omega$ is not a rational number and is $\{0, \ldots, q-1\}$ if $\omega=p / q$. This is because either $\xi_{i-}=a=\xi_{i+}$ or the projections on $\mathbb{R} / \mathbb{Z}$ of the intervals $\left(\xi_{i-}, \xi_{i+}\right)$ are mutually disjoint. We set

$$
P_{\omega}^{h}(a)=P_{\omega}(a)=\min \sum_{i \in I} h\left(\xi_{i}, \xi_{i+1}\right)-h\left(\xi_{i-}, \xi_{i+1-}\right) .
$$

Here, the minimum is taken over all $\xi=\left(\ldots, \xi_{i}, \ldots\right)$ such that $\xi_{i-} \leq \xi_{i} \leq \xi_{i+}$ and $\xi_{0}=a$. The condition $\xi_{i-} \leq \xi_{i} \leq \xi_{i+}$ guarantees that the above sum is absolutely convergent.

In [2], $P_{\omega}(a)$ was called Peierls's barrier because of the relation of $P_{\omega}(a)$ with known notions in solid state physics.

Note that $P_{\omega}(a) \geq 0$ for all $a \in \mathbb{R}$ and $P_{\omega}(a)=0$ if and only if $a \in \pi\left(M_{\omega}\right)$, where $\pi$ denotes the projection of $\mathbb{R}^{2}$ on $\mathbb{R}$.

The second notion that we need may be most easily defined in terms of Percival's Lagrangian

$$
P_{\omega}(\phi)=\int_{0}^{1} h(\phi(t), \phi(t+\omega)) d t
$$

This quantity is defined for any bounded and measurable function $\phi$ such that $\phi(t+1)=\phi(t)+1$ and any number $\omega$. We let $\phi_{\omega}$ denote any such function that minimizes $P_{\omega}$. Existence of such a function was proved, for the case of twist mappings, in $[12,15]$.

However, another definition of $\phi_{\omega}$, due to Aubry [1], will be more convenient for this paper. It follows easily from the order property of $M_{f, \omega}$ (Proposition 2.7) that for every real number $\omega$, there exists a function $\phi_{\omega}: \mathbb{R} \rightarrow \mathbb{R}$ such that

(1) $s \leq t$ implies $\phi_{\omega}(s) \leq \phi_{\omega}(t)$;

(2) $\phi_{\omega}(t+1)=\phi_{\omega}(t)+1$

(3) if $t \in \mathbb{R}$, then

$$
x_{-}^{\omega, t}=\left(\ldots, \phi_{\omega}(t+\omega i-), \ldots\right), \quad x_{+}^{\omega, t}=\left(\ldots, \phi_{\omega}(t+\omega i+), \ldots\right)
$$

are minimal configurations of rotation symbol $\omega$. Here

$$
\phi(s-)=\lim _{u \uparrow s} \phi(u), \quad \phi(s+)=\lim _{u \downarrow s} \phi(u) .
$$


It may be shown that a function $\phi_{\omega}$ which satisfies (1)-(3) minimizes Percivals' Lagrangian (see $[12,15]$ ), but this will not be needed.

Suppose $\omega$ is an irrational number. It follows easily from the order property (Proposition 2.7) of $M_{f, \omega}$ that $\phi_{\omega}$ is unique at points of continuity, up to composition on the right with a translation. More precisely, if $\phi^{\prime}$ is a second function satisfying the same conditions (1)-(3) (with respect to the same irrational number $\omega)$, then there exists $a \in \mathbb{R}$ such that

$$
\phi^{\prime}(t \pm)=\phi_{\omega}(t+a \pm) \text {. }
$$

In the case that $\omega$ is a rational number $p / q$, this still holds in the case that all periodic minimal configurations of type $(p, q)$ are translates of a single one, but not in general.

In order to state Proposition 6.1, we choose a real number $\omega$ and let $\phi_{\omega}$ be a function satisfying conditions (1)-(3) above. We let $x$ be one of $x_{+}^{\omega, t}$ or $x_{-}^{\omega, t}$, for some $t \in \mathbb{R}$, i.e., $x_{i}=\phi_{\omega}(t+\omega i \pm)$.

We also choose a real number $a$ such that $P_{\omega}(a)>0$ (assuming that such a number exists). For each integer $i$, we let $a_{i}$ be the unique real number such that $a_{i}-a$ is an integer and $x_{i} \in\left(a_{i}, a_{i}+1\right)$. Note that $x_{i}-a$ is not an integer, since $P_{\omega}(a)>0$.

Below, we will associate to certain pairs $(\omega, a)$ of real numbers an integer $K(\omega, a)$. For other such pairs, $K(\omega, a)$ will be undefined.

Proposition 6.1. Let $\mathcal{J}=\left(\ldots, J_{i}, \ldots\right)$ be a constraint. Let $j_{0} \leq j_{1}$ be integers, and let $\omega, a$ be real numbers. Suppose that $K(\omega, a)$ is defined and $J_{i}=$ $\left[a_{i}, a_{i}+1\right]$ for $j_{0}-K(\omega, a) \leq i \leq j_{1}+K(\omega, a)$, with $a_{i}$ defined as above. Let $\xi=\left(\ldots, \xi_{i}, \ldots\right)$ be a $\mathscr{J}$-minimal configuration. Then

$$
a_{i}<\xi_{i}<a_{i}+1, \text { for } j_{0} \leq i \leq j_{1} .
$$

We will prove several other propositions of the same general form: We define a constraint $\mathcal{J}$ explicitly over a range of indices in terms of a minimal configuration (in this case $x$ ). We then prove that a $\mathscr{J}$-minimal configuration is free over a smaller range of indices.

In order to complete the statement of Proposition 6.1, we now define an integer $K(\omega, a)$. If $P_{\omega}(a)=0$, then $K(\omega, a)$ will be undefined.

We let $p_{n} / q_{n}, n=0,1,2, \ldots$, be the convergents of the continued fraction expansion of $\omega$. For the moment all we will need to know about convergents is: first, $1=q_{0} \leq q_{1}<q_{2}<q_{3}<\cdots$, and second, a positive integer $q$ is one of the $q_{n}$ 's if and only if $\left\|q^{\prime} \omega\right\|>\|q \omega\|$ for $q^{\prime}=1, \ldots, q-1$, where $\|\lambda\|$ is defined to be $\min \{|\lambda-n|: n \in \mathbb{Z}\}$. Moreover, $p_{n}$ is the nearest integer to $q_{n} \omega$ for $n \geq 1$. If $\omega$ is irrational, it has infinitely many convergents, whereas if $\omega=p / q$, the continued fraction expansion stops after finitely many steps and $p / q$ is the last convergent.

The definition of $K(\omega, a)$ depends on a choice of $\beta$ such that $\bar{f} \in \mathscr{P}_{\beta}^{1}$. We choose one such $\beta$ and keep it fixed throughout the rest of this paper. We set $\theta=\cot \beta$, a notation that we will also retain throughout the rest of this paper. 
If $P_{\omega}(a)>0$, we let $k$ be the smallest integer such that $k>2 \theta / P_{\omega}(a)$. We let $n$ be the smallest integer such that $k<q_{n}$, if such an integer exists (i.e., if $\omega$ is irrational or $\omega=p / q$ in lowest terms with $k<q$ ). We set $K(\omega, a)=q_{n-1}+q_{n}$, if $n$ exists, and declare that $K(\omega, a)$ is undefined, otherwise.

This completes the definition of $K(\omega, a)$. Note that $K(\omega, a)$ actually depends on $\beta$ and Peierls's barrier as well, but we suppress these from the notation, since $\beta$ depends only on $f$ and $P_{\omega}(a)$ depends only on $f, \omega$, and $a$. Moreover, $f$ is held fixed throughout this paper.

For simplicity, we set $K=K(\omega, a)$ in the proof below.

Proof of Proposition 6.1. For simplicity, we suppose that $j_{0}=j_{1}$ and write $j$ for their common value. Obviously, this case implies the general form of Proposition 6.1. We will prove that $\xi_{j}<a_{j}+1$, the proof that $\xi_{j}>a_{j}$ being similar.

Let $s$ be the unique real number such that $\phi_{\omega}(s+\omega j-)<a_{j}+1<$ $\phi_{\omega}(s+\omega j+)$. Let $y_{i}=\phi_{\omega}(s+\omega i-)$. Thus $y$ (as well as $x$ ) is a minimal configuration whose rotation number is $\omega$. Obviously, $t \leq s$ and, consequently, $x \leq y$.

First, we give the proof in the case that there exist $i_{0}$ satisfying $j-K \leq i_{0}<j$ and $i_{1}$ satisfying $j<i_{1} \leq j+K$ such that $a_{i}+1<y_{i}$ for $i=i_{0}, i_{1}$. In this case, it is easy to see that $\xi_{i}<y_{i}$ for $i_{0} \leq i \leq i_{1}$ (and, in particular for $i=j$, which implies what we were to prove). The proof of this is practically identical with the proof of the Aubry crossing lemma, which states that the Aubry graphs of two minimal configurations cross at most once. (See Bangert [3, Lemma 3.1]: recall that the Aubry graph of a configuration $z=\left(\ldots, z_{i}, \ldots\right)$ is the broken curve in the plane that is the union of the line segments joining $\left(i, z_{i}\right)$ and $\left(i+1, z_{i+1}\right)$.)

We recall this proof in our context. We have

$$
\begin{aligned}
& h\left((\xi \wedge y)_{i(0)}, \ldots,(\xi \wedge y)_{i(1)}\right)+h\left((\xi \vee y)_{i(0)}, \ldots,(\xi \vee y)_{i(1)}\right) \\
& \quad \leq h\left(\xi_{i(0)}, \ldots, \xi_{i(1)}\right)+h\left(y_{i(0)}, \ldots, y_{i(1)}\right)
\end{aligned}
$$

by $\left(\mathrm{H}_{5}\right)$. Here $(\xi \wedge y)_{i}=\min \left(\xi_{i}, y_{i}\right),(\xi \vee y)_{i}=\max \left(\xi_{i}, y_{i}\right)$. Equality holds if and only if all crossings occur at nodes. If we do not have equality, we have a contradiction either to the assumption that $\xi$ is $\mathscr{J}$-minimal (since $\xi \wedge y$ is a $\mathcal{J}$-configuration that agrees with $\xi$ at $i=i_{0}, i_{1}$ ), or to the assumption that $y$ is minimal (since $\xi \vee y$ agrees with $y$ at $i=i_{0}, i_{1}$ ). If we have equality, this same argument shows that

$$
\begin{aligned}
& h\left((\xi \wedge y)_{i(0)}, \ldots,(\xi \wedge y)_{i(1)}\right)=h\left(\xi_{i(0)}, \ldots, \xi_{i(1)}\right), \\
& h\left((\xi \vee y)_{i(0)}, \ldots,(\xi \vee y)_{i(1)}\right)=h\left(y_{i(0)}, \ldots, y_{i(1)}\right) .
\end{aligned}
$$

But if the graphs cross at $i,(\xi \wedge y)$ or $(\xi \vee y)$ is not stationary, by $\left(\mathrm{H}_{5}\right)$ and $\left(\mathrm{H}_{6}\right)$, so we can slightly perturb one of them to get a contradiction to the minimality assumptions. 
This proves $\xi_{j}<y_{j}$ in the special case. Returning to the general situation, we set

$$
z_{i}=\phi_{\omega}\left(s+\left\|q_{n-1} \omega\right\|+\omega i+\right) .
$$

Thus, $z=\left(\ldots, z_{i}, \ldots\right)$ is a minimal configuration. Suppose $y_{i}<a_{i}+1$ for $j \leq i \leq j+K$. Note that $q_{n}$ is the smallest $q$ such that $\|q \omega\|<\left\|q_{n-1} \omega\right\|$. It follows that the projections in $\mathbb{R} / \mathbb{Z}$ of the intervals $[(i-j) \omega,(i-j) \omega+$ $\left.\left\|q_{n-1} \omega\right\|\right], i=j, \ldots, j+q_{n}-1$, do not overlap. Since $\phi_{\omega}$ is injective and $\phi_{\omega}(t+1)=\phi_{\omega}(t)+1$, this has the consequence that the projections in $\mathbb{R} / \mathbb{Z}$ of the intervals $\left[y_{i}, z_{i}\right], i=j, \ldots, j+q_{n}-1$, do not overlap. Since $k \leq q_{n}-1$, it follows that there exists $i_{1}$ satisfying $j<i_{1} \leq j+k$ such that $z_{i(1)}-y_{i(1)} \leq k^{-1}$. Moreover, $y_{j}<a_{j}+1<z_{j}$, by definition of $y_{j}$ and $z_{j}$. Since the projection in $\mathbb{R} / \mathbb{Z}$ of $\left[y_{i}, z_{i}\right]$ does not overlap that of $\left[y_{j}, z_{j}\right]$, for $j<i \leq i_{1}$, it follows that $a_{i}+1$ is not in $\left[y_{i}, z_{i}\right]$. Since $y_{i}<a_{i}+1$, we then obtain $z_{i}<a_{i}+1$, for $j<i \leq i_{1}$.

Let $i_{2}$ be the smallest value of $i>j$ for which [ $\left.(i-j) \omega,(i-j) \omega+\left\|q_{n-1} \omega\right\|\right]$ contains an integer. We have $i_{1}<i_{2} \leq j+K$; in fact, $i_{2}=j+q_{n}$ or $i_{2}=$ $j+q_{n}+q_{n-1}$, as follows easily from the fact that $q_{n}$ is the smallest integer $q$ such that $\|q \omega\|<\left\|q_{n-1} \omega\right\|$.

Now $z$ is a minimal configuration satisfying $a_{j}+1<z_{j}, a_{i(2)}+1<z_{i(2)}$ and $a_{i}<z_{i}<a_{i}+1$ for $j<i<i_{2}$. Since $\xi$ is $\mathscr{J}$-minimal, we obtain (just as before, using the Aubry crossing argument) that $\xi_{i}<z_{i}$ for $j \leq i \leq i_{2}$. In particular, $\xi_{i(1)}<z_{i(1)}<y_{i(1)}+k^{-1}$.

Thus, we have shown that there exists $j<i_{1} \leq j+K$ such that $\xi_{i(1)}<$ $y_{i(1)}+k^{-1}$ and $y_{i}<a_{i}+1$, for $j \leq i<i_{1}$. Similarly, there exists $j-K \leq i_{0}<j$ such that $\xi_{i(0)}<y_{i(0)}+k^{-1}$, and $y_{i}<a_{i}+1$, for $i_{0}<i \leq j$. (If there exists $j-K \leq i_{0}<j$ such that $a_{i(0)}+1<y_{i(0)}$, then $\xi_{i(0)}<y_{i(0)}$; otherwise, the argument we have just given applies.)

We will prove $\xi_{j}<a_{j}+1$, by contradiction. We set $w_{i}=\xi_{i}, i=i_{0}, i_{1}$; and $w_{i}=\min \left(y_{i}, \xi_{i}\right), i_{0}<i<i_{1}$. We will show that $h\left(w_{i_{0}}, \ldots, w_{i_{1}}\right)<$ $h\left(\xi_{i_{0}}, \ldots, \xi_{i_{1}}\right)$, if $\xi_{j}=a_{j}+1$. Since $\xi$ is $\mathscr{J}$-minimal, this will be the desired contradiction.

We set $v_{i}=y_{i}$, for $i=i_{0}, i_{1} ; \quad v_{i}=\max \left(\xi_{i}, y_{i}\right)$, for $i_{0}<i<i_{1} ; \widetilde{w}_{i}=$ $\min \left(y_{i}, \xi_{i}\right) ; \widetilde{v}_{i}=\max \left(y_{i}, \xi_{i}\right)$. Note that $v_{i}$ and $\widetilde{v}_{i}$ are the same, except at the endpoints $i=i_{0}$ and $i=i_{1}$, and there they differ by at most $k^{-1}$. The same remark holds for $w_{i}$ and $\widetilde{w}_{i}$.

To simplify the notation, we will write $h(w)$ for $h\left(w_{i_{0}}, \ldots, w_{i_{1}}\right)$, etc., in the rest of this proof. We have

$$
h(w)-h(\widetilde{w})+h(v)-h(\widetilde{v}) \leq 2 \theta k^{-1} .
$$

Since $\widetilde{v}$ agrees with $v$ and $\widetilde{w}$ agrees with $w$ except at the ends of the interval $\left[i_{0}, i_{1}\right]$, it is clear that the left side is the sum of two terms, one coming from each end of the interval $\left[i_{0}, i_{1}\right]$. If $\xi_{i_{0}} \leq y_{i_{0}}$, the term coming from the $i_{0}$ end 
vanishes, and otherwise it is

$$
\begin{aligned}
h\left(\xi_{i_{0}},\right. & \left.w_{i_{0}+1}\right)-h\left(y_{i_{0}}, w_{i_{0}+1}\right)+h\left(y_{i_{0}}, v_{i_{0}+1}\right)-h\left(\xi_{i_{0}}, v_{i_{0}+1}\right) \\
= & \int_{y_{i_{0}}}^{\xi_{i_{0}}}\left(\partial_{1} h\left(\alpha+, w_{i_{0}+1}\right)-\partial_{1} h\left(\alpha+, v_{i_{0}+1}\right)\right) d \alpha \leq \theta k^{-1},
\end{aligned}
$$

since the integral is taken over the interval $\left[y_{i_{0}}, \xi_{i_{0}}\right]$, which has length $\leq k^{-1}$, and the quantity under the integral sign is $\leq \theta$. For, when $\beta \leq \gamma \leq \beta+1$, we have $\partial_{1} h(\alpha+, \beta) \leq \partial_{1} h(\alpha-1+, \beta)+\theta=\partial_{1} h(\alpha+, \beta+1)+\theta \leq \partial_{1} h(\alpha+, \gamma)+\theta$ by $\left(\mathbf{H}_{6 \theta}\right),\left(\mathbf{H}_{6}\right)$, and $\left(\mathbf{H}_{5}\right)$. Note that $a_{i_{0}+1} \leq v_{i_{0}+1} \leq w_{i_{0}+1} \leq a_{i_{0}+1}+1$, so we may apply this remark with $\beta=v_{i_{0}+1}$, and $\gamma=w_{i_{0}+1}$. This proves the inequality above. The previous one is a consequence of it and the corresponding inequality coming from the $i_{1}$ end.

Thus, $h(w) \leq h(\widetilde{w})+h(\widetilde{v})-h(v)+2 \theta k^{-1} \leq h(y)+h(\xi)-h(v)+2 \theta k^{-1} \leq$ $h(\xi)-P_{\omega}(a)+2 \theta k^{-1}<h(\xi)$. We just derived the first inequality. The second inequality is a consequence of the definitions $\widetilde{w}=y \wedge \xi, \widetilde{v}=y \vee \xi$, and the condition $\left(\mathrm{H}_{5}\right)$. For the third inequality, we use the assumption that $\xi_{j}=a_{j}+1$. Then $v_{j}=a_{j}+1$. Since $v_{i}=y_{i}$ for $i=i_{0}, i_{1}$ and $y$ is the minimal configuration of rotation symbol $\omega$ defined by $y_{i}=\phi_{\omega}(s+\omega i-)$, where $\phi_{\omega}(s+\omega j-)<a_{j}+1<\phi_{\omega}(s+\omega j+)$, it follows that $h(v)-h(y) \geq P_{\omega}\left(a_{j}+1\right)=$ $P_{\omega}(a)$, which is the third inequality. We chose $k$ so that $k>2 \theta / P_{\omega}(a)$, which is the fourth inequality.

To summarize, assuming $\xi_{j}=a_{j}+1$, we have proved the inequality $h(w)<$ $h(\xi)$. This inequality contradicts the fact that $\xi$ is $\mathscr{J}$-minimal. By this contradiction, we conclude that $\xi_{j}<a_{j}+1$.

In Proposition 6.1, the barriers $a_{i}$ all differ from $a$ by an integer, so one might say that the proof of $\mathscr{J}$-freeness is based on the use of a single barrier $a$. At times, we will need a slightly more flexible result, where two barriers are permitted.

This result may be formulated as follows: Consider real numbers $\omega, a$, and $b$, such that $P_{\omega}(a)>0$ and $P_{\omega}(b)>0$. As before, we let $p_{n} / q_{n}$ denote the convergents of $\omega$. We let $k$ be the smallest integer such that $k^{-1}<$ $\min \left(P_{\omega}(a) / 2 \theta, P_{\omega}(b) / 2 \theta\right)$. We let $n$ be the smallest integer such that $k<q_{n}$, if such an integer exists. We set $K=k+q_{n-1}+q_{n}$ if $n$ exists, and say that $K$ is undefined, otherwise. We choose a real number $t$ and let

$$
x=x_{ \pm}^{\omega, t}=\left(\ldots, \phi_{\omega}(t+\omega i \pm), \ldots\right) .
$$

We consider an integer $j^{*}$. For $i<j^{*}$, we let $a_{i}$ be the unique real number such that $a_{i}-a$ is an integer and $x_{i} \in\left(a_{i}, a_{i}+1\right)$. For $i \geq j^{*}$, we let $a_{i}$ be the unique real number such that $a_{i}-b$ is an integer and $x_{i} \in\left(a_{i}, a_{i}+1\right)$.

Proposition 6.2. Let $\mathscr{J}=\left(\ldots, J_{i}, \ldots\right)$ be a constraint. Suppose that $K$ is defined and $J_{i}=\left[a_{i}, a_{i}+1\right]$ for $j_{0}-K \leq i \leq j_{1}+K$, for some integers $j_{0}<j^{*} \leq j_{1}$. Then we have the same conclusion as before. 
The main difference between this and Proposition 6.1 is the change in the definition of $a_{i}$ : we change barriers at $j^{*}$.

Proof. We indicate only the modifications of the previous proof. We consider a $\mathcal{J}$-minimal configuration $\xi$ and an integer $j$ satisfying $j_{0} \leq j \leq j_{1}$. We will prove $\xi_{j}<a_{j}+1$, the proof of $a_{j}<\xi_{j}$ being similar.

We define minimal configurations $y$ and $z$ exactly as before. We will find integers $i_{0}, i_{1}$ satisfying $j_{0}-K \leq i_{0}<j<i_{1} \leq j_{1}+K$ such that

$$
\xi_{i} \leq y_{i}+k^{-1}, \quad \text { for } i=i_{0}, i_{1},
$$

and $y_{i}<a_{i}+1$, for $i_{0}<i<i_{1}$. Once such integers have been found, the proof proceeds exactly as before.

To find $i_{1}$, we proceed as follows. If there is an $i$ such that $j<i \leq j_{1}+K$ and $a_{i}+1<y_{i}$, we let $i_{1}$ be the least such $i$. Then $\xi_{i_{1}}<a_{i_{1}}+1<y_{i_{1}}$, which is more than required. Otherwise, there exists $j<i_{1} \leq j+k$ such that $z_{i_{1}}-y_{i_{1}}<k^{-1}<\varepsilon$ (for the same reason as before), and $i_{1}<i_{2} \leq j_{1}+K$ such that $a_{i_{2}}+1<z_{i_{2}}$. Here the reason is essentially the same as before, but we must use the new definition of $K$ for the argument to work. The proof in this case is based on the observation that the projections on $\mathbb{R} / \mathbb{Z}$ of the intervals $\left[(i-j) \omega,(i-j) \omega+\left\|q_{n-1} \omega\right\|\right], i=j_{1}+k+1, \ldots, j_{1}+K$, cover $\mathbb{R} / \mathbb{Z}$. (Since we have two barriers, it is not enough that one of these intervals contains an integer in the case $j<j^{*}$.) Otherwise, the argument works exactly as before.

\section{SOME $\mathscr{J}$-FREE CONFIGURATIONS}

In the previous section, we showed that if $\mathscr{J}=\left(\ldots, J_{i}, \ldots\right)$ is a constraint, $J_{i}$ has a special form in a certain range of indices $j_{0}-K \leq i \leq j_{1}+K$, and $\xi=\left(\ldots, \xi_{i}, \ldots\right)$ is a $\mathscr{J}$-minimal configuration, then $\xi_{i} \in \operatorname{int} J_{i}$, for $i$ in a smaller range of indices $j_{0} \leq i \leq j_{1}$. In Proposition 6.1, we had $J_{i}=\left[a_{i}, a_{1}+1\right]$ for $j_{0}-K \leq i \leq j_{1}+K$, where $a_{i} \equiv a(\bmod 1)$ and $x_{i} \in\left(a_{i}, a_{i}+1\right)$ in that range, $x=\left(\ldots, x_{i}, \ldots\right)$ being a ground configuration (a minimal configuration whose corresponding orbit is recurrent).

Thus, knowing the ground configuration $x$ and the number $a$ uniquely specifies the $J_{i}$ 's in the range $j_{0}-K \leq i \leq j_{1}+K$. One may say that the $J_{i}$ 's "follow" the ground configuration $x$ in that range. Obviously, there are other ground configurations $y$ that these $J_{i}$ 's also follow. There is, in fact, often an open interval $\Omega$ such that for every $\omega \in \Omega$, there is a ground configuration $y$ of rotation number $\omega$ such that the $J_{i}$ 's follow $y$ in the range $j_{0}-K \leq i \leq j_{i}+K$.

Next, we consider how we may construct very long sequences of $J_{i}$ 's such that in relatively short subsequences Proposition 6.1 applies. "Relatively short" does not mean "short" since for Proposition 6.1 to apply, the length of the "relatively short subsequences" has to be greater than $2 K$.

Let $n=\left(\ldots, n_{i}, \ldots\right)$ be a bi-infinite sequence of integers, and let $\varepsilon>0$. We will say that $n$ is $\varepsilon$-restrained, if for each $j \in \mathbb{Z}$, there exist real numbers 
$\omega_{j}$ and $s_{j}$ such that the following hold. Let $p_{j 1} / q_{j 1}, p_{j 2} / q_{j 2}, \ldots$ be the convergents of the continued fraction expansion of $\omega_{j}$. We suppose that $\omega_{j}$ is irrational or rational with denominator (in lowest terms) $>\varepsilon^{-1}$. We let $l_{j}$ be the smallest integer such that $q_{j l(j)}>\varepsilon^{-1}$. We let $K_{j}=q_{j, l(j)-1}+q_{j, l(j)}$. We require that $n_{i}<\omega_{j} i+s_{j}<n_{i}+1$, for $j-K_{j} \leq i \leq j+K_{j}$.

Let $\Omega$ be an open interval in $\mathbb{R}$. If $n$ is $\varepsilon$-restrained and, in addition, $\omega_{j} \in \Omega$, for each $j \in \mathbb{Z}$, we say that $n$ is $(\varepsilon, \Omega)$-restrained.

Proposition 7.1. Let $n=\left(\ldots, n_{i}, \ldots\right) \in \mathbb{Z}^{\mathbb{Z}}$. Let $\varepsilon=k^{-1}$, where $k$ is a positive integer. Let $\Omega$ be an open interval. Let $a \in \mathbb{R}$. Suppose that for all $\omega \in \Omega$,

$$
P_{\omega}(a)>2 \theta / k \text {. }
$$

Suppose that $n$ is $\left(k^{-1}, \Omega\right)$-restrained. Let $a_{i}=a+n_{i}, J_{i}=\left[a_{i}, a_{i+1}\right]$, and $\mathscr{J}=\left(\ldots, J_{i}, \ldots\right)$. Then every $\mathscr{J}$-minimal configuration is $\mathcal{J}$-free and therefore corresponds to an orbit of $\bar{f}$.

Proof. This is simply a matter of checking that the hypotheses of Proposition 6.1 are satisfied, for every $j \in \mathbb{Z}$. Let $s$ be the unique real number such that $\phi_{\omega}(s-)<a<\phi_{\omega}(s+)$, where $\omega=\omega_{j}$. Let $x_{i}^{(j)}=\phi_{\omega}\left(s+s_{j}+\omega_{j} i-\right)$ and let $x^{(j)}=\left(\ldots, x_{i}^{(j)}, \ldots\right)$. Then $a_{i}<x_{i}^{(j)}<a_{i+1}$ for $j_{0}-K_{j} \leq i \leq j_{1}+K_{j}$, since $n_{i}<\omega_{j} i+s_{j}<n_{i}+1$, in the same range. Note that $K_{j}$ is defined in terms of $\omega_{j}$ and $k$ in the same way as $K=K(\omega, a)$ was defined in terms of $\omega$ and $k$ in Proposition 6.1. The only restriction on $k$ in Proposition 6.1 is $k>2 \theta / P_{\omega}(a)$, which is satisfied by $\varepsilon$ here by hypothesis.

The advantage of Proposition 7.1 is that it reduces the problem of finding constraints $\mathscr{J}=\left(\ldots, J_{i}, \ldots\right)$ such that $\mathscr{J}$-minimal implies $\mathscr{J}$-free to the purely number-theoretic problem of finding $\varepsilon$-restrained bi-infinite sequences of integers. The limitation of Proposition 7.1 is that the class of constraints $\mathcal{J}$ that it provides is not nearly sufficient for our purposes. This limitation of Proposition 7.1 will provide the motivation for Proposition 8.1.

Next, we discuss sufficient conditions for $n \in \mathbb{Z}^{\mathbb{Z}}$ to be $\varepsilon$-restrained. For $j<k \in \mathbb{Z}$, we let $A_{n}[j, k]=A[j, k]$ denote the open interval in $\mathbb{R}$ whose endpoints are $\left(n_{k}-n_{j}-1\right) /(k-j)$ and $\left(n_{k}-n_{j}+1\right) /(k-j)$. We let $B_{n}[j, k]=$ $B[j, k]$ denote the intersection of all $A\left[j^{\prime}, k^{\prime}\right]$, for $j \leq j^{\prime}<k^{\prime} \leq k$. Thus, $B[j, k]$ is an open interval or is void.

Lemma 7.2. If $\omega \in B[j, k]$, there exists $s \in \mathbb{R}$ such that

$$
n_{i}<\omega i+s<n_{i}+1, \quad \text { for } j \leq i \leq k .
$$

Proof. Obviously, $\omega \in A\left[j^{\prime}, k^{\prime}\right]$ if and only if the two open intervals $\left(n_{j^{\prime}}-\right.$ $\left.j^{\prime} \omega, n_{j^{\prime}}-j^{\prime} \omega+1\right)$ and $\left(n_{k^{\prime}}-k^{\prime} \omega, n_{k^{\prime}}-k^{\prime} \omega+1\right)$ have nonvoid intersection, since the endpoints of $A\left[j^{\prime}, k^{\prime}\right]$ are the minimum and maximum slopes of line segments joining $\left[n_{j^{\prime}}, n_{j^{\prime}}+1\right]$ and $\left[n_{k^{\prime}}, n_{k^{\prime}}+1\right]$. The hypothesis that 
$\omega \in B[j, k]$ therefore implies that any two of the intervals $\left(n_{i}-i \omega, n_{i}-i \omega+1\right)$, $j \leq i \leq k$, have nonvoid intersection. It follows easily that the family of all these intervals (for $j \leq i \leq k$ ) has nonvoid intersection. A point $s$ in the intersection satisfies the conclusion of the lemma.

For what we do next we indicate the dependence of $K$ on $\omega$ and $\varepsilon$ explicitly. Let us recall the definition. Let $p_{1} / q_{1}, p_{2} / q_{2}, \ldots$ be the convergents of the continued fraction of $\omega$. Let $l$ be the smallest integer such that $g_{l}>\varepsilon^{-1}$. If there is no such $l$, we leave $K$ undefined. When there is such an $l$, we set $K=K(\omega)=K(\omega, \varepsilon)=q_{l-1}+q_{l}$.

Lemma 7.3. Let $n \in \mathbb{Z}^{\mathbb{Z}}$. Then $n$ is e-restrained if for each $j \in \mathbb{Z}$ there exists $\omega=\omega_{j}$ such that $K(\omega, \varepsilon)$ is defined and

$$
\omega \in B_{n}[j-K(\omega, \varepsilon), j+K(\omega, \varepsilon)] .
$$

Proof. Immediate from Lemma 7.2 and the definition of what it means for $n$ to be $\varepsilon$-restrained.

Note that $K(\omega, \varepsilon)$ is undefined if $\omega$ is a rational number $p / q$ with $q \leq \varepsilon^{-1}$. In all other cases, $K(\omega, \varepsilon)$ is defined.

We may estimate the size of $K(\omega, \varepsilon)$, as follows. Let $F\left(\varepsilon^{-1}\right)$ denote the set of rational numbers whose expression $p / q$ in lowest form satisfies $q \leq \varepsilon^{-1}$. Let $\Delta_{\varepsilon}(\omega)=\min \left\{|q \omega-p|: p / q \in F\left(\varepsilon^{-1}\right)\right\}$. We have

$$
1 / 2 \Delta_{\varepsilon}(\omega) \leq K(\omega, \varepsilon) \leq 2 / \Delta_{\varepsilon}(\omega),
$$

and $K(\omega, \varepsilon)$ is undefined if and only if $\Delta_{\varepsilon}(\omega)=0$. This estimate follows from basic facts concerning the convergents $p_{1} / q_{1}, \ldots$ of the continued fraction expansion of $\omega$. With $l$ as in the definition of $K$, we have

$$
1 / 2 q_{l} \leq\left|q_{l-1} \omega-p_{l-1}\right| \leq 1 / q_{l},
$$

as is true of any two successive convergents of the continued fraction expansion of any real number $\omega$. The estimate above on $K(\omega, \varepsilon)$ follows immediately, in view of $K(\omega, \varepsilon)=q_{l}+q_{l-1}$ and $\Delta_{\varepsilon}(\omega)=\left|q_{l-1} \omega-p_{l-1}\right|$.

If $M$ is a real number, we let $F(M)$ denote the set of all rational numbers whose denominator (in lowest terms) is less than or equal to $M$. We will call a component of the complement of $F(M)$ an open Farey interval of height $M$, in analogy to the notion of "Farey series" in elementary number theory. We call the closure of such an interval a closed Farey interval of height $M$. If $n=\left(\ldots, n_{i}, \ldots\right)$ is an $\varepsilon$-restrained bi-infinite sequence of integers, an open Farey interval $\left(p / q, p^{\prime} / q^{\prime}\right)$ of height $\varepsilon^{-1}$ will be said to be the Farey interval associated to $n$ if $A_{n}[j, k]$ intersects $\left(p / q, p^{\prime} / q^{\prime}\right)$ for all $j<k$. It is not difficult to see that every $\varepsilon$-restrained bi-infinite sequence of integers has a unique Farey interval (of height $\varepsilon^{-1}$ ) associated to it. However, we will not need this result, and will not prove it.

What we need is a sort of converse: that it is possible to construct $\varepsilon$-restrained sequences that permit us to achieve arbitrary sequences of rotation numbers, as 
long as we stay within one Farey interval of height $\varepsilon^{-1}$. For this purpose, we consider a Farey interval $\left(p / q, p^{\prime} / q^{\prime}\right)$ of height $\varepsilon^{-1}$ and a closed subinterval $\Omega$ of $\left(p / q, p^{\prime} / q^{\prime}\right)$. In view of the estimate above on $K(\omega, \varepsilon)$, there exists $K$ such that $K(\omega, \varepsilon) \leq K$ for $\omega \in \Omega$. Simple examples of $\varepsilon$-restrained sequences $n=\left(\ldots, n_{i}, \ldots\right)$ may be obtained of the form $n_{i}=\left[\lambda_{i}\right]$ (i.e., $n_{i}=$ the greatest integer $\leq \lambda_{i}$ ), where

$$
\lambda_{i+1}-\lambda_{i}=r_{j}, \quad j K \leq i<(j+1) K,
$$

$r_{j}$ is a rational number of denominator $\leq 2 K$ in $\Omega$, and $\left[r_{j}, r_{j+1}\right]$ is a Farey interval of height $2 K$, for each $j$. In this case, Lemma 7.3 applies with $\omega_{i}=r_{j}$ for $j K \leq i<(j+1) K$. It follows that $n$ is $\varepsilon$-restrained.

Now we can state weak versions of Theorems 4.1 and 4.2. These will show how far we can get towards a proof of Theorems 4.1 and 4.2 using only Proposition 6.1 and provide motivation for Proposition 8.1.

In Propositions 7.4 and 7.5, we consider $a \in \mathbb{R}, P>0$, and an open interval $\Omega \subset \mathbb{R}$. We let $k$ be the smallest integer $>2 \theta / P$ and suppose that for every rational number $p / q$ in $\Omega$, we have $q>k$. We suppose that for every $\omega \in \Omega$, $P_{\omega}(a)>P$.

Proposition 7.4. Consider $\omega_{-}, \omega_{+} \in \Omega$. There exists an $\bar{f}$-orbit $\mathscr{O}$ that is $\alpha$-asymptotic to $M_{\bar{f}, \omega_{-}}$and $\omega$-asymptotic to $M_{\bar{f}, \omega_{+}}$.

Proposition 7.5. Consider $\omega_{i} \in \Omega$ and $\varepsilon_{i}>0$ for every integer $i$. There exists an $\bar{f}$-orbit $\mathscr{O}=\left(\ldots,\left(\theta_{i}, y_{i}\right), \ldots\right)$ and an increasing sequence $\left(\ldots, j_{i}, \ldots\right)$ of integers such that

for every $i \in \mathbb{Z}$.

$$
\operatorname{dist}\left(\left(\theta_{j(i)}, y_{j(i)}\right), M_{\bar{f}, \omega(i)}\right)<\varepsilon_{i}
$$

Proof. Let $\omega^{*} \in \Omega$ and $\varepsilon^{*}>0$. Let $x^{*}=\left(\ldots, x_{i}^{*}, \ldots\right)$ be a minimal configuration of rotation symbol $\omega^{*}$. Let $a_{i}^{*}$ be the unique number such that $a_{i}^{*}-a$ is an integer and $x_{i} \in\left(a_{i}^{*}, a_{i}^{*}+1\right)$. We consider a constraint $\mathscr{J}=\left(\ldots, J_{i}, \ldots\right)$ such that $J_{i}=\left[a_{i}^{*}, a_{i}^{*}+1\right]$ over a certain range $i_{-}^{*} \leq i \leq i_{+}^{*}$ of integers. We suppose that every $\mathscr{J}$-minimal configuration is free. Let $\mathscr{O}=\left(\ldots,\left(\theta_{i}, y_{i}\right), \ldots\right)$ be an $\bar{f}$-orbit corresponding to a $\mathscr{J}$-minimal configuration. In $\S 10$, we show that there is a number $K^{*}$ such that $\operatorname{dist}\left(\left(\theta_{i}, y_{i}\right): M_{\bar{f}, \omega^{*}}\right)<\varepsilon^{*}$ for $i_{-}^{*}-K^{*} \leq$ $i \leq i_{+}^{*}+K^{*}$. This number depends on $\bar{f}, \omega^{*}$, and $\varepsilon^{*}$, but not on $i_{-}^{*}, i_{+}^{*}$.

Thus, Propositions 7.4 and 7.5 reduce to constructing constraints with certain properties. By Proposition 7.1 , these reduce to constructing certain $\left(k^{-1}, \Omega\right)$ restrained bi-infinite sequences of integers. Appropriate sequences of this type may be constructed by the discussion preceding the statement of Propositions 7.4 and 7.5 .

\section{More partially $\mathscr{J}$-Free configurations}

In Propositions 7.4 and 7.5, we have shown how to construct orbits that approach other orbits whose rotation numbers lie in an interval $\Omega$. The limitation 
of these results is that the interval $\Omega$ cannot contain any rational numbers $p / q$ with $q \leq 2 \theta / P$, where $P=\sup _{a \in \mathbb{R}} \inf _{\omega \in \Omega}\left\{P_{\omega}(a)\right\}$. If $\Omega$ contains a rational number with such a small denominator, then Propositions 7.4 and 7.5 do not apply. These two propositions were deduced from Proposition 6.1, which does not apply in this circumstance either.

In this section, we state and prove an analogue of Proposition 6.1 that allows us to overcome this limitation.

Let $p / q$ be a rational number, expressed in lowest terms, with $q \geq 0$. In this section, we will show (loosely speaking) how to construct a segment of an orbit that first follows a minimal orbit of rotation number slightly less than (resp. greater than) $p / q$, then follows a minimal orbit of rotation symbol $p / q-$ (resp. $p / q+$ ), then follows a minimal orbit of rotation symbol $p / q+$ (resp. $p / q-$ ), and finally follows a minimal orbit of rotation symbol slightly greater than (resp. less than) $p / q$. What we mean by "follows" is contained in the statement of Proposition 8.1, which is the analogue of Proposition 6.1 referred to above. Here, we use the word "follows" in a heuristic sense only, since it would not be useful to give a precise definition. In a similar vein, we may say that Proposition 6.1 provides a segment of an orbit that follows a given minimal orbit of irrational rotation number or rational rotation number of large denominator.

We may reduce the problem we consider in this section to the case that $p / q=0$, by the following device: Let $F(x, y)=f^{q}(x, y)-(p, 0)$. If $\left(\ldots,\left(x_{i}, y_{i}\right), \ldots\right)$ is an orbit of $f$, then for any $j \in \mathbb{Z}$, we have that $\left(\ldots,\left(x_{q i+j}-p i, y_{q i+j}\right), \ldots\right)$ is an orbit of $F$, and for each $j \in \mathbb{Z}$, this sets up a bijective correspondence between orbits of $f$ and orbits of $F$. Under any one of these correspondences, orbits of $f$ of rotation number $p / q$ correspond to orbits of $F$ of rotation number 0 .

Orbits of $F$ may be treated within the framework of this paper, since there is a variational principle $H$ for $F$ that satisfies the conditions $(\mathrm{H})$ : We let $*$ denote the conjunction operation, discussed in $\S 1$. We set $h^{* 2}=h * h$ and generally, $h^{*(n+1)}=h^{* n} * h$. We set

$$
H\left(x, x^{\prime}\right)=h^{* q}\left(x, x^{\prime}-p\right)-C,
$$

where the constant $C$ is given by

$$
C=\min _{x \in \mathbb{R}} h^{* q}(x, x-p) .
$$

In $\S 1$, we reviewed results of [16], which imply that $H$ is the variational principle of $F$. In fact, the variational principle of $F$ is uniquely defined up to addition of an arbitrary constant. (However, we have not proved this uniqueness result, and we will not use it.) Here, we have made an explicit choice of the arbitrary constant, so as to have

$$
\min _{x \in \mathbb{R}} H(x, x)=0 \text {. }
$$


Choosing the arbitrary constant so that $H$ satisfies this equation permits a considerable notational simplification in the proof of Proposition 8.1.

Before stating Proposition 8.1, we recall the following facts and notations: $M_{f, p / q}=M_{F, 0} \subset \mathbb{R}^{2}$ is the union of all minimal orbits of $f$ that are periodic of type $(p, q)$. It is also the set of fixed points of $F$. Letting $\pi: \mathbb{R}^{2} \rightarrow \mathbb{R}$ denote the projection on the first factor, we have that $\pi \mid M_{F, 0}: M_{F, 0} \rightarrow \mathbb{R}$ is proper and injective. In particular, $\pi\left(M_{F, 0}\right)$ is a closed subset of $\mathbb{R}$. Using a superscript to explicitly indicate the dependence of Peierls's barrier on $h$, we have $P_{p / q( \pm)}^{h}=P_{0( \pm)}^{H}$. If $\left(\ldots, x_{i}, \ldots\right)$ is a minimal configuration for $h$, then $\left(\ldots, x_{q i+j}-p i, \ldots\right)$ is a minimal configuration for $H$. For each $j \in \mathbb{Z}$, this sets up a bijective correspondence between minimal configurations for $h$ and minimal configurations for $H$.

Proposition 8.1. Let $\left(c, c^{\prime}\right)$ be a complementary interval of $\pi\left(M_{F, 0}\right)$. Let $a, b \in\left(c, c^{\prime}\right)$ and suppose that $P_{0-}^{H}(a)>0$ and $P_{0+}^{H}(b)>0$. Then there exist nonnegative integers $K_{-}, K_{+}, K, L_{-}^{0}$, and $L_{+}^{0}$ such that the following hold: Let $L_{-}, L_{+}, l_{-}, l_{+}, i_{-}, i_{0}$, and $i_{+}$be integers satisfying $L_{-} \geq L_{i}^{0}$, $L_{+} \geq L_{+}^{0}, l_{-} \geq 1, l_{+} \geq 1, i_{-}<i_{0}<i_{+}, i_{0}-i_{-}>K_{-}, i_{+}-i_{0}>K_{+}$, and $i_{+}-i_{-}>K+K_{-}+K_{+}$.

Let $\mathscr{J}=\left(\ldots, J_{i}, \ldots\right)$ be a constraint with $J_{i}$ defined for $i_{-}-L_{-} l_{-}-1 \leq$ $i \leq i_{+}+L_{+} l_{+}$in one of the following two ways:

$$
\begin{aligned}
J_{i}= & {[a+\beta-1, a+\beta] \quad(\text { resp. }[b-\beta, b-\beta+1]), } \\
& \text { if } i_{-}-L_{-} \beta \leq i<i_{-}-L_{-} \beta+L_{-} \text {and } 1 \leq \beta \leq l_{-}, \\
& \text {or } \beta=l_{-}+1 \text { and } i=i_{-}-L_{-} l_{-}-1, \\
J_{i}= & {[c, a] \quad\left(\text { resp. }\left[b, c^{\prime}\right]\right), \quad \text { if } i_{-} \leq i<i_{0}, } \\
J_{i}= & {[c, b] \quad\left(\text { resp. }\left[a, c^{\prime}\right]\right), \quad \text { if } i_{0} \leq i<i_{+}, } \\
J_{i}= & {[b+\beta-1, b+\beta] \quad(\text { resp. }[a-\beta, a-\beta+1]), } \\
& \text { if } i_{+}+L_{+} \beta-L_{+} \leq i<i_{+}+L_{+} \beta \text { and } 1 \leq \beta \leq l_{+}, \\
& \text {or } \beta=l_{+}+1 \text { and } i=i_{+}+L_{+} \beta .
\end{aligned}
$$

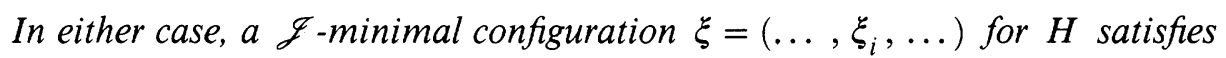
the condition that $\xi_{i} \in \operatorname{int} J_{i}$ for $i_{-}-L_{-} l_{-}+1 \leq i<i_{+}+L_{+} l_{+}-1$.

The positive integers $K_{-}, K_{+}, K, L_{-}^{0}$, and $L_{+}^{0}$ depend only on $\theta, P_{0_{+}}^{H}(b)$, $P_{0-}^{H}(a)$, and the function $H$. Explicit (but complicated) determinations of these numbers will be given in the proof.

In the proof, we will repeatedly use the formula

$$
\sum_{i=j}^{k-1} H\left(x_{i}, x_{i+1}\right)=\sum_{i=j}^{k-1} H\left(x_{i}, x_{i}\right)+\int_{x_{j}}^{x_{k}} \partial_{2} H(y, y+) d y+\sum_{i=j}^{k-1} \mu_{H}\left(\Delta_{i}\right),
$$


where $\Delta_{i}$ is the triangle

$$
\left\{(y, z): x_{i} \leq y \leq z \leq x_{i+1}\right\} \text { or }\left\{(y, z): x_{i+1} \leq z \leq y \leq x_{i}\right\},
$$

according to whether $x_{i}$ or $x_{i+1}$ is greater. Here, $\mu_{H}$ is the unique Borel measure on $\mathbb{R}^{2}$ that satisfies

$$
\mu_{H}\left([x, \xi] \times\left[x^{\prime}, \xi^{\prime}\right]\right)=H\left(\xi, x^{\prime}\right)+H\left(x, \xi^{\prime}\right)-H\left(x, x^{\prime}\right)-H\left(\xi, \xi^{\prime}\right),
$$

for any $x<\xi$ and $x^{\prime}<\xi^{\prime}$. The existence and uniqueness of $\mu_{H}$ is the content of the lemma in $[17, \S 3]$. Also, $\partial_{2} H(y, y+)$ is the one-sided first partial derivative of $H$ with respect to the second variable. We could just as well use $\partial_{2} H(y, y-)$ in formula (8.1), since $\partial_{2} H(y, y+)$ differs from $\partial_{2} H(y, y-)$ at most countably many points. The derivation of equation (8.2) is an elementary exercise, which was carried out in [17, 3.4]. Equation (8.2) is valid for any sequence $\left(x_{j}, \ldots, x_{k}\right)$ of real numbers.

We will prove Proposition 8.1 only for the case when $J_{i}=[c, a]$, if $i_{-} \leq$ $i<i_{0}$, etc. Obviously, the other case may be proved in the same way.

The proof is based on a sequence of lemmas.

Lemma a. Let $\hat{i}$ be a value of $i$ satisfying $i_{-} \leq i<i_{+}$for which $\xi_{i}$ takes its minimum value. Then the sequence $\left(\xi_{i}\right)$ is weakly monotone decreasing for $i_{-}-L_{-} l_{-}+L_{-}-1 \leq i \leq \hat{i}$ and weakly monotone increasing for $\hat{i} \leq i \leq$ $i_{+}+L_{+} l_{+}-L_{+}$.

Proof. If one of these sequences was not weakly monotone, then one could change it to make it monotone by reordering the indices. This could be done without changing the end terms. The new sequence would still be a segment of a $\mathscr{J}$-configuration. However, equation (8.2) shows that $\sum H\left(\xi_{i}, \xi_{i+1}\right)$, summed over the appropriate range, would be smaller for the new sequence than for the original sequence. This would contradict the assumption that the original sequence is a $\mathcal{J}$-minimal configuration.

We set $L_{-}^{0}=2\left[\varepsilon_{-}^{-1}\right]+1$, where $\varepsilon_{-}$is the unique positive solution of the equation $2 \varepsilon_{-}+\varepsilon_{-}^{2}=P_{0-}^{H}(a) / 8 \theta$.

Lemma b. If $L_{-} \geq L_{-}^{0}$ then $\xi_{i} \neq a+\beta$ when $i=i_{-}-L_{-} \beta-1$ or $i=i_{-}-L_{-} \beta$ and $\beta$ is an integer satisfying $1 \leq \beta \leq l_{-}-1$.

Proof. We suppose otherwise and show that there is a sequence $\eta_{i}$ with $i$ running from $i_{-}-L_{-} \beta-L_{-}-1$ to $i_{-}-L_{-} \beta+L_{-}$, with $\eta_{i}=\xi_{i}$ when $i$ is one of the end indices (i.e., $i=i_{-}-L_{-} \beta-L_{-}-1$ or $i=i_{-}-L_{-} \beta+L_{-}$), with $\eta_{i} \in J_{i}$, and with

$$
\sum H\left(\eta_{i}, \eta_{i+1}\right)<\sum H\left(\xi_{i}, \xi_{i+1}\right),
$$

where both sums are taken over the range of indices $i_{-}-L_{-} \beta-L_{-}-1 \leq$ $i \leq i_{-}-L_{-} \beta+L_{-}-1$. The existence of such a sequence $\eta_{i}$ contradicts the minimality of the sequence $\xi_{i}$. This contradiction will show that $\xi_{i} \neq a+\beta$ when $i=i_{-}-L_{-} \beta-1$ or $i=i_{-}-L_{-} \beta$. 
The first step towards obtaining this inequality is to obtain a lower bound on $\sum H\left(\xi_{i}, \xi_{i+1}\right)$. To describe this lower bound, we need a lot of notation, which we introduce next.

We set $v_{-}=\xi_{i}$ for $i=i_{-}-L_{-} \beta+L_{-}$and $v_{+}=\xi_{i}$ for $i=i_{-}-L_{-} \beta-$ $L_{-}-1$ (so that $v_{-}<a+\beta-1<a+\beta+1<v_{+}$). We let $W$ be the set of $x \in[a+\beta-1, a+\beta]$ that satisfy $H(x, x)=0$. (By the definition of $H$, we have $\min _{x \in \mathbb{R}} H(x, x)=0$.) For each bounded component $J$ of $\mathbb{R} \backslash W$, we let $x^{J}=\left(\ldots, x_{i}^{J}, \ldots\right)$ be a minimal configuration (for $H$ ) of rotation symbol 0 - that lies entirely in the interval $J$. By Aubry's theory (discussed in $\S 2$ ), such a configuration exists and satisfies $x_{i}^{J} \rightarrow J_{+}$, as $i \rightarrow-\infty$ and $x_{i}^{J} \rightarrow J_{-}$, as $i \rightarrow+\infty$, where $J=\left(J_{-}, J_{+}\right)$. There may be more than one such configuration for a given $J$, but we select, for each $J$, exactly one such configuration $\left(\ldots, x_{i}^{J}, \ldots\right)$. We let $w_{-}$and $w_{+}$be the least and greatest elements of $W$, resp. Note that $v_{-}<a+\beta-1<w_{-}<a+\beta<w_{+}<$ $a+\beta+1<v_{+}$. We let $\left(x_{i}^{-}\right)_{i \leq 0}$ be a configuration satisfying (a) $x_{0}^{-}=v_{-}$; (b) $x_{i}^{-} \in[a+\beta-1, a+\beta]$, for $i \leq-1$; and (c) $x_{i}^{-} \rightarrow w_{-}$as $i \rightarrow-\infty$. We suppose furthermore that $\left(\ldots, x_{i}^{-}, \ldots, x_{0}^{-}\right)$is minimal (for $H$ ) subject to these three conditions. Likewise, we let $\left(x_{i}^{+}\right)_{i \geq 0}$ be a configuration satisfying (a) $x_{0}^{+}=v_{+}$; (b) $x_{i}^{+} \in[a+\beta, a+\beta+1]$, for $i \geq 1$; and (c) $x_{i}^{+} \rightarrow w_{+}$as $i \rightarrow+\infty$. We suppose furthermore that $\left(x_{0}^{+}, \ldots, x_{i}^{+}, \ldots\right)$ is minimal (for $H$ ) subject to these three conditions.

For each bounded component $J$ of $\mathbb{R} \backslash W$, we let $D_{J}=\sum_{i=-\infty}^{\infty} H\left(x_{i}^{J}, x_{i+1}^{J}\right)$. We let $D_{+}=\sum_{i=0}^{\infty} H\left(x_{i}^{+}, x_{i+1}^{+}\right)$and $D_{-}=\sum_{i=-\infty}^{-1} H\left(x_{i}^{-}, x_{i+1}^{-}\right)$. From equations (8.1) and (8.2), together with the fact that these configurations are minimal (for $H$ and the appropriate boundary conditions in the case of $x^{-}$and $x^{+}$), it follows that all these sums are absolutely convergent and that

$$
\begin{aligned}
& D_{J} \geq \int_{J} \partial_{2} H(y, y+) d y, \\
& D_{+} \geq \int_{w_{+}}^{v_{+}} \partial_{2} H(y, y+) d y, \\
& D_{-} \geq \int_{v_{-}}^{w_{-}} \partial_{2} H(y, y+) d y .
\end{aligned}
$$

Here, we use the fact that the first and third summands on the right side of (8.2) are nonnegative.

Let $D=D_{-}+\sum_{J} D_{J}+D_{+}$, where the sum is taken over all bounded components $J$ of the complement of $W$ in $\mathbb{R}$. This sum is also absolutely convergent, again by equation (8.2). Moreover, by the minimality properties of the configurations $x_{-}, x_{+}$, and $x_{J}$, and equations (8.1) and (8.2), we have

$$
D<\sum_{i=m}^{n-1} H\left(\zeta_{i}, \zeta_{i+1}\right)
$$


for any sequence $\zeta_{m}, \ldots, \zeta_{n}$ whose end terms are $\zeta_{m}=v_{+}$and $\zeta_{n}=v_{-}$, and which satisfies $\zeta_{i} \in[a+\beta-1, a+\beta+1]$, for $m<i<n$. Moreover, we have

$$
D+P_{0-}^{H}(a)<\sum_{i=m}^{n-1} H\left(\zeta_{i}, \zeta_{i+1}\right)
$$

if, in addition, $\zeta_{i}=a+\beta$, for some $i$ between $m$ and $n$. This follows from the minimality properties of the configurations $x_{-}, x_{+}$, and $x_{J}$, equations (8.1) and (8.2), and the fact that $v_{+}>a+\beta+1$ and $v_{-}<a+\beta-1$. In particular,

$$
D+P_{0-}^{H}(a)<\sum H\left(\xi_{i}, \xi_{i+1}\right)
$$

where the sum is taken over $i_{-}-L_{-} \beta-L_{-}-1 \leq i \leq i_{-}-L_{-} \beta+L_{-}-1$. This completes the first step.

We now begin the construction of the sequence $\eta_{i}$. For the end indices (i.e., $i=i_{-}-L_{-} \beta-L_{-}-1$ and $\left.i=i_{-}-L_{-} \beta+L_{-}\right)$, we set $\eta_{i}=\xi_{i}$. We will choose the $\eta_{i}$ to be a monotone nonincreasing sequence that is a subset of

$$
V=W \cup \bigcup_{J}\left\{x_{i}^{J}\right\}_{i \in \mathbb{Z}} \cup\left\{x_{i}^{-}\right\}_{i \leq 0} \cup\left\{x_{i}^{+}\right\}_{i \geq 0},
$$

where $J$ ranges over all bounded components of $\mathbb{R} \backslash W$. Then we have by equation (8.2)

$$
D \leq \sum H\left(\eta_{i}, \eta_{i+1}\right)<D+\Delta D
$$

where

$$
\Delta D=\sum_{E} \mu_{H}\left(\Delta_{i}\right)
$$

$\Delta_{i}$ is the triangle $\left\{(y, z): \eta_{i+1} \leq z \leq y \leq \eta_{i}\right\}$, and the sum is taken over the set $E$ of $i$ satisfying $i_{-}-L_{-} \beta-L_{-}-1 \leq i<i_{-}-L_{-} \beta+L_{-}$such that the open interval $\left(\eta_{i+1}, \eta_{i}\right)$ contains an element of $V$.

Thus, our problem is to choose the sequence $\eta_{i}$ so as to make $\Delta D<P_{0-}^{H}(a)$. For this, we use the inequality (3.2) of [17]:

$$
\mu_{H}\left([x, \xi]^{2}\right) \leq(\xi-x) \nu_{H}^{2}(x, \xi)
$$

for real numbers $x<\xi$, where $\nu_{H}^{2}$ is the unique Borel measure on $\mathbb{R}$ such that

$$
\nu_{H}^{2}(y, z]=\theta(z-y)+\partial_{2} h(y, y+)-\partial_{2} h(z, z+)
$$


(We could just as well use the corresponding inequality involving $\nu_{H}^{1}$.) We will also use the fact that $\nu_{H}^{2}(x, x+1]=\theta$, for any $x \in \mathbb{R}$.

Both $V \cap[a+\beta-1, a+\beta]$ and $V \cap[a+\beta, a+\beta+1]$ are closed subsets of these intervals, and neither contains $a+\beta$, since $P_{0+}^{H}(a+\beta)=P_{0+}^{H}(a)>0$. We construct subsets $V_{-}$and $V_{+}$of these sets, as follows. We let $v_{1}$ be the least point of $V \cap[a+\beta-1, a+\beta]$. For $i>1$, we define $v_{i}$, as follows. If there exists $x>v_{i-1}$ in $V \cap[a+\beta-1, a+\beta]$ such that $\mu_{H}\left(\left[v_{i-1}, x\right]^{2}\right) \leq \varepsilon_{-}^{2} \theta$, we let $v_{i}$ be the greatest such $x$. (The quantity $\varepsilon_{-}$was defined just before the statement of Lemma b.) If no such $x$ exists, there are two possibilities: either there exists $x \in V \cap[a+\beta-1, a+\beta]$ with $x>v_{i-1}$ and the least such $x$ satisfies $\mu_{H}\left(\left[v_{i-1}, x\right]^{2}\right)>\varepsilon_{-}^{2} \theta$ or $v_{i-1}$ is the greatest element of $V \cap[a+\beta-1, a+\beta]$. In the former case, we let $v_{i}$ be the least $x \in V \cap[a+\beta-1, a+\beta]$ with $x>v_{i-1}$. In the latter case we terminate the process and define $V_{-}$to be the set $\left\{v_{1}, \ldots, v_{i-1}\right\}$. We define a subset $V_{+}$of $[a+\beta, a+\beta+1]$ in a similar way.

Let $e$ be the number of elements in $V_{-}$, so $V_{-}=\left\{v_{1}, \ldots, v_{e}\right\}$. By (8.3) and the construction of the sequence $v_{1}, \ldots, v_{e}$, we have

$$
\left(v_{2 i+1}-v_{2 i-1}\right)\left(\nu_{H}^{2}\left(v_{2 i-1}, v_{2 i+1}\right] / \theta\right) \geq \mu_{H}\left(\left[v_{2 i-1}, v_{2 i+1}\right]^{2}\right) / \theta>\varepsilon_{-}^{2} .
$$

Therefore,

$$
\left(v_{2 i+1}-v_{2 i-1}+\nu_{H}^{2}\left(v_{2 i-1}, v_{2 i+1}\right] / \theta\right)^{2} \geq 4\left(v_{2 i+1}-v_{2 i}\right)\left(\nu_{H}^{2}\left(v_{2 i-1}, v_{2 i+1}\right] / \theta\right)>4 \varepsilon_{-}^{2} \text {, }
$$

so

$$
\left(v_{2 i+1}-v_{2 i-1}\right)+\nu_{H}^{2}\left(v_{2 i-1}, v_{2 i+1}\right] / \theta>2 \varepsilon_{-} .
$$

Since $\nu_{H}^{2}(x, x+1]=\theta$, for every $x \in \mathbb{R}$, and $v_{1}<\cdots<v_{e}<v_{1}+1$, it follows from this last inequality that the number $e$ of elements in $V_{-}$is $\leq 2\left[\varepsilon_{-}^{-1}\right]+1=L_{-}^{0}<L_{-}$. Similarly, $V_{+}$has at most $L_{-}$elements.

Let $x_{-}$be an element of $[a+\beta-1, a+\beta]$ such that $H\left(x_{-}, x_{-}\right)=0$. Let $x_{+}$be an element of $[a+\beta, a+\beta+1]$ such that $H\left(x_{+}, x_{+}\right)=0$. We have that $x_{-}$(resp. $x_{+}$) is between the least and the greatest element of $V_{-}$(resp. $\left.V_{+}\right)$, since these are the least and greatest members of $V \cap[a+\beta-1, a+\beta]$ (resp. $V \cap[a+\beta, a+\beta+1]$ ) and $x_{-}$(resp. $\left.x_{+}\right)$is a member of this set.

Now we can finish constructing the sequence $\eta_{i}$. For $i_{-}-L_{-} \beta-L_{-} \leq i<$ $i_{-}-L_{-} \beta$, we let the $\eta_{i}$ be the members of $V_{+}$, each occurring just once. In addition, we let $x_{+}$occur in this sequence as many times as is required for the number of elements in the sequence to be $L_{-}$. Similarly, for $i_{-}-L_{-} \beta \leq i<$ $i_{-}-L_{-} \beta+L_{-}$, we let the $\eta_{i}$ be the members of $V_{-}$each occurring just once. In addition, we let $x_{-}$occur in this sequence as many times as is required for the number of elements in the sequence to be $L_{-}$. These elements should appear in monotone nonincresing order.

We have $\Delta D=\sum_{E} \mu_{H}\left(\Delta_{i}\right)<2\left(2\left[\varepsilon_{-}^{-1}\right]+1\right) \varepsilon_{-}^{2} \theta \leq P_{0-}^{H}(a) / 4$. The last inequality is a consequence of the definition of $\varepsilon_{-}$. The first inequality is a consequence of the fact that $E$ has at most as many elements as $V_{-} \cup V_{+}$, which has 
$2\left(2\left[\varepsilon_{-}^{-1}\right]+1\right)$, and the fact that $i \in E$ implies that $\mu_{H}\left(\left[\eta_{i+1}, \eta_{i}\right]\right) \leq \varepsilon_{-}^{2} \theta$. For, if $\mu_{H}\left(\left[\eta_{i+1}, \eta_{i}\right]\right)>\varepsilon_{-}^{2} \theta$, then the open interval $\left(\eta_{i+1}, \eta_{i}\right)$ contains no element of $V$, by the definitions of $V_{-}$and $V_{+}$.

Thus, $\sum H\left(\eta_{i}, \eta_{i+1}\right)<\sum H\left(\xi_{i}, \xi_{i+1}\right)$ and we have the desired contradiction.

We set $L_{+}^{0}=2\left[\varepsilon_{+}^{-1}\right]+1$, where $\varepsilon_{+}$is the unique positive solution of the equation $2 \varepsilon_{+}+\varepsilon_{+}^{2}=P_{0+}^{H}(b) / 8 \theta$.

Lemma c. If $L_{+} \geq L_{+}^{0}$ then $\xi_{i} \neq b+\beta$ when $i=i_{+}+L_{+} \beta-1$ or $i=i_{+}+L_{+} \beta$ and $1 \leq \beta \leq l_{+}-1$.

Proof. Same as the proof of Lemma b.

Lemma d. The function $H(x, x)$ takes its minimum value in the interval $\left[\xi_{\hat{i}}, \min (a, b)\right]$ at $x=\xi_{\hat{i}}$.

Proof. Suppose that $H(x, x) \leq H\left(\xi_{\hat{i}}, \xi_{\hat{i}}\right)$ for some $\xi_{\hat{i}}<x \leq \min (a, b)$. For $i_{-} \leq i<i_{+}$, let $\eta_{i}=\xi_{i}$ if $\xi_{i} \geq x$ and $\eta_{i}=x$ if $\xi_{i} \leq x$. Lemma a and equation (8.2) imply

$$
\sum H\left(\eta_{i}, \eta_{i+1}\right)<\sum H\left(\xi_{i}, \xi_{i+1}\right),
$$

where both sums are taken over the range of indices $i_{-} \leq i \leq i_{+}-2$. But this contradicts the assumption that $\xi$ is $\mathscr{J}$-minimal.

We set

$$
\delta=\left(\min \left\{H(x, x): x \in I_{a b}\right\} / 2|b-a| \nu_{H}^{2}\left(I_{a b}\right)\right)^{1 / 2},
$$

where $I_{a b}$ is the closed interval with endpoints $a$ and $b$. In the case that $a<b$, we set $K_{-}=1$ and $K_{+}=\left[2 \delta^{-1}+1\right]$. In the case that $b<a$, we set $K_{+}=1$ and $K_{-}=\left[2 \delta^{-1}+1\right]$.

Lemma e. If $H\left(\xi_{\hat{i}}, \xi_{\hat{i}}\right) \leq \min \left\{H(x, x): x \in I_{a b}\right\} / 2$, and $i_{-}<j<i_{+}, j-i_{-} \geq$ $K_{-}$, and $i_{+}-j>K_{+}$, then $\xi_{j}<\min (a, b)$. In particular, $\max \left(\xi_{i(0)-1}, \xi_{i(0)}\right)<$ $\min (a, b)$.

Proof. The last statement follows from the first, in view of the assumption in Theorem 8.1 that $i_{0}-i_{-}>K_{-}$and $i_{+}-i_{0}>K_{+}$.

Now we prove the first. By assumption, $\xi_{i} \leq a$ for $i_{-} \leq i<i_{0}$ and $\xi_{i} \leq b$ for $i_{0} \leq i<i_{+}$. By Lemma a, $\xi_{i}$ is weakly monotone decreasing for $i$ running from $i_{-}$to $\hat{i}$ and weakly monotone increasing for $i$ running from $\hat{i}$ to $i_{+}$. Moreover, $\xi_{\hat{i}}<\min (a, b)$ by the assumption that $H\left(\xi_{\hat{i}}, \xi_{\hat{i}}\right) \leq$ $\min \left\{H(x, x): x \in I_{a b}\right\} / 2$. Note that $\min \left\{H(x, x): x \in I_{a b}\right\}>0$ since $a, b$ are both in the same complementary interval $\left(c, c^{\prime}\right)$ of $\pi\left(M_{F, 0}\right)$ and $\pi\left(M_{F, 0}\right)=$ $\{x: H(x, x)=0\}$, in view of equation (8.1).

If $i_{-}<\widehat{i}$, then $\xi_{i_{-}+1}<\xi_{i_{-}}$. For, otherwise, by Lemma a, we would have $\xi_{i_{-}+1}=\xi_{i_{-}}$, and it would be possible, in view of equation (8.2) and Lemma d, to 
decrease $\sum_{i=i_{-}}^{\hat{i}-1} H\left(\xi_{i}, \xi_{i+1}\right)$ by deleting $\xi_{i_{-}+1}$ from the sequence $\xi_{i_{-}}, \ldots, \xi_{\hat{i}}$, and adjoining a second instance of $\xi_{\hat{i}}$ at the end of this sequence. Similarly, if $\hat{i}<i_{+}-1$, then $\xi_{i_{+}-2}<\xi_{i_{+}-1}$.

Consider the case $a<b$. Since $j-i_{-} \geq K_{-}=1$, we have $j>i_{-}$. Thus, if $\hat{i} \geq j$, we have $\xi_{j} \leq \xi_{i_{-}+1}<\xi_{i_{-}} \leq a$. Thus, to prove the lemma in the case that $a<b$, it is enough to consider the subcase when $j>\hat{i}$ and prove that $\xi_{j}<a$.

Consider an index $i$ that satisfies $j \leq i<i_{+}-2$ such that $a \leq \xi_{i}$. By equation (8.2),

$$
H\left(\xi_{i}, \xi_{i+1}\right)+H\left(\xi_{i+1}, \xi_{i+2}\right)-H\left(\xi_{i}, \xi_{i+2}\right) \geq H\left(\xi_{i+1}, \xi_{i+1}\right)-\mu_{H}(\Delta),
$$

where $\Delta=\left\{(y, z): \xi_{i} \leq y \leq z \leq \xi_{i+2}\right\}$. By the inequality (8.3), we have

$$
\mu_{H}(\Delta) \leq\left(\xi_{i+2}-\xi_{i}\right) \nu_{H}^{2}\left(\xi_{i}, \xi_{i+2}\right) \text {. }
$$

Now consider the effect of deleting $\xi_{i+1}$ from the sequence $\xi_{\hat{i}}, \ldots, \xi_{i_{+}-1}$ and adjoining a second instance of $\xi_{\hat{i}}$ at the beginning of the sequence. In view of (8.2), the inequality $H\left(\xi_{\hat{i}}, \xi_{\hat{i}}\right) \leq \min \left\{H(x, x): x \in I_{a b}\right\} / 2$ and the last two inequalities above, this operation will decrease the sum $\sum_{i=\hat{i}}^{i_{+}-2} H\left(\xi_{i}, \xi_{i+1}\right)$, provided that $\left(\xi_{i+2}-\xi_{i}\right) \nu_{H}^{2}\left(\xi_{i}, \xi_{i+2}\right)<\min \left\{H(x, x): x \in I_{a b}\right\} / 2$. From the minimality of $\xi$, we may therefore conclude that

$$
\left(\xi_{i+2}-\xi_{i}\right) \nu_{H}^{2}\left(\xi_{i}, \xi_{i+2}\right) \geq \min \left\{H(x, x): x \in I_{a b}\right\} / 2,
$$

when $i_{0} \leq i<i_{+}-2$ and $a \leq \xi_{i}$. Therefore

$$
\left(\frac{\xi_{i+2}-\xi_{i}}{b-a}+\frac{\nu_{H}^{2}\left(\xi_{i}, \xi_{i+2}\right)}{\nu_{H}^{2}(a, b)}\right)^{2} \geq \frac{2 \min \left\{H(x, x): x \in I_{a b}\right\}}{(b-a) \nu_{H}^{2}(a, b)} .
$$

Therefore, we have

$$
\sum\left(\frac{\xi_{i+2}-\xi_{i}}{b-a}+\frac{\nu_{H}^{2}\left(\xi_{i}, \xi_{i+1}\right)}{\nu_{H}^{2}(a, b)}\right) \geq 2 e \delta
$$

where $e$ is the number of terms in the sum. Therefore, if $e>\delta^{-1}$, we have

$$
\sum \xi_{i+2}-\xi_{i}>b-a \text { or } \sum \nu_{H}^{2}\left(\xi_{i}, \xi_{i+2}\right)>\nu_{H}^{2}(a, b) \text {. }
$$

Taking into account the fact that $\xi_{j} \leq \cdots \leq \xi_{i_{+}-1} \leq b$, we see that $\xi_{j}<a$ if $i_{+}-1-j>2 \delta^{-1}$.

This is the required result when $a<b$. The case $b<a$ may be treated similarly.

Lemma f. $\left(i_{+}-i_{-}\right) H\left(\xi_{\hat{i}}, \xi_{\hat{i}}\right) \leq 2 \theta$.

Proof. Let $\eta_{i}=\xi_{i}$ for $i=i_{-}-1$ and $i=i_{+}$and let $\eta_{i}=c$ for $i_{-} \leq i \leq i_{+}-1$.

Then

$$
0 \leq \sum_{i_{-}}^{i_{+}}\left(H\left(\eta_{i-1}, \eta_{i}\right)-H\left(\xi_{i-1}, \xi_{i}\right)\right) \leq 2 \theta-\left(i_{+}-i_{-}\right) H\left(\xi_{\hat{i}}, \xi_{\hat{i}}\right) .
$$


The first inequality is a consequence of the assumption that the sequence $\xi_{i}$ is $\mathscr{J}$-minimal. The second inequality follows from equation (8.2), Lemma $\mathrm{d}$, and inequality (8.3), together with the fact that $\nu_{H}^{2}(x, x+1]=\theta$, for any $x \in \mathbb{R}$.

Lemma g. For every $\varepsilon>0$, there exists a positive integer $K$ such that if $i_{+}-i_{-}>$ $K+K_{+}+K_{-}$, then $\mu_{H}\left(\left(c, \xi_{\hat{i}}\right)^{2}\right)<\varepsilon$.

Proof. Because $\mu_{H}(c, y)^{2} \rightarrow 0$ as $y \downarrow c$, it is enough to prove that for every $\delta>0$ there exists a positive integer $K$ such that if $i_{+}-i_{-}>K+K_{+}+K_{-}$, then $\xi_{\hat{i}}-c<\delta$. Moreover, since $H(c, c)=0, H(y, y)>0$, for $c<y<c^{\prime}$, and $H$ is continuous, it is enough to prove that for every $\eta>0$ there exists a positive integer $K$ such that if $i_{+}-i_{-}>K+K_{+}+K_{-}$, then $H\left(\xi_{\hat{i}}, \xi_{\hat{i}}\right)<\eta$. This follows from Lemma $f$.

Note that $K$ depends on $H, c, a$, and $b$. This is the case also in the next two lemmas.

Lemma $\mathbf{h}$. There exists a positive integer $K$ such that if $i_{+}-i_{-}>K+K_{+}+K_{-}$, then $\xi_{j} \neq a$ if $j=i_{-}$or $j=i_{-}-1$.

Proof. First, we consider the case $j=i_{-}-1$. By Aubry's theory, there exists a minimal configuration $\left(\ldots, \eta_{i}, \ldots\right)$ of rotation symbol $0-$ such that $\eta_{i} \rightarrow c^{\prime}$ as $i \rightarrow-\infty$ and $\eta_{i} \rightarrow c$ as $i \rightarrow+\infty$. We may choose the indexing so that $\eta_{i_{-}}<a<\eta_{i_{-}-1}$.

Then $\eta_{j}<\xi_{j}$ if $j=i_{-}-L_{-}-1$ or $j=i_{+}$, by the assumption that $\xi$ is a $\mathscr{J}$-configuration. Consequently, $\eta_{j}<\xi_{j}$ for $i_{-}-L_{-}-1 \leq j \leq i_{+}$, by the usual modification of the Aubry crossing lemma to the case when one of the configurations is minimal and the other is $\mathscr{J}$-minimal. In particular, $a<\eta_{i_{-}-1}<\xi_{i_{-}-1}$.

Now we consider the more difficult case, when $j=i_{-}$. We will suppose that $\xi_{i_{-}}=a$ and obtain a contradiction by showing that there exists a sequence $\eta_{i}$ with $i$ running from $i_{-}-L_{-}-1$ to $i_{+}+L_{+}$, with $\eta_{i}=\xi_{i}$ when $i$ is one of the end indices (i.e., $i=i_{-}-L_{-}-1$ or $i=i_{+}+L_{+}$), with $\eta_{i} \in J_{i}$, and with

$$
\sum H\left(\eta_{i}, \eta_{i+1}\right)<\sum H\left(\xi_{i}, \xi_{i+1}\right),
$$

where both sums are taken over the range of indices $i_{-}-L_{-}-1 \leq i<i_{+}+L_{+}$.

The first step toward the proof of this inequality is to obtain a lower bound for $\sum H\left(\xi_{i}, \xi_{i+1}\right)$, which requires the following notation.

We set $v_{-}=\xi_{i}$ for $i=i_{-}-L_{-}-1$ and $v_{+}=\xi_{i}$ for $i=i_{+}+L_{+}$. We let $A$ be the set of $x \in \mathbb{R}$ that satisfies the equation $H(x, x)=0$. For each bounded component $J$ of $\mathbb{R} \backslash A$, we let $x_{-}^{J}=\left(\ldots, x_{i-}^{J}, \ldots\right)$ and $x_{+}^{J}=\left(\ldots, x_{i+}^{J}, \ldots\right)$ be minimal configurations (for $H$ ) of rotation symbols $0_{-}$and $0+$, resp., that lie entirely in the interval $J$. We let $w_{-}$(resp. $w_{+}$) be the greatest element of $[c, a+1] \cap A$ (resp. $[c, b+1] \cap A)$. Note that $w_{-}<a+1 \leq v_{-}$ 
and $w_{+}<b+1 \leq v_{+}$. We let $x^{-}=\left(x_{i}^{-}\right)_{i \geq 0}$ be a configuration satisfying (a) $x_{0}^{-}=v_{-}$, (b) $x_{i}^{-} \in[c, a+1]$ for $i \geq 1$, and (c) $x_{i}^{-} \rightarrow w_{-}$as $i \rightarrow+\infty$. We suppose, in addition, that $x^{-}$is minimal in the set of configurations satisfying these three properties. We let $x^{+}=\left(x_{i}^{+}\right)_{i \leq 0}$ be a configuration satisfying (a) $x_{0}^{+}=v_{+},(\mathrm{b}), x_{i}^{+} \in[c, b+1]$ for $i \geq 1$, and (c) $x_{i}^{+} \rightarrow w_{+}$as $i \rightarrow-\infty$. We suppose, in addition, that $x^{+}$is minimal in the set of configurations satisfying these three properties. let

For each component $J$ of $\mathbb{R} \backslash A$, we let $D_{J \pm}=\sum_{i=-\infty}^{\infty} H\left(x_{i \pm}^{J}, x_{i+1 \pm}^{J}\right)$. We

$$
D_{-}=\sum_{i=0}^{\infty} H\left(x_{i}^{-}, x_{i+1}^{-}\right)+\sum_{J} D_{J-},
$$

where $J$ ranges over all components of $\mathbb{R} \backslash A$ contained in $[c, a+1]$. We let

$$
D_{+}=\sum_{i=-\infty}^{-1} H\left(x_{i}^{+}, x_{i+1}^{+}\right)+\sum_{J} D_{J+},
$$

where $J$ ranges over all components of $\mathbb{R} \backslash A$ contained in $[c, b+1]$. For the same reason as in the proof of Lemma $b$, it follows from equations (8.1), (8.2), and the minimality of $x_{ \pm}^{J}$ and $x^{ \pm}$that these sums are all absolutely convergent.

Let $D=D_{+}+D_{-}$. From (8.2), it follows that

$$
\sum_{i_{-}-L_{-}}^{i_{+}+L_{+}} H\left(\xi_{i-1}, \xi_{i}\right)>D-\mu_{H}\left(\left(c, \xi_{\hat{i}}\right)^{2}\right) .
$$

Moreover, our assumption that $\xi_{i_{-}}=a$ permits us to say more: we have

$$
\sum_{i_{-}-L_{-}}^{i_{+}+L_{+}} H\left(\xi_{i-1}, \xi_{i}\right)>D+P_{0-}^{H}(a)-\mu_{H}\left(\left(c, \xi_{\hat{i}}\right)^{2}\right) \text {. }
$$

This completes the first step: it is the required lower bound for $\sum H\left(\xi_{i}, \xi_{i+1}\right)$.

We now begin the construction of the sequence $\eta_{i}$. We choose $\eta_{i}$ for $i=$ $i_{-}-L_{-}-1, \ldots, i_{-}+L_{-}-1$ to be a monotone nonincreasing sequence that is a subset of

$$
V_{-}=(A \cap[c, a+1]) \cup\left\{x_{i}^{-}\right\}_{i \geq 0} \cup \bigcup_{J}\left\{x_{i-}^{J}\right\}_{i \in \mathbb{Z}},
$$

where $J$ runs over all components of $\mathbb{R} \backslash A$ in $[c, a+1]$. We choose the first member of this sequence to be $v_{-}$and the last member to be $c$. We choose $\eta_{i}$ for $i=i_{+}-L_{-}, \ldots, i_{+}+L_{+}$to be a monotone nondecreasing sequence that is a subset of

$$
V_{+}=(A \cap[c, b+1]) \cup\left\{x_{i}^{+}\right\}_{i \leq 0} \cup \bigcup_{J}\left\{x_{i+}^{J}\right\}_{i \in \mathbb{Z}},
$$

where $J$ runs over all components of $\mathbb{R} \backslash A$ in $[c, b+1]$. We choose the first member of this sequence to be $c$ and the last member to be $v_{+}$. We assume 
that $K$ is chosen large enough so that the hypothesis $i_{+}-i_{-}>K_{-}+K_{+}+K$ of Proposition 8.1 implies that $i_{-}+L_{-}-1 \leq i_{+}-L_{-}$. We set $\eta_{i}=c$ for $i_{-}+L_{-}-1 \leq i \leq i_{+}-L_{-}$.

We have

$$
D \leq \sum H\left(\eta_{i}, \eta_{i+1}\right)<D+\Delta D
$$

where

$$
\Delta D=\sum_{E} \mu_{H}\left(\Delta_{i}\right)
$$

and $\Delta_{i}$ is the triangle $\left\{(y, z): \eta_{i+1} \leq z \leq y \leq \eta_{i}\right\}$ when $\eta_{i+1}<\eta_{i}$ and the triangle $\left\{(y, z): \eta_{i} \leq y \leq z \leq \eta_{i+1}\right\}$. when $\eta_{i}<\eta_{i+1}$. The sum is taken over $E=E_{-} \cup E_{+}$, where $E_{-}$is the set of $i$ satisfying $i_{-}-L_{-}-1 \leq i<i_{-}+L_{-}-1$ such that the open interval $\left(\eta_{i+1}, \eta_{i}\right)$ contains an element of $V_{-}$and where $E_{+}$is the set of $i$ satisfying $i_{+}-L_{-} \leq i<i_{+}+L_{+}$such that the open interval $\left(\eta_{i}, \eta_{i+1}\right)$ contains an element of $V_{+}$.

Thus, our problem is to choose the sequence $\eta_{i}$ so as to make $\Delta D \leq P_{0-}^{H}(a)-$ $\mu_{H}\left(\left(c, \xi_{\hat{i}}\right)^{2}\right)$. Of course this should satisfy the further requirement $\eta_{i} \in J_{i}$.

We choose this sequence in the same way as we chose the corresponding sequence in the proof of Lemma b. (Note that the symbols $V, \eta_{i}$, etc. now have meanings different from those in the proof of Lemma b.) Thus, we choose a subset $V_{1}$ of $V_{-} \cap[a, a+1]$, a subset $V_{2}$ of $V_{-} \cap[c, a]$, a subset $V_{3}$ of $V_{+} \cap[c, b]$, and a subset $V_{4}$ of $V_{+} \cap[b, b+1]$, in the same way as we chose subsets $V_{-}$and $V_{+}$of $V \cap[a+\beta-1, a+\beta]$ and $V \cap[a+\beta, a+\beta+1]$ in the proof of Lemma b. The argument given in the proof of Lemma b shows that each of these subsets has $<L_{-}$elements. We let $x_{-}$(resp. $x_{+}$) be an element of $[a, a+1]$ (resp. $[b, b+1]$ ) such that $H\left(x_{-}, x_{-}\right)=0$ (resp. $\left.H\left(x_{+}, x_{+}\right)=0\right)$. For the same reason as in the proof of Lemma $b$, we have that $x_{-}$(resp. $x_{+}$) is between the least and the greatest element of $V_{1}$ (resp. $\left.V_{4}\right)$.

Now we can finish constructing the sequence $\eta_{i}$. For $i_{-}-L_{-} \leq i<i_{-}$, we let the $\eta_{i}$ be the members of $V_{1}$, each occurring just once. In addition, we let $x_{\text {_ }}$ occur in this sequence as many times as required for the number of elements in the sequence to be $L_{-}$. For $i_{-} \leq i<i_{-}+L_{-}$, we let the $\eta_{i}$ be the members of $V_{2}$, each occurring just once. In addition, we let $c$ occur at the end of this sequence as many times as required for the number of elements in the sequence to be $L_{-}$. For $i_{+}-L_{-} \leq i<i_{+}$, we let the $\eta_{i}$ be the members of $V_{3}$, each occurring just once. In addition, we let $c$ occur at the beginning of this sequence as many times as required for the number of elements in the sequence to be $L_{-}$. For $i_{+} \leq i<i_{+}+L_{+}$, we let the $\eta_{i}$ be the members of $V_{4}$, each occurring just once. In addition, we let $x_{+}$occur in this sequence as many times as required for the number of elements in the sequence to be $L_{-}$.

Just as in the proof of Lemma b, we have

$$
\Delta D \leq \sum_{E} \mu_{H}\left(\Delta_{i}\right)<4\left(2\left[\varepsilon_{-}^{-1}\right]+1\right) \varepsilon_{-}^{2} \theta<P_{0-}^{H}(a) / 2 .
$$


Thus, by Lemma g, $\sum H\left(\eta_{i}, \eta_{i+1}\right)<\sum H\left(\xi_{i}, \xi_{i+1}\right)$, provided that $K$ is chosen large enough so that $\mu_{H}\left(\left(c, \xi_{\hat{i}}\right)^{2}\right)<P_{0-}^{H}(a) / 2$.

Lemma i. There exists a positive integer $K$ such that if $i_{+}-i_{-}>K+K_{+}+K_{-}$, then $\xi_{j} \neq b$, if $j=i_{+}$or $i_{+}-1$.

Proof. Same as the proof of Lemma h.

Lemma j. $\xi_{i}<a+l_{-}$when $i=i_{-}-L_{-} l_{-}+1$ and $\xi_{i}<b+l_{+}$when $i=$ $i_{+}+L_{+} l_{+}-2$.

Proof. Consider the case $i=i_{-}-L_{-} l_{-}+1$. Then $\xi_{i-1} \in J_{i-1}=\left[a+l_{-}-1, a+\right.$ $\left.l_{-}\right]$, so $\xi_{i}<\xi_{i-1} \leq a+l_{-}$. Let $x \in\left(a+l_{-}-1, a+l_{-}\right)$be such that $H(x, x)=0$. If $\xi_{i-1} \leq x$, we have the required inequality. If $\xi_{i}=\xi_{i-1}>x$, then we may decrease $\sum H\left(\xi_{j}, \xi_{j+1}\right)$ by deleting $\xi_{i}$ from the sequence $\left(\ldots, \xi_{j}, \ldots\right)$ and adjoining $x$, with the new sequence indexed so that the monotonicity properties of Lemma a still hold. The fact that this operation decreases $H\left(\xi_{j}, \xi_{j+1}\right)$ follows immediately from equation (8.2). But this contradicts the assumption that $\xi$ is $\mathscr{J}$-minimal.

The other case may be treated in the same way.

Proof of Proposition 8.1. Immediately from Lemmas a, b, c, h, i, and j.

\section{More lemmas}

We consider the following relation between pairs of real numbers. We say $(\omega, a) \rightarrow\left(\omega^{\prime}, a^{\prime}\right)$ if there exist positive integers $K_{-1}, K, K_{1}$ such that the following holds: If $x^{-}=\left(\ldots, x_{i}^{-}, \ldots\right)$ and $x^{+}=\left(\ldots, x_{i}^{+}, \ldots\right)$ are minimal configurations with rotation numbers $\rho\left(x^{-}\right)=\omega$ and $\rho\left(x^{+}\right)=\omega^{\prime} ; \mathscr{J}^{ \pm}=$ $\left(\ldots, J_{i}^{ \pm}, \ldots\right)$ are defined by $J_{i}^{ \pm}=\left[a_{i}^{ \pm}, a_{i}^{ \pm}+1\right]$, where $a_{i}^{-}-a \in \mathbb{Z}, a_{i}^{+}-a^{\prime} \in$ $\mathbb{Z}$, and $x_{i}^{ \pm} \in\left[a_{i}^{ \pm}, a_{i}^{ \pm}+1\right)$; and $i_{-2}, i_{-1}, i_{1}, i_{2} \in\{-\infty\} \cup \mathbb{Z} \cup\{+\infty\}$ satisfy

$$
i_{-2}+K_{-1}<i_{-1}<i_{-1}+K<i_{1}<i_{1}+K_{1}<i_{2},
$$

then there exists an integer $l$ and a sequence $J_{i(-2)}^{*}, \ldots, J_{i(2)}^{*}$ of closed intervals such that

$$
J_{i}^{*}=J_{i}^{-}, \quad \text { for } i_{-2} \leq i \leq i_{-1}, \quad J_{i}^{*}=J_{i}^{+}+l, \quad \text { for } i_{1} \leq i \leq i_{2},
$$

and such that if $x=\left(x_{i(-2)}, \ldots, x_{i(2)}\right)$ is any segment of a configuration satisfying $x_{i} \in J_{i}^{*}$, for $i_{-2} \leq i \leq i_{2}$, and $x$ is $\mathcal{J}^{*}$-minimal, then $x_{i} \in \operatorname{int} J_{i}^{*}$, for $i_{-2}+K_{-1} \leq i \leq i_{2}-K_{1}$.

Clearly, this relation is transitive, i.e., $(\omega, a) \rightarrow\left(\omega^{\prime}, a^{\prime}\right)$ and $\left(\omega^{\prime}, a^{\prime}\right) \rightarrow$ $\left(\omega^{\prime \prime}, a^{\prime \prime}\right)$ imply $(\omega, a) \rightarrow\left(\omega^{\prime \prime}, a^{\prime \prime}\right)$.

Lemma 9.1. Consider real numbers $\omega, \omega^{\prime}, a$. Let $\Omega$ be the closed interval with endpoints $\omega$ and $\omega^{\prime}$. Suppose that there exists $P>0$ such that $P_{\lambda}(a)>P$ for all $\lambda \in \Omega$. Suppose that if $p / q \in \Omega$ is a rational number expressed in lowest terms, then $q>2 \theta / P$. Then $(\omega, a) \rightarrow\left(\omega^{\prime}, a\right)$. 
Remark. The hypothesis is symmetric in $\omega$ and $\omega^{\prime}$, so we also have $\left(\omega^{\prime}, a\right) \rightarrow$ $(\omega, a)$ by this lemma.

Proof. Let $x^{-}$and $x^{+}$be minimal configurations of rotation numbers $\omega$ and $\omega^{\prime}$, resp. Let $\mathcal{J}^{ \pm}=\left(\ldots, J_{i}^{ \pm}, \ldots\right)$ be defined in terms of $x^{ \pm}$and $a$ as above. Thus, $J_{i}^{ \pm}=\left[a_{i}^{ \pm}, a_{i}^{ \pm}+1\right]$, where $n_{i}^{ \pm}=a_{i}^{ \pm}-a_{i}$ is an integer, and $x_{i}^{ \pm} \in\left(a_{i}^{ \pm}, a_{i}^{ \pm}+1\right)$.

Let $\varepsilon=P / 2 \theta$. The first step in the proof is to show that there exists a positive integer $K$, independent of $x^{-}$and $x^{+}$, an integer $l$, and, for $i_{-1}+K<i_{1}$, an $\varepsilon$-restrained sequence $\left(\ldots, n_{i}, \ldots\right)$ such that $n_{i}=n_{i}^{-}$for $i \leq i_{-1}$ and $n_{i}=n_{i}^{+}+l$ for $i \geq i_{1}$.

The discussion preceding Proposition 7.4 shows how to do this. Indeed, the hypothesis that $q>2 \theta / P$ if $p / q$ is a rational number in $\Omega$ means that $\Omega$ is contained in a Farey interval of height $\varepsilon^{-1}$. The estimate $K(\lambda, \varepsilon) \leq 2 / \Delta_{\varepsilon}(\lambda)$, proved in $\S 7$, then implies that there exists $L \geq 0$ such that $K(\lambda, \varepsilon) \leq L$ for all $\lambda \in \Omega$. It follows from Lemma 7.3 that $n=\left(\ldots, n_{i}, \ldots\right)$ is $\varepsilon$-restrained if $n_{i}=\left[\lambda_{i}\right], \lambda_{i+1}-\lambda_{i}=r_{i}$, and the sequence $\left(\ldots, r_{i}, \ldots\right)$ has the property that for each $i \in \mathbb{Z}$, all of $r_{i-L}, \ldots, r_{i+L}$ are contained in a common Farey interval of height $2 L$. We have given examples preceding Proposition 7.4 of such sequences. The method of construction of these $\varepsilon$-sequences is easily seen to provide, for $i_{-1}+K<i_{1}$, an $\varepsilon$-restrained sequence $\left(\ldots, n_{i}, \ldots\right)$ such that $n_{i}=n_{i}^{-}$for $i \leq i_{-1}$ and $n_{i}=n_{i}^{+}+l$ for $i \geq i_{1}$.

Let $J_{i}^{*}=\left[a_{i}^{*}, a_{i}^{*}+1\right]$, where $a_{i}^{*}=n_{i}+a$, for $i_{-2} \leq i \leq i_{2}$, and $i_{-2}, i_{2}$ are integers satisfying $i_{-2} \leq i_{-1}$ and $i_{1} \leq i_{2}$. From Proposition 6.1, it follows that there are positive integers $K_{-1}, K_{1}$ such that if $i_{-2}+K_{-1} \leq i_{-1}$ and $i_{1}+K_{1} \leq i_{2}$, then for any constraint $\mathscr{J}=\left(\ldots, J_{i}, \ldots\right)$ satisfying $J_{i}=J_{i}^{*}$, for $i_{-2} \leq i \leq i_{2}$ and any $\mathscr{J}$-minimal configuration $x$, we have $x_{i} \in \operatorname{int} J_{i}$, $i_{-2}+K_{-1} \leq i \leq i_{2}-K_{1}$. In fact, the proof of Proposition 6.1 shows that in order to have this conclusion, it is enough to assume that $x=\left(x_{i(-2)}, \ldots, x_{i(2)}\right)$ is $\mathscr{J}^{*}$-minimal, where $\mathscr{J}^{*}$ is the segment $\left(J_{i(-2)}^{*}, \ldots, J_{i(-2)}^{*}\right)$. This is the required result.

In the proof of Lemma 9.3, we will need the following slight strengthening of Lemma 9.1.

Addendum. The various choices may be made in such a way that (in the notation of the proof above), if $\omega<\omega^{\prime}$, then $\left(\ldots, n_{i}-n_{i}^{-}, \ldots\right)$ and $\left(\ldots, n_{i}^{+}-n_{i}, \ldots\right)$ are nondecreasing sequences. If $\omega^{\prime}<\omega$ these are nonincreasing sequences.

Proof. There is no difficulty seeing that the construction above the $\varepsilon$-restrained sequence $\left(\ldots, n_{i}, \ldots\right)$ can be done in such a way that this additional property holds.

Lemma 9.2. If $\omega$ is irrational, $a, b \in \mathbb{R}$, and $P_{\omega}(a)$ and $P_{\omega}(b)$ are positive, then $(\omega, a) \rightarrow(\omega, b)$.

Proof. This follows from the proof of Proposition 6.2.

Lemma 9.3. Suppose $p / q$ is a rational number, $a, b$ both lie in the same complementary interval of $\pi\left(M_{f, p / q}\right)$ and satisfy $P_{p / q-}(a)>0$ and $P_{p / q+}(b)>0$. 
Then there exists $\varepsilon>0$ such that if $p / q-\varepsilon<\omega_{-}<p / q<\omega_{+}<p / q+\varepsilon$, then $\left(\omega_{-}, a\right) \rightarrow\left(\omega_{+}, b\right)$ and $\left(\omega_{+}, b\right) \rightarrow\left(\omega_{-}, a\right)$.

Proof. We will prove $\left(\omega_{-}, a\right) \rightarrow\left(\omega_{+}, b\right)$, the other case being similar.

In [17, Theorem 2.2], we derived the following estimates: $\left|P_{p / q+}(\xi)-P_{\omega}(\xi)\right| \leq$ $C \theta|\omega q-p|$ and $\left|P_{p / q-}(\xi)-P_{\omega}(\xi)\right| \leq C \theta|\omega q-p|$, where $C$ is independent of everything. It follows from these estimates that there exist $\varepsilon, P>0$ such that if $(p / q)-\varepsilon<\omega_{-}^{\prime}<p / q<\omega_{+}^{\prime}<(p / q)+\varepsilon$ then $P_{\omega_{-}^{\prime}}(a)>P$ and $P_{\omega_{+}^{\prime}}(b)>P$. We suppose that $\varepsilon>0$ is chosen so small that if $(p / q)-\varepsilon<p^{\prime} / q^{\prime}<(p / q)+\varepsilon$ and $p^{\prime} / q^{\prime} \neq p / q$, then $q^{\prime}>2 \theta / P$. Then it follows from Lemma 9.1 that $\left(\omega_{-}^{\prime}, a\right) \rightarrow\left(\omega_{-}^{\prime \prime}, a\right)$ and $\left(\omega_{+}^{\prime}, b\right) \rightarrow\left(\omega_{+}^{\prime \prime}, b\right)$ if $(p / q)-\varepsilon<\omega_{-}^{\prime}, \omega_{-}^{\prime \prime}<p / q<$ $\omega_{+}^{\prime}, \omega_{+}^{\prime \prime}<p / q+\varepsilon$

Note that the hypotheses of Proposition 8.1 are satisfied, since $M_{f, p / q}=$ $M_{F, 0}$ and $P_{p / q_{-}}(a)=P_{p / q_{-}}^{h}(a)=P_{0-}^{H}(a)$ and $P_{p / q+}(b)=P_{p / q_{+}}^{h}(b)=P_{0+}^{H}(b)$. We will use the notations of Proposition 8.1 throughout this proof. Also, we let $\omega_{-}, \omega_{+}$be fixed numbers satisfying the hypotheses of Lemma 9.3. We will suppose that $L_{ \pm}$are integers that satisfy $\omega_{-}<p / q-1 / q L_{-}$and $\omega_{+}>$ $p / q+1 / q L_{+}$, in addition to the condition $L_{ \pm} \geq L_{ \pm}^{0}$ of Proposition 8.1. In this proof, the integers $l_{-}$and $l_{+}$of Proposition 8.1 will be taken to be sufficiently large. How large they must be taken will be explained during the course of the proof. We set

$$
\begin{aligned}
& \widehat{J_{i}^{-}}=[a+\beta-1, a+\beta], \quad \text { if } i_{-}-L_{-} \beta \leq i<i_{-}-L_{-} \beta+L_{-} \text {and } \beta \in \mathbb{Z}, \\
& \widehat{J}_{i}^{+}=[b+\beta-1, b+\beta], \quad \text { if } i_{+}+L_{+} \beta-L_{+} \leq i<i_{+}+L_{+} \beta \text { and } \beta \in \mathbb{Z} \text {. }
\end{aligned}
$$

We set $\widehat{J}^{ \pm}=\left(\ldots, \widehat{J}_{i}^{ \pm}, \ldots\right)$. We let $\xi^{ \pm}=\left(\ldots, \xi_{i}^{ \pm}, \ldots\right)$ be the largest $\widehat{J}^{ \pm}$. minimal configuration (for $H$ ). In fact, $\xi^{ \pm}$is a minimal configuration (for $H$ ) of rotation symbol $\pm 1 / L_{ \pm}$. We let $\xi^{* \pm}$ be the minimal configuration (for h) of rotation symbol $p / q \pm 1 / q L_{ \pm}$such that $\xi_{q i}^{* \pm}=\xi_{i}^{ \pm}+p i$. We let $J_{\alpha}^{*-}$ be the closed interval $\left[a_{\alpha}^{*-}, a_{\alpha}^{*-}+1\right]$ defined by the conditions $a_{\alpha}^{*-}-a \in \mathbb{Z}$ and $\xi_{\alpha}^{*-} \in\left(a_{\alpha}^{*-}, a_{\alpha}^{*-}+1\right)$. Likewise, we let $J_{\alpha}^{*+}$ be the closed interval $\left[a_{\alpha}^{*+}, a_{\alpha}^{*+}+1\right]$ defined by the conditions $a_{\alpha}^{*+}-b \in \mathbb{Z}$ and $\xi_{\alpha}^{*+} \in\left(a_{\alpha}^{*+}, a_{\alpha}^{*+}+1\right)$. We let $J_{i}$ be as in Proposition 8.1 and set

$$
\begin{array}{ll}
J_{\alpha}^{*}=J_{\alpha}^{*-}, & \text { for } q\left(i_{-}-L_{-} l_{-}-L_{-}\right) \leq \alpha<q i_{-}, \\
J_{\alpha}^{*}=J_{\alpha}^{*+}, & \text { for } q\left(i_{+}-1\right)<\alpha \leq q\left(i_{+}+L_{+} l_{+}+L_{+}-1\right), \\
J_{\alpha}^{*}=J_{i}+p i, & \text { for } \alpha=q i \text { and } i_{-} \leq i<i_{+}, \\
J_{\alpha}^{*}=\mathbb{R}, & \text { for all other } \alpha \text { between } q i_{-} \text {and } q\left(i_{+}-1\right) .
\end{array}
$$

Thus, for each $\alpha$ satisfying $q /\left(i_{-}-L_{-} l_{-}-L_{-}\right) \leq \alpha \leq q\left(i_{+}+L_{+} l_{+}+L_{+}-1\right)$, we have defined a closed, connected, nonempty set $J_{\alpha}^{*}$.

We chose $\varepsilon>0$ so that if $(p / q)-\varepsilon<\omega_{-}^{\prime}, \omega_{-}^{\prime \prime}<p / q<\omega_{+}^{\prime}, \omega_{+}^{\prime \prime}<(p / q)+\varepsilon$ then the hypotheses of Lemma 9.1 apply to the pairs $\left(\omega_{-}^{\prime}, a\right),\left(\omega_{-}^{\prime \prime}, a\right)$ and $\left(\omega_{+}^{\prime}, b\right),\left(\omega_{+}^{\prime \prime}, b\right)$. 
Consequently, we may conclude that $\left(\omega_{-}^{\prime}, a\right) \rightarrow\left(\omega_{-}^{\prime \prime}, a\right)$ and $\left(\omega_{+}^{\prime}, b\right) \rightarrow$ $\left(\omega_{+}^{\prime \prime}, b\right)$. We will apply this with $\omega_{-}^{\prime}=\omega_{-}, \omega_{-}^{\prime \prime}=p / q-1 / q L_{-}, \omega_{+}^{\prime}=p / q+$ $1 / q L_{+}$, and $\omega_{+}^{\prime \prime}=\omega_{+}$. Thus, it follows from Lemma 9.1 that, given minimal configurations $x^{-}$and $x^{+}$of rotation numbers $\omega^{-}$and $\omega^{+}$, we can extend the definition of $J_{\alpha}^{*}$ to a range $i_{-2} \leq \alpha \leq i_{2}$, where $i_{-2}<q\left(i_{-}-L_{-} l_{-}-L_{-}\right)$ and $i_{2}>q\left(i_{+}+L_{+} l_{+}+L_{+}-1\right)$, with the following properties: Let $J_{\alpha}^{-}$and $J_{\alpha}^{+}$ be defined by $J_{\alpha}^{-}=\left[a_{\alpha}^{-}, a_{\alpha}^{-}+1\right]$, where $a_{\alpha}^{-}-a \in \mathbb{Z}$ and $x_{\alpha}^{-} \in\left(a_{\alpha}^{-}, a_{\alpha}^{-}+1\right)$, and $J_{\alpha}^{+}=\left[a_{\alpha}^{+}, a_{\alpha}^{+}+1\right]$, where $a_{\alpha}^{+}-b \in \mathbb{Z}$ and $x_{\alpha}^{+} \in\left(a_{\alpha}^{+}, a_{\alpha}^{+}+1\right)$. We require that $J_{\alpha}^{*}=J_{\alpha}^{-}+\lambda^{-}$for $i_{-2} \leq \alpha \leq i_{-1}$ and $J_{\alpha}^{*}=J_{\alpha}^{+}+\lambda^{+}$for $i_{1} \leq \alpha \leq i_{2}$, where $\lambda^{-}$and $\lambda^{+}$are suitable integers. Here $i_{-2}+K_{-1}<i_{-1}<q\left(i_{-}-L_{-} l_{-}-L_{-}\right)$and $q\left(i_{+}+L_{+} l_{+}+L_{+}-1\right)<i_{1}<i_{2}-K_{1}$, and $i_{-2}, K_{-2}, i_{-1}$ are chosen so that we can apply Lemma 9.1 to $x^{-}$(of rotation number $\omega_{-}$) and $\xi^{*-}$ (of rotation number $\left.p / q-1 / q L_{-}\right)$, and $i_{1}, K_{2}, i_{2}$ are chosen so that we can apply Lemma 9.1 to $\xi^{*+}$ and $x^{+}$. In order to apply Lemma 9.1 , we must also choose $l_{-}$and $l_{+}$to be sufficiently large. By choosing the extension $\mathscr{J}^{*}=\left(J_{i(-2)}^{*}, \ldots, J_{i(2)}^{*}\right)$ according to Lemma 9.1, we have that if $x=\left(x_{i(-2)}, \ldots, x_{i(2)}\right)$ is a $\mathcal{J}^{*}$-minimal segment of a configuration, then $x_{\alpha} \in J_{\alpha}^{*}$ for $i_{-2}+K_{-1} \leq \alpha<q i_{-}-K_{-1}^{*}$ and for $q\left(i_{+}-1\right)+K_{1}^{*}<\alpha \leq i_{2}-K_{1}$. Here, $K_{-1}^{*}$ and $K_{1}^{*}$ are integers that are chosen suitably large for the application of Lemma 9.1. We also suppose that the addendum to Lemma 9.1 is satisfied by $\mathcal{J}^{*}$.

The next step is to show that $x_{\alpha} \in \operatorname{int} J_{\alpha}^{*}$, for all $\alpha$ satisfying $q i_{-}$ $K_{-1}^{*} \leq \alpha \leq q\left(i_{+}-1\right)+K_{1}^{*}$. For this, we use a slight variant of Proposition 8.1: We replace the conclusion of Proposition 8.1 with the conclusion that if $\left(\xi_{i_{-}-L_{-} l_{-}-1}, \ldots, \xi_{i_{+}+L_{+} l_{+}}\right)$is a segment of a $\mathscr{J}$-configuration and minimal (for fixed end terms) among such segments, then $\xi_{i} \in$ int $J_{i}$ for $i_{-}-L_{-} l_{-}+1 \leq i<$ $i_{+}+L_{+} l_{+}-1$. The proof of Proposition 8.1 shows that this conclusion holds.

We will apply this variant of Proposition 8.1 to the segment $\left(\xi_{i_{-}-L_{-} l_{-}-1}, \ldots\right.$, $\xi_{i_{+}+L_{+} l_{+}}$), where $\xi_{i}=x_{q i}-p i$. This is clearly the segment of a $\mathscr{J}^{-}$-configuration, in view of the fact that $x$ is a $\mathscr{J}^{*}$-configuration and the easily verified fact that

$$
J_{q i}^{*}=J_{i}+p i \text { for } i_{-}-L_{-} l_{-}-1 \leq i \leq i_{+}+L_{+} l_{+}-1 .
$$

To apply this variant of Proposition 8.1, we need to show that the segment $\left(\xi_{i_{-}-L_{-} l_{-}-1}, \ldots, \xi_{i_{+}+L_{+} l_{+}}\right)$is minimal (subject to fixed end terms) among segments of $\mathscr{J}$-configurations.

To prove this, we consider a segment $\left(\widehat{\xi}_{i_{-}-L_{-} l_{-}-L_{-}}, \ldots, \widehat{\xi}_{i_{+}+L_{+} l_{+}+L_{+}-1}\right)$ of a $\mathscr{J}$-configuration that satisfies $\widehat{\xi}_{i}=\xi_{i}$ for $i=i_{-}-L_{-} l_{-}-L_{-}$and $i=$ $i_{+}+L_{+} l_{+}+L_{+}-1$ and that is minimal (for $H$ ), subject to fixed end terms. The proof of Proposition 5.1 shows that such a configuration exists. We set $\hat{x}_{q i}=\widehat{\xi}_{i}+p i$ for $i_{-}-L_{-} l_{-}-L_{-} \leq i \leq i_{+}+L_{+} l_{+}-1$. For $i_{-}-L_{-} l_{-}-L_{-} \leq$ $i<i_{+}+L_{+} l_{+}$, we let $\left(\widehat{x}_{q i}, \ldots, \widehat{x}_{\alpha}, \ldots, \widehat{x}_{q(i+1)}\right)$ be a segment that is minimal 
$($ for $h)$. Let $\hat{x}$ denote the segment $\left.\left(\widehat{x}_{q\left(i_{-}-L_{-} l_{-} L_{-}\right.}\right), \ldots, \widehat{x}_{q\left(i_{+}+L_{+} l_{+}-1\right)}\right)$. We will prove below that $\hat{x}$ is a segment of a $\mathscr{J}^{*}$-configuration. This implies that $\left(\xi_{i_{-}-L_{-} l_{-}-L_{-}}, \ldots, \xi_{i_{+}+L_{+} l_{+}+L_{+}-1}\right)$ is minimal (for $H$, subject to fixed end terms) and therefore its restriction $\left(\xi_{i_{-}-L_{-} l_{-}-1}, \ldots, \xi_{i_{+}+L_{+} l_{+}}\right)$has the same minimality property. For, $\left(\xi_{i_{-}-L_{-} l_{-}-L_{-}}, \ldots, \xi_{i_{+}+L_{+} l_{+}+L_{+}-1}\right)$ is defined as the restriction of a $\mathcal{J}^{*}$-minimal (for $h$ ) configuration $x$. Then the fact that $\hat{x}$ is a segment of a $\mathcal{J}^{*}$-configuration implies that

$$
\sum H\left(\xi_{i}, \xi_{i+1}\right) \leq \sum h\left(x_{\alpha}, x_{\alpha+1}\right) \leq \sum h\left(\widehat{x}_{\alpha}, \widehat{x}_{\alpha+1}\right)=\sum H\left(\widehat{\xi}_{i}, \widehat{\xi}_{i+1}\right)
$$

and therefore, by the minimality of $\widehat{\xi}$, we have equality.

To prove that $\hat{x}$ is a segment of a $\mathscr{J}^{*}$-configuration, we define configurations $\eta^{ \pm}, \eta^{* \pm}$, as follows. We let $\eta^{ \pm}$(resp. $\eta^{* \pm}$ ) be the smallest minimal configuration (for $H$, resp. $h$ ) of rotation symbol $\pm 1 / L_{ \pm}$(resp. $p / q \pm 1 / q L_{ \pm}$) that is larger than $\xi^{ \pm}$(resp. $\left.\xi^{* \pm}\right)$. It is clear that $\eta_{q i}^{* \pm}=\eta_{i}^{ \pm}+p i$. We have

$$
\begin{array}{ll}
a+\beta-1<\xi_{i}^{-}<\alpha+\beta<\eta_{i}^{-}, & \text {if } i=i_{-}-L_{-} \beta \text { and } \beta \in \mathbb{Z}, \\
a+\beta-1<\xi_{i}^{-}<\eta_{i}^{-}<\alpha+\beta, & \text { all other } i \in \mathbb{Z}, \\
b+\beta-1<\xi_{i}^{+}<b+\beta<\eta_{i}^{+}, & \text {if } i=i_{+}+L_{+} \beta-1 \text { and } \beta \in \mathbb{Z}, \\
b+\beta-1<\xi_{i}^{+}<\eta_{i}^{+}<b+\beta, & \text { all other } i \in \mathbb{Z},
\end{array}
$$

and $\eta_{\alpha}^{* \pm} \in \operatorname{int} J_{\alpha}^{* \pm}$ for all $\alpha$ that are not of the form $q\left(i_{-}-L_{-} \beta\right)$, with $\beta \in \mathbb{Z}$ (resp. $q\left(i_{+}+L_{+} \beta-1\right.$ ), with $\beta \in \mathbb{Z}$ ), by the definitions: $\xi^{ \pm}$is the largest $\widehat{J}^{ \pm}$. minimal configuration (for $H$ ) and $\eta^{ \pm}$is the smallest minimal configuration (for $H$ ) of rotation symbol $\pm 1 / L_{ \pm}$that is greater than $\xi^{ \pm}$, etc.

We have $\xi_{\alpha}^{*-} \leq x_{\alpha}$ for all $\alpha$. This is because $\xi^{*-}$ is a $\mathscr{J}^{*-}$-minimal configuration, $x$ is a $\mathscr{J}^{*}$-minimal configuration, $J_{\alpha}^{*-} \leq J_{\alpha}^{*}$, for every $\alpha$ (in the sense that the corresponding endpoints satisfy this inequality), and $J_{\alpha}^{*-}$ is strictly to the left of $J_{\alpha}^{*}$ for $\alpha$ large or small enough. Thus, the inequality $\xi_{\alpha}^{*-} \leq$ $x_{\alpha}$ follows from the relative Aubry crossing lemma, applied to the minimal configuration $\xi^{*-}$ and the $\mathscr{J}^{*}$-minimal configuration $x$. Similarly, $\xi_{\alpha}^{*+} \leq x_{\alpha}$, for all $\alpha$.

As a particular case of $\xi_{\alpha}^{*+} \leq x_{\alpha}$ for all $\alpha$, we have $\xi_{i}^{ \pm} \leq \xi_{i}$, for all $i$. Since $\widehat{\xi}_{i}=\xi_{i}$ for $i=i_{-}-L_{-} l_{-}-L_{-}$and $i=i_{+}+L_{+} l_{+}+L_{+}-1$, we have $\xi_{i}^{ \pm} \leq \widehat{\xi}_{i}$ for these two values of $i$. Using the relative Aubry crossing lemma again, we have $\xi_{i}^{ \pm} \leq \widehat{\xi}_{i}$ for $i_{-}-L_{-} l_{-}-L_{-} \leq i \leq i_{+}+L_{+} l_{+}+L_{+}-1$. From this and the Aubry crossing lemma, it follows that

$$
\xi_{\alpha}^{*-} \leq \hat{x}_{\alpha}, \quad \text { for } q\left(i_{-}-L_{-} l_{-}-L_{-}\right) \leq \alpha \leq q\left(i_{+}+L_{+} l_{+}+L_{+}-1\right) .
$$

We also have $\widehat{\xi}_{i} \leq a+\beta<\eta_{i}^{-}$, if $i=i_{-}-L_{-} \beta, \beta \in \mathbb{Z}$, and $1 \leq \beta \leq l_{-}+1$. by an inequality we already obtained for $\eta_{i}^{-}$and our assumption that $\widehat{\xi}$ is 
a segment of a $\mathscr{J}$-configuration. Thus, by our assumption that $\widehat{\xi}$ is a $\mathscr{J}$ minimal segment and the relative Aubry crossing lemma, we have $\hat{\xi}_{i} \leq \eta_{i}^{-}$for $i_{-}-L_{-} l_{-}-L_{-} \leq i \leq i_{-}-L_{-}$. By the definition of $\hat{x}$ and the Aubry crossing lemma, it follows that

$$
\widehat{x}_{\alpha} \leq \eta_{\alpha}^{*-}, \quad \text { for } q\left(i_{-}-L_{-} l_{-}-L_{-}\right) \leq \alpha \leq q\left(i_{-}-L_{-}\right) .
$$

A similar argument shows that

$$
\xi_{\alpha}^{*+} \leq \widehat{x}_{\alpha} \leq \eta_{\alpha}^{*+}, \quad \text { for } q\left(i_{+}+L_{+}-1\right) \leq \alpha \leq q\left(i_{+}+L_{+} l_{+}+L_{+}-1\right) .
$$

Thus $\hat{x}$ is a segment of a $\mathscr{J}^{*}$-configuration: to show that $\hat{x}_{\alpha} \in J_{\alpha}^{*}$, we use the fact that $\widehat{\xi}$ is a segment of a $\mathscr{J}$-configuration (which implies that $\hat{x}_{\alpha} \in J_{\alpha}^{*}$ when $\alpha=q i$ ) and the inequalities above, which show that $\hat{x}_{\alpha} \in J_{\alpha}^{*}$ when $\alpha$ is not of the form $q i$ and in one of the above ranges. In the remaining cases, $J_{\alpha}^{*}=\mathbb{R}$, so there is nothing to prove.

To summarize, we have proved that $\hat{x}$ is a segment of a $\mathcal{J}^{*}$-configuration. As discussed above, it then follows that $\left(\xi_{i_{-}-L_{-} l_{-}-L_{-}}, \ldots, \xi_{i_{+}+L_{+} l_{+}+L_{+}+1}\right)$ is minimal (subject to fixed end terms) among segments of $\mathscr{J}$-configurations.

Thus, we may apply the variant of Proposition 8.1 to $\left(\xi_{i_{-}-L_{-} l_{-}-1}, \ldots\right.$, $\left.\xi_{i_{+}+L_{+} l_{+}}\right)$. From this variant, we obtain $x_{\alpha} \in \operatorname{int} J_{\alpha}^{*}$ for $\alpha=q i$, where $i_{-}$ $L_{-} l_{-}^{+} 1 \leq i \leq i_{+}+L_{+} l_{+}-2$. We also have

$$
\begin{array}{ll}
\xi_{\alpha}^{*-} \leq x_{\alpha} \leq \eta_{\alpha}^{*-}, & \text { for } q\left(i_{-}-L_{-} l_{-}-L_{-}\right) \leq \alpha \leq q\left(i_{+}+L_{+} l_{+}+L_{+}-1\right), \\
\xi_{\alpha}^{*+} \leq x_{\alpha} \leq \eta_{\alpha}^{*+}, & \text { for } q\left(i_{+}+L_{+}-1\right) \leq \alpha \leq q\left(i_{+}+L_{+} l_{+}+L_{+}-1\right) .
\end{array}
$$

These inequalities may be proved in the same way that the corresponding inequalities for $\hat{x}$ were proved. (All that we used was that $\widehat{\xi}$ was $\mathcal{J}$-minimal, a property that we have just finished proving is enjoyed by $\left(\xi_{i_{-}-L_{-} l_{-}-L_{-}}, \ldots\right.$, $\left.\xi_{i_{+}+L_{+} l_{+}+L_{+}+1}\right)$.)

For $\alpha$ not of the form $q i$, we have either $J_{\alpha}^{*}=\mathbb{R}$ or $x_{\alpha} \in \operatorname{int} J_{\alpha}^{*}$ by one of the inequalities above. To summarize, we have proved

$$
\begin{array}{ll}
x_{\alpha} \in \operatorname{int} J_{\alpha}^{*}, & \text { for } i_{-2}+K_{-1} \leq \alpha \leq q i_{-}-K_{-1}^{*} \\
& \text { and for } q\left(i_{+}-1\right)+K_{1}^{*} \leq \alpha \leq i_{2}-K_{1} .
\end{array}
$$

Thus, it is enough to choose $l_{-}, l_{+}$large enough in order to have

$$
x_{\alpha} \in \operatorname{int} J_{\alpha}^{*}, \quad \text { for } i_{-2}+K_{-1} \leq \alpha \leq i_{2}-K_{1} .
$$

How large these must be chosen depends only on $K_{-1}^{*}$ and $K_{1}^{*}$. Furthermore, we choose $K$ so large that when $i_{-1}+K<i_{1}$, it is possible to find $i_{-}$and $i_{+}$that satisfy the hypothesis of Proposition 8.1 (on the size of $i_{+}-i_{-}$) and also the conditions $i_{-1}<q\left(i_{-}-L_{-} l_{-}-L_{-}\right)$and $q\left(i_{+}+L_{+} l_{+}+L_{+}-1\right)<i_{1}$, which we needed in the first step of the proof. With these choices, we have the required result. 
Our main results concern orbits within a Birkhoff region of instability $R$. In $\S 4$, we denoted the lower and upper boundaries of $R$ by $\Gamma_{-}$and $\Gamma_{+}$. By putting together Lemmas 9.1-9.3, we obtain

Lemma 9.4. If $\rho\left(\Gamma_{-}\right)<\omega_{-}, \omega_{+}<\rho\left(\Gamma_{+}\right), \omega_{-}, \omega_{+}$are irrational numbers, and $a, b$ are real numbers with $P_{\omega_{-}}(a)>0, P_{\omega_{+}}(b)>0$, then $\left(\omega_{-}, a\right) \rightarrow\left(\omega_{+}, b\right)$.

Proof. Consider a rational number $p / q$ and a complementary interval $\left(c, c^{\prime}\right)$ of $\pi\left(M_{p / q}\right)$. Let $(c, \gamma),\left(c^{\prime}, \gamma^{\prime}\right) \in M_{p / q}$. If $P_{p / q+}$ (resp. $\left.P_{p / q-}\right)$ vanishes identically on $\left(c, c^{\prime}\right)$, then there exists a Lipschitz curve $\Gamma_{c, c^{\prime}}$ in $M_{p / q+}$ (resp. $\left.M_{p / q-}\right)$ connecting $(c, \gamma)$ and $\left(c^{\prime}, \gamma^{\prime}\right)$. Moreover, $\widetilde{\Gamma}_{c, c^{\prime}}=\Gamma_{c, c^{\prime}} \cup f\left(\Gamma_{c, c^{\prime}}\right) \cup$ $\cdots \cup f^{q-1}\left(\Gamma_{c, c^{\prime}}\right)$ is invariant under $f$.

If for each complementary interval $\left(c, c^{\prime}\right)$ of $\pi\left(M_{p / q}\right)$ at least one of $P_{p / q-}$ or $P_{p / q+}$ vanishes identically on $\left(c, c^{\prime}\right)$, then there is an invariant curve of rotation number $p / q$. It may be constructed as a union of curves $\widetilde{\Gamma}_{c, c^{\prime}}$.

Therefore, if $\rho\left(\Gamma_{-}\right)<p / q<\rho\left(\Gamma_{+}\right)$, then there exists at least one complementary interval $\left(c, c^{\prime}\right)$ of $\pi\left(M_{p / q}\right)$ on which neither $P_{p / q-}$ nor $P_{p / q+}$ vanishes identically. From Lemma 9.3 , it follows that there exists $\varepsilon>0$ such that if $p / q-\varepsilon<\omega_{-}<p / q<\omega_{+}<p / q+\varepsilon$, then $\left(\omega_{-}, a\right) \rightarrow\left(\omega_{+}, b\right)$ and $\left(\omega_{+}, b\right) \rightarrow\left(\omega_{-}, a\right)$.

If $P_{\omega}$ vanishes identically, then there exists an invariant circle of rotation number $\omega$. Therefore, if $\rho\left(\Gamma_{-}\right)<\omega<\rho\left(\Gamma_{+}\right)$, there exists $a \in \mathbb{R}$ such that $P_{\omega}(a)>0$. From Lemma 9.2, it follows that there exists $\varepsilon>0$ such that if $\omega-\varepsilon<\omega_{-}, \omega_{+}<\omega+\varepsilon$, then $\left(\omega_{-}, a\right) \rightarrow\left(\omega_{+}, a\right)$.

Lemma 9.4 follows from these remarks, together with Lemma 9.2 and the transitivity of the relation.

The conclusions of Lemma 9.4 still hold in many cases when $\omega_{-}$and $\omega_{+}$ are rational. To state the conditions under which they hold, we introduce the following notion: we say that a pair $(\omega, a)$ of real numbers is good if (a) $\omega$ is irrational and $P_{\omega}(a)>0$; if (b) $\omega=p / q$ is a rational number expressed in lowest terms, $P_{\omega}(a)>0$, and $q>2 \theta / P_{\omega}(a)$; or if (c) $\omega=p / q$ and $P_{p / q+}(a)>0$ or $P_{p / q-}(a)>0$.

Lemma 9.5. If $\left(\omega_{-}, a\right),\left(\omega_{+}, b\right)$ are good pairs and $\rho\left(\Gamma_{-}\right)<\omega_{-}, \omega_{+}<$ $\rho\left(\Gamma_{+}\right)$, then $\left(\omega_{-}, a\right) \rightarrow\left(\omega_{+}, b\right)$.

Proof. In the case that each of the pairs $\left(\omega_{-}, a\right),\left(\omega_{+}, b\right)$ satisfies conditions (a) or (b), the proof of Lemma 9.4 applies without change. Thus, it suffices to consider the case when $\left(\omega_{-}, a\right)$ or $\left(\omega_{+}, b\right)$ or both satisfies (c).

Consider, for example, the case when $\left(\omega_{-}, a\right)$ satisfies $(c)$, and $\left(\omega_{+}, b\right)$ satisfies (a) or (b). Suppose, for explicitness, that $\omega_{-}=p / q$ and $P_{p / q+}(a)>0$. Let $\omega$ be an irrational number slightly larger than $p / q+$. Then $P_{\omega}(a)>0$ by the estimate $\left|P_{p / q+}(a)-P_{\omega}(a)\right| \leq C \theta|\omega q-p|$ given in [17, Theorem 2.2]. Therefore, $(\omega, a)$ satisfies $(\mathrm{a})$, and we have $(\omega, a) \rightarrow\left(\omega_{+}, b\right)$. Thus, by 
transitivity of $\rightarrow$, it is enough to prove that $\left(\omega_{-}, a\right) \rightarrow(\omega, a)$. This may be done in the same way as Proposition 8.1 and Lemma 9.3 were proved. We leave the details to the reader.

The other cases may be treated similarly.

\section{Close APPROACH}

Lemma 10. Let $\omega$ be an irrational number. Let $a \in \mathbb{R}$ be such that $P_{\omega}(a)>0$. Let $y<x<z$ be minimal configurations of rotation number $\omega$. For each $i \in \mathbb{Z}$, let $a_{i}$ be the unique real number such that $a_{i}-a \in \mathbb{Z}$ and $x_{i} \in\left(a_{i}, a_{i}+\right.$ 1). There exists $K>0$ such that if $\mathscr{J}=\left(\ldots, J_{i}, \ldots\right)$ is a constraint with $J_{i}=\left[a_{i}, a_{i}+1\right]$ for $j_{0}-K \leq i \leq j_{1}+K$, then any $\mathscr{J}$-minimal configuration $\xi=\left(\ldots, \xi_{i}, \ldots\right)$ satisfies $y_{i}<\xi_{i}<z_{i}$, for $j_{0} \leq i \leq j_{1}$.

Proof. If $K$ is large enough, there exist $i_{0}, i_{1}$ that satisfy $j_{0}-K \leq i_{0} \leq j_{0}$ and $j_{1} \leq i_{1} \leq j_{1}+K$, such that $a_{i}+1<z_{i}$, for $i=i_{0}, i_{1}$. (In fact, if $\phi_{\omega}$ minimizes Percivals's Lagrangian (cf. §6) and $x_{i}=\phi_{\omega}(t+\omega i \pm), z_{i}=$ $\phi_{\omega}(s+\omega i \pm)$, it is enough to choose $K$ so that the projections of the intervals $(t, s),(t+\omega, s+\omega), \ldots,(t+(K-1) \omega, s+(K-1) \omega)$ on $\mathbb{R} / \mathbb{Z}$ cover $\mathbb{R} / \mathbb{Z}$. Since $\xi$ is a $\mathscr{J}$-configuration, we have $\xi_{i}<z_{i}$, for $i=i_{0}, i_{1}$. Therefore, since $\xi$ is $\mathscr{J}$-minimal and $z$ is minimal, we have $\xi_{i}<z_{i}$ for $i_{0} \leq i \leq i_{1}$, by the relative Aubry crossing lemma. The proof that $y_{i}<\xi_{i}$ is similar.

Corollary. Let $\omega, a, a_{i}$ be as above. Let $\varepsilon>0$. For any $i_{0} \in \mathbb{Z}$ there exist $K, K_{1}>0$ such that if $\mathcal{J}=\left(\ldots, J_{i}, \ldots\right)$ is a constraint with $J_{i}=\left[a_{i}, a_{i}+\right.$ 1] for $i_{0} \leq i \leq i_{1}$ (resp. $i_{-1} \leq i \leq i_{0}$ ), $\xi$ is a $\mathscr{J}$-minimal and $\mathscr{J}$-free configuration, and $\left(\ldots,\left(x_{i}, y_{i}\right), \ldots\right)$ and $\left(\ldots,\left(\xi_{i}, \eta_{i}\right), \ldots\right)$ are the $f$-orbits corresponding to $\left(\ldots, x_{i}, \ldots\right)$ and $\left(\ldots, \xi_{i}, \ldots\right)$, then

$$
\left|\xi_{i}-x_{i}\right|+\left|\eta_{i}-y_{i}\right|<\varepsilon,
$$

for $i_{0}+K \leq i \leq i_{1}-K_{1}$ (resp. $i_{-1}+K_{1} \leq i \leq i_{0}-K$ ).

This corollary provides close approach to a pre-assigned minimal orbit of rotation number satisfying $\rho\left(\Gamma_{-}\right)<\omega<\rho\left(\Gamma_{+}\right)$, provided that $\omega$ is irrational. This corollary is not true if the hypothesis that $\omega$ is irrational is dropped. However, there is an analogous result when $\omega$ is rational. This is provided by Lemma $\mathrm{f}$ in $\S 8$. It follows from Lemma $\mathrm{f}$ that for every $\varepsilon>0$, there exists $I$ such that if $i_{+}-i_{-} \geq I$, then $\xi_{i}-c<\varepsilon$. Thus, by taking $I$ large enough, we may arrange for the orbit corresponding to the configuration $\left(\ldots, \xi_{i}, \ldots\right)$ to pass as close as we like to the orbit corresponding to the configuration $(\ldots, c, \ldots)$.

\section{PROOF OF THE MAIN RESULTS AND FURTHER RESUlTS}

Proof of Theorem 4.1. First, suppose that $\rho\left(\Gamma_{-}\right)<\omega_{-}, \omega_{+}<\rho\left(\Gamma_{+}\right)$and both $\omega_{-}$and $\omega_{+}$are irrational. There exist $a, b>0$ such that $P_{\omega_{-}}(a), P_{\omega_{+}}(b)>$ 0 . According to Lemma 9.4 , we have $\left(\omega_{-}, a\right) \rightarrow\left(\omega_{+}, b\right)$. This relation asserts the existence of a constraint $\mathcal{J}^{*}=\left(\ldots, J_{i}^{*}, \ldots\right)$, if we take $i_{-2}=-\infty$ and 
$i_{2}=\infty$ in the definition (at the beginning of $\S 9$ ) of the relation $\rightarrow$. Let $x$ be a

$\mathscr{J}^{*}$-minimal configuration. According to the definition of the relation $\rightarrow, x$ is $\mathscr{J}^{*}$-free, and therefore (by Proposition 5.2) corresponds to an $f$-orbit. By the corollary of Lemma 10 , this orbit is $\alpha$-asymptotic to $M_{\bar{f}, \omega_{-}}$and $\omega$-asymptotic to $M_{\bar{f}, \omega_{+}}$.

In the case $\rho\left(\Gamma_{-}\right)<\omega_{-}, \omega_{+}<\rho\left(\Gamma_{+}\right)$and $\omega_{-}$or $\omega_{+}$(or both) is rational, we use a construction analogous to the construction of Proposition 8.1 to produce an orbit that is $\alpha$-asymptotic to $M_{\bar{f}, \omega_{-}}$and $\omega$-asymptotic to $M_{\bar{f}, \omega_{+}}$. For example, suppose that $\omega_{-}$is rational, say $\omega_{-}=p / q$ in lowest terms, and $\omega_{+}$is irrational. We use the same reduction as in $\S 8$, in particular using the variational principle $H$. Starting, for example, with a number $b$ such that $P_{p / q+}^{h}(b)=P_{0+}^{H}(b)>0$, we may construct a constraint $\mathscr{J}^{*}$ such that $\mathscr{J}^{*}$-minimal configurations are $\mathcal{J}^{*}$-free and the corresponding orbits are $\alpha$ asymptotic to $M_{\omega_{-}, \bar{f}}$ and $\omega$-asymptotic to $M_{\omega_{+}, \bar{f}}$, in the following way: First, we modify the construction of Proposition 8.1, letting

$$
\begin{array}{rlrl}
J_{i} & =[c, b], \quad \text { if } i<i_{+} & & \\
& =[b+\beta-1, b+\beta], & & \text { if } i_{+}+L_{+} \beta-L_{+} \leq i<i_{+}+L_{+} \beta \\
& & \text { and } 1 \leq \beta \leq l_{+}, \text {or } \beta=l_{+}+1 \text { and } i=i_{+}+L_{+} l_{+} .
\end{array}
$$

Here, $\left(c, c^{\prime}\right)$ is the complementary interval of $\pi\left(M_{F, 0}\right)=\pi\left(M_{f, p / q}\right)$ that contains $b, i_{+} \in \mathbb{Z}, L_{+} \geq L_{+}^{0}$, where $L_{+}^{0}$ is large enough (as large as in Proposition $8.1)$, and $l_{+} \geq 1$.

Then we modify the construction of Lemma 9.3, as follows: We set

$$
\widehat{J}_{i}^{+}=[b+\beta-1, b+\beta], \quad \text { if } i_{+}+L_{+} \beta-L_{+} \leq i \leq i_{+}+L_{+} \beta \text { and } \beta \in \mathbb{Z}
$$

and $\widehat{J}^{+}=\left(\ldots, \widehat{J}_{i}^{+}, \ldots\right)$. We let $\xi^{+}=\left(\ldots, \xi_{i}^{+}, \ldots\right)$ be the largest $\widehat{J}^{+}$. minimal configuration (for $H$ ). (Thus, $\xi^{+}$is a minimal configuration of rotation symbol $1 / L_{+}$.) We let $\xi^{*+}$ be the minimal configuration (for $h$ ) of rotation symbol $p / q+1 / q L_{+}$such that $\xi_{q i}^{*+}=\xi_{i}^{+}+p i$. We let $J_{\alpha}^{+}$be the closed interval $\left[a_{\alpha}^{*+}, a_{\alpha}^{*+}+1\right]$ defined by the conditions $a_{\alpha}^{*+}-b \in \mathbb{Z}$ and $\xi_{\alpha}^{*+} \in\left(a_{\alpha}^{*-}, a_{\alpha}^{*-}+1\right)$. We set

$$
\begin{array}{ll}
J_{\alpha}^{*}=J_{\alpha}^{*+}, & \text { for } q\left(i_{+}-1\right)<\alpha \leq q\left(i_{+}+L_{+} l_{+}+L_{+}-1\right), \\
J_{\alpha}^{*}=J_{i}+p i, & \text { for } \alpha=q i \text { and } i_{-} \leq i<i_{+}, \\
J_{\alpha}^{*}=\mathbb{R}, & \text { for all other } \alpha \leq q\left(i_{+}-1\right) .
\end{array}
$$

We choose an irrational number $\widehat{\omega}_{+}$slightly larger than $p / q+1 / q L_{+}$. Using Lemma 9.1, we extend $J_{\alpha}^{*}$ to be defined for $\alpha \leq i_{2}$, where $i_{2}>$ $q\left(i_{+}+L_{+} l_{+}^{\prime}+L_{+}-1\right)$, with the following properties: Let $J_{\alpha}^{+}=\left[a_{\alpha}^{+}, a_{\alpha}^{+}+1\right]$, where $a_{\alpha}^{+}-b \in \mathbb{Z}$ and $\hat{x}_{\alpha}^{+} \in\left(a_{\alpha}^{+}, a_{\alpha}^{+}+1\right)$, for some configuration $\hat{x}^{+}$of rotation number $\widehat{\omega}_{+}$. We require that $J_{\alpha}^{*}=J_{\alpha}^{+}+\lambda^{+}$for $i_{1} \leq \alpha \leq i_{2}$, where $\lambda^{+}$is a suitable integer. Here $q\left(i_{+}+L_{+} l_{+}^{\alpha}+L_{+}-1\right)<i_{1}<i_{2}-K_{1}$ and $i_{1}, K_{1}, i_{2}$ 
are chosen so that we can apply Lemma 9.1 to $\xi^{*+}$ and $\hat{x}^{+}$. By choosing the extension $\mathscr{J}^{*}=\left(\ldots, J_{i}^{*}, \ldots, J_{i(2)}^{*}\right)$ according to Lemma 9.1, we have that if $x=\left(\ldots, x_{i}, \ldots, x_{i(2)}\right)$ is a $\mathscr{J}^{*}$-minimal segment of a configuration, then $x_{\alpha} \in$ int $J_{\alpha}^{*}$ for $\alpha \leq i_{2}-K_{1}$. The proof that this is so requires a considerable amount of argument, but this was already explained in the proof of Lemma 9.3, and there seems to be no reason to repeat it again.

Let $a$ be such that $P_{\omega_{+}}(a)>0$. According to Lemma 9.4, we have $\left(\widehat{\omega}_{+}, b\right) \rightarrow$ $\left(\omega_{+}, a\right)$. Therefore, we may extend $\left(\ldots, J_{i}^{*}, \ldots, J_{i(2)}^{*}\right)$ to a constraint $\mathscr{J}^{*}=$ $\left(\ldots, J_{i}^{*}, \ldots\right)$ such that every $\mathscr{J}^{*}$-minimal configuration is $\mathscr{J}^{*}$-free and such that the corresponding orbit is $\omega$-asymptotic to $M_{\bar{f}, \omega_{+}}$, by the corollary of Lemma 10.

The cases when $\omega_{+}$is rational and $\omega_{-}$is irrational, or both are rational, may be treated similarly.

So far, we have proved Theorem 4.1 in the case that $\rho\left(\Gamma_{-}\right)<\omega_{-}, \omega_{+}<$ $\rho\left(\Gamma_{+}\right)$. Now we consider the case when $\omega_{-}=\rho\left(\Gamma_{ \pm}\right)$or $\omega_{+}=\rho\left(\Gamma_{ \pm}\right)$, or both. For example, suppose that $\omega_{-}=\rho\left(\Gamma_{-}\right)$. We choose a sequence $\left(\omega_{j}\right)_{j \leq 0}$ of irrational numbers such that $\omega_{j} \downarrow \omega_{-}$, as $j \geq-\infty$ and a sequence of numbers $a_{j}$ such that $P_{\omega(j)}\left(a_{j}\right)>0$. According to Lemma 9.4, $\left(\omega_{j}, a_{j}\right) \geq$ $\left(\omega_{j+1}, a_{j+1}\right)$, for $j+1 \leq 0$. This means that there exist integers $K_{j}, \widetilde{K}_{j}$ for $j \leq 0$ such that the following hold: If $x^{j}=\left(\ldots, x_{i}^{j}, \ldots\right)$, for $j \leq 0$, are minimal configurations with rotation numbers $\rho\left(x^{j}\right)=\omega_{j}$, constraints $\mathscr{J}^{j}=$ $\left(\ldots, J_{i}^{j}, \ldots\right)$ are defined by $J_{i}^{j}=\left[a_{i}^{j}, a_{i}^{j}+1\right]$, where $a_{i}^{j}-a_{i} \in \mathbb{Z}$ and $x_{i}^{j} \in$ $\left(a_{i}^{j}, a_{i}^{j}+1\right)$, and $i_{j}, \widetilde{i}_{j}$ are integers satisfying

$$
i_{j}+K_{j}<\widetilde{i}_{j}<\widetilde{i}_{j}+\widetilde{K}_{j}<i_{j+1}
$$

and $\widetilde{i}_{0}=0$, then there exist integers $l_{j}, j \leq 0$, and a sequence $\left(\ldots, J_{j}^{*}, \ldots\right.$, $J_{0}^{*}$ ) of closed intervals such that

$$
J_{i}^{*}=J_{i}^{j}+l_{j}, \quad \text { for } i_{j} \leq i \leq \widetilde{i}_{j}
$$

and such that if $x=\left(\ldots, x_{i}, \ldots, x_{0}\right)$ is any segment of a configuration satisfying $x_{i} \in J_{i}^{*}$, for $i \leq 0$, and $x$ is $\mathcal{J}^{*}$-minimal, then $x_{i} \in \operatorname{int} J_{i}^{*}$, for $i \leq-K_{0}$.

Moreover, the proof of Lemma 9.4 shows that the intervals $J_{i}^{*}=\left[a_{i}^{*}, a_{i}^{*}+1\right]$ can be chosen so that

$$
\lim _{i \rightarrow-\infty} a_{i}^{*} / i=\omega_{-} .
$$

In the case that $\rho\left(\Gamma_{-}\right)<\omega_{+}<\rho\left(\Gamma_{+}\right)$, the arguments that we have given previously show that it is possible to extend $\mathscr{J}^{*}$ to a constraint $\widetilde{J}^{*}=\left(\ldots, \widetilde{J}_{i}^{*}\right.$, ...) such that every $\widetilde{J}^{*}$-minimal configuration $x$ is $\widetilde{J}^{*}$-free and has the property that the corresponding $\bar{f}$-orbit is $\omega$-asymptotic to $M_{\bar{f}, \omega_{+}}$. 
This $\bar{f}$-orbit will be $\alpha$-asymptotic to $M_{\bar{f}, \omega_{-}}$, provided that $\rho\left(\Gamma_{-}\right)=\omega_{-}$is irrational, since $\lim _{i \rightarrow-\infty} a_{i}^{*} / i=\omega_{-}$. Thus, we have proved Theorem 9.1 in the following two cases: when $\rho\left(\Gamma_{-}\right)<\omega_{-}, \omega_{+}<\rho\left(\Gamma_{+}\right)$and when $\rho\left(\Gamma_{-}\right)=$ $\omega_{-}<\omega_{+}<\rho\left(\Gamma_{+}\right)$and $\omega_{-}$is irrational. The remaining cases may be proved in a similar fashion.

Now, some comments on Theorem 4.1 and its proof. The hypothesis that if $\omega_{-}\left(\right.$resp. $\left.\omega_{+}\right)=\rho\left(\Gamma_{-}\right)\left(\rho\left(\Gamma_{+}\right)\right)$, then $\omega_{-}$(resp. $\left.\omega_{+}\right)$is irrational may appear mysterious. Actually, when this condition fails to hold, there is a similar statement, only it is a bit more complicated. Consider, for example, the case when $\omega_{-}=\rho\left(\Gamma_{-}\right)$, and this number is rational. Since $\Gamma_{-}$is an invariant curve, it consists of minimal orbits. One possibility is that all these orbits have rotation symbol $\omega_{-}$or $\omega_{-}+$. In this case, the construction above gives an orbit that is $\alpha$-asymptotic to $\Gamma_{-}$, but not to $M_{\bar{f}, \omega}$, which is in general only a subset of $\Gamma_{-}$. In fact, in this case, if $M_{\bar{f}, \omega_{-}} \neq \Gamma_{-}$it is impossible to construct an orbit that is $\alpha$-asymptotic to $M_{\bar{f}, \omega_{-}}$.

The other possibility is that some of the orbits in $\Gamma_{-}$have rotation symbol $\omega_{-}-$. In this case $P_{\omega_{-}+}(a)>0$, for some $a \in \mathbb{R}$, and the construction used in the proof above for the case $\rho\left(\Gamma_{-}\right)<\omega_{-}<\rho\left(\Gamma_{+}\right)$and $\omega_{-}$is irrational still works. Consequently, the conclusion of Theorem 4.1 still holds in this case as long as $\omega_{+}$satisfies the hypothesis.

The cases when $\omega_{-}=\rho\left(\Gamma_{+}\right)$and $\omega_{+}$is rational, or two of these conditions hold, may obviously be treated similarly.

Theorem 4.1 and its proof raise the following question: Given minimal $\bar{f}$ orbits $\mathscr{O}_{-}$and $\mathscr{O}_{+}$in a Birkhoff region of instability, is it possible to construct an $\bar{f}$-orbit $\mathscr{O}$ that is $\alpha$-asymptotic to $\mathscr{O}_{-}$and $\omega$-asymptotic to $\mathscr{O}_{+}$? If $\rho\left(\Gamma_{-}\right)<$ $\rho\left(\mathscr{O}_{-}\right), \rho\left(\mathscr{O}_{+}\right)<\rho\left(\Gamma_{+}\right)$and both $\rho\left(\mathscr{O}_{-}\right)$and $\rho\left(\mathscr{O}_{+}\right)$are irrational, then there exists such an $\bar{f}$-orbit. For, according to Lemma 9.4 , we have $\left(\omega_{-}, a\right) \rightarrow$ $\left(\omega_{+}, b\right)$. If we take $x_{-}$and $x_{+}$in the definition (at the beginning of $\left.\S 9\right)$ of the relation to be the relation $\rightarrow$ to be the first component of $\mathscr{O}_{-}$and $\mathscr{O}_{+}$, resp., then the corollary of Lemma 10 gives the required conclusion.

On the other hand, if one of $\rho\left(\mathscr{O}_{-}\right)$and $\rho\left(\mathscr{O}_{+}\right)$is one of $\rho\left(\Gamma_{-}\right)$and $\rho\left(\Gamma_{+}\right)$ and that number is irrational, then our method is insufficient for this conclusion and we do not know the answer.

As usual, the case when one or both of $\rho\left(\mathscr{O}_{-}\right)$or $\rho\left(\mathscr{O}_{+}\right)$is rational is more complicated. Suppose, for example, that $\rho\left(\mathscr{O}_{-}\right)$is a rational number $p / q$ in lowest terms, that $\rho\left(\mathscr{O}_{+}\right)$is irrational, and that $\rho\left(\Gamma_{-}\right)<\rho\left(\mathscr{O}_{-}\right), \rho\left(\mathscr{O}_{+}\right)<\rho\left(\Gamma_{+}\right)$. If $\mathscr{O}_{-}$is not periodic, then it is $\alpha$-asymptotic to a periodic orbit and the question of whether there exists an orbit that is $\alpha$-asymptotic to $\mathscr{O}_{-}$is equivalent to the question of whether there exists an orbit that is $\alpha$-asymptotic to that periodic orbit.

Thus, we may as well assume that $\mathscr{O}_{-}$is periodic. In the case that $\pi\left(M_{\bar{f}, p / q}\right)$ is finite, and neither $\pi\left(M_{\bar{f}, p / q_{+}}\right)$or $\pi\left(M_{\bar{f}, p / q_{-}}\right)$contains interior points, there 
exists an orbit $\mathscr{O}$ that is $\alpha$-asymptotic to $\mathscr{O}_{-}$and $\omega$-asymptotic to $\mathscr{O}_{+}$. This may be proved by a slight modification of the argument used in the proof of Theorem 4.1 for the case $\omega_{-}$is rational, $\omega_{+}$is irrational, and $\rho\left(\Gamma_{-}\right)<\omega_{-}$, $\omega_{+}<\rho\left(\Gamma_{+}\right)$. It is enough to choose the appropriate complementary interval $\left(c, c^{\prime}\right)$ of $\pi\left(M_{\bar{f}, p / q}\right)$ and then argue as before. If $\mathscr{O}_{-}=\left(\ldots,\left(x_{i}, t_{i}\right), \ldots\right)$, it is enough to take $c=x_{0}$. Note that the hypothesis that $\pi\left(M_{\bar{f}, p / q+}\right)$ does not contain interior points implies that there exists $b \in\left(c, c^{\prime}\right)$ such that $P_{p / q+}(b)>$ 0 . If we choose $\left(c, c^{\prime}\right)$ and $b$ this way, then the constraint $\mathcal{J}^{*}$ that we constructed in the proof of Theorem 4.1 (for the case $\omega_{-}$is rational, $\omega_{+}$is irrational and $\left.\rho\left(\Gamma_{-}\right)<\omega_{-}, \omega_{+}<\rho\left(\Gamma_{+}\right)\right)$has the property (as before) that $\mathscr{J}^{*}$-minimal configurations are $\mathcal{J}^{*}$-free and the additional property that the corresponding orbits are $\alpha$-asymptotic to $\mathscr{O}_{-}$. As already explained (in the case that both $\omega_{-}$and $\omega_{+}$are irrational) it is also possible to construct $\mathcal{J}^{*}$ minimal configurations are $\omega$-asymptotic to $\mathscr{O}_{+}$.

The other cases (when $\rho\left(\mathscr{O}_{+}\right)$or both are rational) may be treated similarly.

Proof of Theorem 4.2. First, we suppose that all the $\omega_{i}$ are irrational and satisfy $\rho\left(\Gamma_{-}\right)<\omega_{i}<\rho\left(\Gamma_{+}\right)$. For each $i$, we choose $a_{i}$ such that $P_{\omega(i)}\left(a_{i}\right)>$ 0 . According to Lemma 9.4, $\left(\omega_{j}, a_{j}\right) \rightarrow\left(\omega_{j+1}, a_{j+1}\right)$, for all $j \in \mathbb{Z}$. This means that there exist integers $K_{j}, \widetilde{K}_{j}$ such that the following hold: If $x^{j}=$ $\left(\ldots, x_{i}^{j}, \ldots\right)$ are minimal configurations with $\rho\left(x^{j}\right)=\omega_{j}$, constraints $\mathscr{J}^{j}=$ $\left(\ldots, J_{i}^{j}, \ldots\right)$ are defined by $J_{i}^{j}=\left[a_{i}^{j}, a_{i}^{j}+1\right]$, where $a_{i}^{j}-a_{i} \in \mathbb{Z}$ and $x_{i}^{j} \in$ $\left(a_{i}^{j}, a_{i}^{j}+1\right)$, and $i_{j}, \widetilde{i}_{j}$ are integers satisfying

$$
i_{j}+K_{j}<\tilde{i}_{j}<\widetilde{i}_{j}+\widetilde{K}_{j}<i_{j+1},
$$

then there exist integers $l_{j}, j \leq 0$, and a sequence $\left(\ldots, J_{j}^{*}, \ldots\right)$ of closed intervals such that

$$
J_{i}^{*}=J_{i}^{j}+l_{j}, \quad \text { for } i_{j} \leq i \leq \widetilde{i}_{j}
$$

and such that if $x=\left(\ldots, x_{i}, \ldots\right)$ is any segment of a configuration satisfying $x_{i} \in J_{i}^{*}$ and $x$ is $\mathscr{J}^{*}$-minimal, then $x_{i} \in \operatorname{int} J_{i}^{*}$, for all $i \in \mathbb{Z}$.

Consider one such $\mathscr{J}^{*}$-minimal configuration $\left(\ldots, x_{i}, \ldots\right)$ and let $\mathscr{O}=$ $\left(\ldots,\left(x_{i}, y_{i}\right), \ldots\right)$ denote the corresponding orbit. From Lemma 10, it follows that $\mathscr{O}$ approaches within $\varepsilon_{j}$ of $M_{\bar{f}, \omega(j)}$, provided that $K_{j}$ is chosen large enough. Thus, Theorem 4.2 is proved in this case.

In fact, if we let $\mu_{\bar{f}, \omega(j)}$ be the unique minimal measure of rotation number $\omega_{j}$, this construction yields an orbit $\mathscr{O}$ that approaches within $\varepsilon_{j}$ of $\mu_{\bar{f}, \omega(j)}$. To see this it is enough to choose $x^{j}$ so that the corresponding orbit is recurrent (and therefore lies in $\left.\mu_{\bar{f}, \omega(j)}\right)$.

The remaining cases may be reduced to this case. For example, if one of the $\omega_{j}$ 's is rational, we may replace it by an $\omega_{j}^{\prime}$ that is irrational and as close as we like to $\omega_{j}$. There are points on $\mu_{\bar{f}, \omega(j)^{\prime}}$ that are close to $M_{\bar{f}, \omega(j)}$; we may 
arrange for this distance to be as small as we like by taking $\omega_{j}^{\prime}$ close enough to $\omega_{j}$. Furthermore, any orbit in $\mu_{\bar{f}, \omega(j)^{\prime}}$ passes close to $M_{\bar{f}, \omega(j)}$, since orbits in the former are dense in the former. Therefore an orbit that passes close enough to $\mu_{\bar{f}, \omega(j)^{\prime}}$ will pass close to $M_{\bar{f}, \omega(j)}$, and the problem of constructing orbits that pass close to $M_{\bar{f}, \omega(j)}$ is reduced to constructing orbits that pass close to $\mu_{\bar{f}, \omega(j)^{\prime}}$, which has already been done in the previous construction.

The case when $\omega_{j}=\rho\left(\Gamma_{-}\right)$or $\rho\left(\Gamma_{+}\right)$may also be treated by using an irrational $\omega_{j}^{\prime}$ satisfying $\rho\left(\Gamma_{-}\right)<\omega_{j}^{\prime}<\rho\left(\Gamma_{+}\right)$, which approximates $\omega_{j}$, and reducing to the case already treated.

\section{APPENDIX. BIRKHOFF'S THEOREM}

The theorem we mean is the following:

Theorem. Let $U$ be an open subset of $(\mathbb{R} / \mathbb{Z}) \times \mathbb{R}$ and suppose that there exist $A<B \in \mathbb{R}$ such that $(\mathbb{R} / \mathbb{Z}) \times(-\infty, A] \subset U$ and $\mathbb{R} / \mathbb{Z} \times[B, \infty) \cap U=\varnothing$. Suppose that $U$ is homeomorphic to an annulus and invariant under $\bar{f}$. Then the frontier of $U$ is the graph of a Lipschitz function $u: \mathbb{R} / \mathbb{Z} \rightarrow \mathbb{R}$.

Something very similar to this was stated by Birkhoff at least three times [4, pp. 195-196, 422-426, 447], the first two times with a proof that, however, appears to us not to be complete. More recently, complete proofs have been given $[6,7]$ for twist diffeomorphism, i.e., for $\bar{f} \in \mathscr{T}^{1}$. A very interesting generalization, to more degrees of freedom, is contained in [8].

It is not clear that the methods of [6] or [7] work for $f \in \mathscr{P}^{1}$. In this appendix, we give a proof of Birkhoff's theorem for $f \in \mathscr{P}^{1}$. This proof follows the method of Birkhoff.

We consider a $C^{1}$ curve $\gamma:(-\infty, 0] \rightarrow \mathbb{R} / \mathbb{Z} \times \mathbb{R}$ such that $\gamma$ is a $C^{1}$ embedding (i.e., it is injective, and $\gamma^{\prime}$ vanishes nowhere) and $\gamma_{2}(t)$ (the second coordinate of $\gamma(t)$ ) goes to $-\infty$ as $t$ goes to $-\infty$. We define the tilt $\tau_{\gamma}$ of $\gamma$, as follows: $\tau_{\gamma}(t) \equiv \operatorname{arccot} \gamma_{1}^{\prime}(t) / \gamma_{2}^{\prime}(t)(\bmod 2 \pi)$, and if $\gamma_{2}(s) \leq \gamma_{2}\left(t_{0}\right)$ for $s \leq t_{0}$, then $\left|\tau_{\gamma}\left(t_{0}\right)\right| \leq \pi / 2$. It is easily seen that there is exactly one continuous real-valued function $\tau_{\gamma}$ on $(-\infty, 0]$ that satisfies these two conditions.

As a first step towards proving this, consider the case when $\gamma_{1}^{\prime}(t)=0$ for $t$ sufficiently small. In this case, we define $\tau_{\gamma}(t)=0$ for $t$ sufficiently small. To prove that $\left|\tau_{\gamma}\left(t_{0}\right)\right| \leq \pi / 2$ when $\gamma_{2}(s) \leq \gamma_{2}\left(t_{0}\right)$ for $s \leq t_{0}$, we consider $t_{1}<t_{0}$ such that $\gamma_{1}^{\prime}(s)=0$ if $s \leq t_{1}$ and $\gamma_{2}\left(t_{1}\right)<\gamma_{2}(s)<\gamma_{2}\left(t_{0}\right)$ if $t_{1} \leq s \leq t_{0}$. Let $A$ be the annulus $\mathbb{R} / \mathbb{Z} \cap\left[t_{1}, t_{0}\right]$ and let $\Gamma=\gamma\left[t_{1}, t_{0}\right] \subset A$. A standard result in differential topology says that there is a $C^{1}$ isotopy of $\Gamma$ to a straight line. The required result, i.e., $\left|\tau_{y}\left(t_{0}\right)\right| \leq \pi / 2$, follows immediately.

We may reduce the general situation to this case, as follows: It is clear that there is a continuous function $\tau_{\gamma}$ on $(-\infty, 0]$ such that

$$
\tau_{\gamma}(t) \equiv \operatorname{arccot} \gamma_{1}^{\prime}(t) / \gamma_{2}^{\prime}(t) \quad(\bmod 2 \pi) .
$$


Such a function is defined up to addition of $2 \pi n$, where $n \in \mathbb{Z}$. To see that it is possible to choose $\tau_{\gamma}$ with the required property, it is enough to show that if $t_{0}<t_{1}$ satisfy the conditions $s<t_{0}$, implies $\gamma_{2}(s)<\gamma_{2}\left(t_{0}\right), s<t$, implies $\gamma_{2}(s)<\gamma_{2}\left(t_{1}\right)$, and $\gamma_{2}^{\prime}\left(t_{0}\right), \gamma_{2}^{\prime}\left(t_{1}\right)>0$, then $\left|\tau_{\gamma}\left(t_{0}\right)-\tau_{\gamma}\left(t_{1}\right)\right|<\pi$. For this purpose, we may (using standard results in differential topology) isotope $\gamma$ to a curve for which $\gamma_{1}^{\prime}(t)=0$ for $t$ sufficiently small, without altering $\gamma\left|\left[t_{0}, t_{1}\right]\right|$. For the new $\gamma$, we have already defined $\tau_{\gamma}$ with the required property. We may use the isotopy to define $\tau_{\gamma}$ for the new $\gamma$. Since the isotopy does not alter $\gamma\left|\left[t_{0}, t_{1}\right]\right|$, it does not alter $\tau_{\gamma}\left|\left[t_{0}, t_{1}\right]\right|$ either, so the original $\tau_{\gamma}$ has the required property.

We will say that $\gamma$ is positively tilted if $\tau_{\gamma}$ is everywhere positive and negatively tilted if $\tau_{\gamma}$ is everywhere negative.

From the fact that $\bar{f} \in \mathscr{P}^{1}$, it is easy to prove that if $\gamma$ is positively tilted, then $\bar{f} \circ \gamma$ is positively tilted, and if $\gamma$ is negatively tilted, then $\bar{f}^{-1} \circ \gamma$ is negatively tilted.

A point $x \in U$ will be said to be positively (resp. negatively) accessible if there is a positively (resp. negatively) tilted $C^{1}$ curve $\gamma:(-\infty, 0] \rightarrow U$ such that $\gamma(0)=x$. We let $U_{+}$(resp. $U_{-}$) denote the set of positively (resp. negatively) accessible points in $U$. By the result of the previous paragraph, and the assumption that $U$ is $\bar{f}$-invariant, we have $\bar{f} U_{+} \subset U_{+}$and $\bar{f}^{-1} U_{-} \subset U$.

We say that a point $x=\left(x_{1}, x_{2}\right) \in U$ is vertically accessible if all points $\left(x_{1}, y\right)$ with $y \leq x_{2}$ are in $U$. We let $U_{0}$ denote the set of all vertically accessible points. At the end of this appendix, we sketch a proof that $U_{0}=$ $U_{-} \cap U_{+}$. Essentially the same result is stated in [9, Proposition 1.2] with a reference to [10] and [4, pp. 422-426] for the proof. However, we were unable to find a proof in either reference, although similar things are proved in both references, and presumably this proof could be worked out by anyone who studied those references.

From $U_{0}=U_{-} \cap U_{+}$, it follows that if $U_{0} \neq U$, we have $U_{+} \neq U$ or $U_{-} \neq U$. If $U_{+} \neq U$, it is easily seen that $\bar{f} U_{+}$is a proper subset of $U_{+}$. But this contradicts the assumption that $\bar{f}$ is exact area-preserving. Similarly, if $U_{-} \neq U$, it is easily seen that $\bar{f}^{-1} U_{-}$is a proper subset of $U_{-}$, and again this contradicts the fact that $\bar{f}$ is exact area-preserving.

This contradiction shows that $U_{0}=U$.

Let $h_{\varepsilon}(x, y)=(x, y+\varepsilon x)$, for $(x, y) \in \mathbb{R}^{2}$ and let $\bar{h}_{\varepsilon}$ denote the induced diffeomorphism of $(\mathbb{R} / \mathbb{Z}) \times \mathbb{R}$. For $\varepsilon>0$ sufficiently small, $\bar{h}_{\varepsilon} \circ \bar{f} \circ \bar{h}_{\varepsilon}^{-1}$ and $\bar{h}_{\varepsilon}^{-1} \circ \bar{f} \circ \bar{h}_{\varepsilon} \in \mathscr{P}$. Thus, we may apply the result just obtained to these diffeomorphisms, replacing $U$ with $\bar{h}_{\varepsilon}(U)$ in the first case and with $\bar{h}_{\varepsilon}^{-1}(U)$ in the second case.

From the result just obtained, we see that $\bar{h}_{\varepsilon}(U)_{0}=\bar{h}_{\varepsilon}(U)$ and $\bar{h}_{\varepsilon}^{-1}(U)_{0}=$ $\bar{h}_{\varepsilon}^{-1}(U)$, where the subscript 0 denotes the set of vertically accessible points, in both cases. 
Since this is true for all sufficiently small $\varepsilon>0$, it follows that the frontier of $U$ is the graph of a Lipschitz function.

The above argument is due to Birkhoff. However, Birkhoff takes it as obvious that if $U_{0} \neq U$ then $U_{-} \neq U$ or $U_{+} \neq U$. Since we had difficulty on this point, we present a proof below.

Proof that $U_{0}=U_{-} \cap U_{+}$. Obviously, $U_{0} \subset U_{-} \cap U_{+}$. To show the opposite inclusion, we consider a point $P$ in $U \backslash U_{0}$ and show that it is not in $U_{-} \cap U_{+}$. We let $W$ denote the connected component of $U \backslash U_{0}$ that contains $P$. We let $L$ denote the frontier of $W$ in $V$. From the definition of $U_{0}$, it follows that $L$ is a union of vertical line segments.

In fact, $L$ is a single vertical line segment, i.e., it has only one connected component. For, if $L_{0}$ and $L_{1}$ were two connected components in $L$, we would obtain a contradiction, as follows. We could choose a simple arc $\Lambda_{0}$ joining a point $P_{0}$ of $L_{0}$ to a point $P_{1}$ of $L_{1}$ and lying in $W$ (except for its endpoints). Likewise, we could choose a simple arc $\Lambda_{1}$ joining $P_{0}$ and $P_{1}$ and lying in $V_{0}$ (except for its endpoints). Then $\Lambda_{0} \cup \Lambda_{1}$ would be a simple closed curve in $(\mathbb{R} / \mathbb{Z}) \times \mathbb{R}$ and so separate $(\mathbb{R} / \mathbb{Z}) \times \mathbb{R}$ into two regions, one of which, $R$, would be in $U$. However, the endpoints of $L_{0}$ are in the complement of $U$ (by the definition of $L$ ) and one would be in $R$ (since $\Lambda_{0} \cup \Lambda_{1}$ crosses $L_{0}$ exactly once), a contradiction. This contradiction shows that $L$ is a single vertical line segment.

Obviously, points near one side of $L$ are in $U_{0}$, but not points on the other side. Consider, for example, the case when points near the right side of $L$ are in $U_{0}$.

We assert that $P \notin U_{+}$, in this case. Suppose otherwise. Then there is a positively tilted arc $\gamma:(-\infty, 0] \rightarrow(\mathbb{R} / \mathbb{Z}) \times \mathbb{R}$ in $U$ such that $\gamma(0)=P$.

Now we consider the picture in the universal cover. We let $\widetilde{P}$ denote a lift of $P$ in the universal cover. We let $\tilde{\gamma}$ be a lift of $\gamma$ to the universal cover such that $\widetilde{\gamma}(0)=\widetilde{P}$. We let $\widetilde{W}$ be the component of the inverse image of $W$ in the universal cover such that $\widetilde{P} \in \widetilde{W}$. We let $\widetilde{L}$ denote the frontier of $\widetilde{W}$ in the inverse image of $U$. Thus, $\widetilde{L}$ is a lift of $L$ to the universal cover, and is a single vertical line segment. We let $\widetilde{L}^{*}$ denote the set of all points on $\widetilde{L}$ or directly below $\widetilde{L}$ (so $\widetilde{L}^{*}$ is a vertical ray in $\mathbb{R}^{2}$ ). We let $\widetilde{L}^{* *}$ denote the vertical line that contains $\widetilde{L}^{*}$.

Let $\widetilde{Q}_{0}=\widetilde{\gamma}\left(t_{0}\right)$ be the highest point on $\widetilde{L}^{* *}$ where $\widetilde{\gamma}(-\infty, 0]$ intersects $\widetilde{L}^{* *}$. The discussion in $[9,4.3]$ shows that $\widetilde{\gamma}\left(-\infty, t_{0}\right)$ is entirely to the left of $\widetilde{L}^{* *}$, for otherwise $\tilde{\gamma}$ would not have positive tilt. (Note that $\widetilde{\gamma}(-\infty, 0]$ intersects $\widetilde{L}$, since $\widetilde{P}=\widetilde{\gamma}(0) \in \widetilde{W}, \widetilde{L}$ is the frontier of $\widetilde{W}$ in the inverse image of $U$, and $\gamma(t) \in U$, for $t$ sufficiently small.) Thus, there is a smallest $t_{1}$ such that $\tilde{\gamma}\left(t_{1}\right) \in \widetilde{L}^{*}$ and $t_{1} \geq t_{0}$.

Now we consider two cases depending on whether $\tilde{\gamma}\left(t_{1}-\delta\right)$ is to the left or to the right of $\widetilde{L}^{*}$ for small $\delta>0$. In the case that $\tilde{\gamma}\left(t_{1}-\delta\right)$ is to the left, the 
argument of $[9,4.3]$ shows that $\widetilde{Q}_{1}=\widetilde{\gamma}\left(t_{1}\right)$ is the highest point on $\widetilde{L}^{*}$ where $\tilde{\gamma}(-\infty, 0]$ intersects $\widetilde{L}^{*}$, for otherwise $\tilde{\gamma}$ would not have positive tilt. Since $\widetilde{\gamma}(-\infty, 0]$ intersects $\widetilde{L}$ and all points on $\widetilde{L}^{*}$ are on or below $\widetilde{L}$, it follows that $\widetilde{Q}_{1} \in \widetilde{L}$. But then $\widetilde{\gamma}\left(t_{1}-\delta\right) \in \widetilde{W}$, since points in the inverse image of $U$ to the left of $\widetilde{L}$ are in $\widetilde{W}$. However, then $\widetilde{\gamma}(t)$ crosses $\widetilde{L}$ for some $t<t_{1}-\delta$, contradicting the definition of $t_{1}$ (as the smallest $t$ such that $\tilde{\gamma}(t) \in \widetilde{L}^{*}$ ). This contradiction shows that $\widetilde{\gamma}\left(t_{1}-\delta\right)$ is not to the left of $\widetilde{L}^{*}$.

In the case that $\tilde{\gamma}\left(t_{1}-\delta\right)$ is to the right of $\widetilde{L}^{*}$, we let $\widetilde{L}^{\prime}$ be the vertical ray descending from $\tilde{\gamma}\left(t_{1}-\delta\right)$. Since $\gamma\left(t_{1}-\delta\right) \in U_{0}$, the projection $L^{\prime}$ of $\widetilde{L}^{\prime}$ on $(\mathbb{R} / \mathbb{Z}) \times \mathbb{R}$ is in $U$. Thus, $\Lambda=\tilde{\gamma}\left(-\infty, t_{1}-\delta\right] \cup \widetilde{L}^{\prime}$ is in the inverse image of $U$. It is a simple closed curve and separates $\mathbb{R}^{2}$ into two parts, one containing $\mathbb{R} \times[B, \infty)$, for $B$ large enough, the other containing $\widetilde{L}$ and being contained in the inverse image of $U$. However, since the endpoints of $\widetilde{L}$ are not in the inverse image of $U$, this is a contradiction.

This contradiction shows that $P \notin U_{+}$when points near the right side of $L$ are in $U_{0}$. A similar argument shows that $P \notin U_{-}$when points near the left side of $L$ are in $U_{0}$. Thus, in either case, $P \notin U_{-} \cap U_{+}$, as asserted.

\section{ACKNOWLEDGMENTS}

I would like to thank ETH for its hospitality. I did much of the work leading to the results in this paper at ETH as well as the work of writing up the proofs, not only in this paper, but also in the papers [16-18], which contain results needed for this paper. I announced versions of Theorems 4.1 and 4.2 in lectures I gave at ETH and Oberwolfach in 1985. I would especially like to thank Jürgen Moser, Edie Zehnder, Eugene Trubowitz, and others at ETH who have expressed interest in this work. I would like to thank Michael Herman for inviting me to talk on this and related results several times in his seminar. I would like to thank Charles Fefferman for listening to an oral exposition of these results. Without the interest and encouragement of these mathematicians, I would never have had the energy to finish this paper. I would like to thank Giovanni Forni for pointing out an error in the proof of Lemma $g$ in $\S 8$ of the preprint version of this article. Accordingly, I have changed the statement of Lemma $g$ and the proof of Lemmas $h$ and $i$.

I would like to thank Rahel Boller for the care she took typing this manuscript.

I finished this paper while visiting ETH on a Guggenheim Fellowship. I would like to thank the Guggenheim Foundation for its support.

\section{REFERENCES}

1. S. Aubry and P. Y. Le Daeron, The discrete Frenkel-Kontorova model and the devil's staircase, Phys. D 7 (1983), 240-258.

2. S. Aubry, P. Y. Le Daeron, and G. André, Classical ground states of a one-dimensional model for incommensurate structures, preprint, 1982. 
3. V. Bangert, Mather sets for twist maps and geodesics on tori, Dynamics Reported 1 (1988), $1-54$.

4. G. D. Birkhoff, Collected mathematical papers, vol. II, Amer. Math. Soc., Providence, RI, 1950.

5. C. Carathéodory, Variationsrechnung und partielle Differentialgleichung erster Ordnung, Teubner, Leipzig, Berlin, 1935.

6. A. Fathi, Appendix to Chapter I of [7].

7. M. R. Herman, Sur les courbes invariantes par les difféomorphismes de l'anneau, vol. 1, Astérisque, nos. 103-104, Soc. Math. France, Paris, 1983.

8. __ Inequalités a priori pour des tores lagrangiens invariants par des difféomorphismes symplectiques, preprint, September 1989.

9. P. Le Calvez, Propriétés générales des applications déviant la verticale, Bull. Soc. Math. France 117 (1989), 69-102.

10. __ Propriétés des attracteurs de Birkhoff, Ergodic Theory Dynamical Systems 8 (1988), 241-310.

11. R. S. MacKay, J. D. Meiss, and I. C. Percival, Transport in Hamiltonian systems, Phys. D 13 (1984), 55-81.

12. J. N. Mather, Existence of quasi-periodic orbits for twist homeomorphisms of the annulus, Topology 21 (1982), 457-467.

13. _ A criterion for the non-existence of invariant circles, Inst. Hautes Études Sci. Publ. Math. 63 (1986), 153-204.

14. __ Letter to R. MacKay, Feb. 21, 1984.

15. __, More Denjoy minimal sets for area preserving diffeomorphisms, Comment. Math. Helv. 60 (1985), 508-557.

16. __ Modulus of continuity for Peierls's barrier, Periodic Solutions of Hamiltonian Systems and Related Topics (P. H. Rabinowitz et al., eds.), NATO ASI Series C, vol. 209, Reidel, Dordrecht, 1987, pp. 177-202.

17. _ Destruction of invariant circles, Ergodic Theory Dynamical Systems 8 (1988), 199214.

18. __ Minimal measures, Comment. Math. Helv. 64 (1989), 375-394.

19. I. C. Percival, Variational principles for invariant tori and cantori, Sympos. on Nonlinear Dynamcis and Beam-Beam Interactions (M. Month and J. C. Herrara, eds.), American Inst. of Phys. Conf. Proc., no. 57, 1980, pp. 302-310.

20. __, A variational principle for invariant tori of fixed frequency, J. Phys. A 12 (1979), L57-L60.

21. __ Definite paths and upper bounds on regular regions of velocity phase space, Phys. D 6 (1982/83), 249-259.

22. E. C. Titchmarsh, The theory of functions, Clarendon Press, Oxford, 1932.

Department of Mathematics, Princeton University, Princeton, New Jersey 08544 